\title{
Simulação Numérica Bidimensional da Interação Fluido-Estrutura através do Método Físico Virtual
}

\author{
Santos Alberto Enriquez Remigio
}

TESE APRESENTADA

$\mathrm{AO}$

INSTITUTO DE MATEMÁTICA E ESTATÍSTICA

DA

UNIVERSIDADE DE SÃO PAULO

PARA OBTENÇÃO DO GRAU

$\mathrm{DE}$

DOUTOR EM MATEMÁTICA APLICADA

Área de Concentração:

Matemática Aplicada

Orientador: Prof. Dr. Alexandre Megiorin Roma Co-orientador: Prof. Dr. Aristeu da Silveira Neto

Durante a elaboração deste trabalho, o autor recebeu apoio financeiro da Capes. 


\title{
Simulação Numérica Bidimensional da Interação Fluido-Estrutura através do Método Físico Virtual
}

\author{
Este exemplar corresponde à redação final devidamente \\ corrigida e defendida por Santos Alberto Enriquez Remigio \\ e aprovada pela comissão julgadora.
}

São Paulo, 05 de Setembro de 2005.

Banca examinadora

Prof. Dr. Alexandre Megiorin Roma

IME-USP

Prof. Dr. Aristeu da Silveira Neto

FEMEC-UFU

Profa. Dra. Ana Lúcia Fernandes de Lima e Silva

FEMEC-UFU

Prof. Dr. Antonio Castelo Filho

ICMC-USP São Carlos

Prof. Dr. Sérgio Said Mansur

DEM-UNESP de Ilha Solteira 
....A MIS PADRES. 


\section{Agradecimentos}

Agradeço a Deus por tudo o Ele que fez por mim.

Agradeço aos meus pais e irmãos pelo grande amor e à toda a minha familia pelo grande apoio.

Agradeço ao meu orientador Prof. Roma pela orientação, conselhos e pela sua preocupação e paciência.

Agradeço ao meu co-orientador Prof. Aristeu por todo o apoio e preocupação durante o desenvolvimento deste trabalho.

Agradeço ao Prof. Héctor Merklen, Profa. Helena, Profa. Joyce, Prof. Luis Carlos, Prof. Angela Weiss, Prof. Nelson Kuhl, Prof. Oscar Vilcachagua, Prof. Saulo Rabello e a todos os professores do IME por todo o apoio durante o doutorado.

Ao LCCA-Laboratório de Computação Científica Avançada do Centro de Computação Electrônica da Universidade de São Paulo, que permitiu a realização das simulações. Ao analista Francisco Ribacionka pela atenção e presteza.

Aos amigos da UFU:Ana Lúcia e Sandro, Elie e Luana, Israel, José Eduardo e seus pais, Juliano e Marla, Luis Alberto e Tatiana, Manuel, Millena, Rubens, Tumialan por todo o apoio e bons momentos compartilhados.

À Vera pela grande amizade, pelos bons momentos compartilhados e pelos ensinamentos de perseverança e coragem.

Ao meu melhor amigo Nelson pela grande amizade, apoio e paciência.

Aos meus amigos: Antonio e Josefa, Alice, Domingo, Julio e Ruth, Luis, Maité e Fernando, Mariela, Marina, Mary Luz, Mario Salles, Natalia, Nestor e Sandra, Nubia, Olga, Paula, Rocio Bendezu, Rocio Olivares, Rudimar, Sandra e Cristian, Said, Victoria e Leo.

A todos meus amigos argentinos, bolivianos, brasileiros, colombianos e peruanos que se aqui eu colocasse o nome de todos, não iria terminar de listá-los.

Aos funcionários do IME por toda a sua disposição nos momentos em que mais precisei. 


\section{Resumo}

Problemas de escoamentos turbulentos sobre geometrias móveis e complexas são muito frequentes em engenharia e também em outras áreas correlatas, por exemplo, sistemas de bombeamento, compressores, motores de combustão interna, e problemas biológicos.

As atuais metodologias para a solução desta classe de problemas apresentam grande potencial mas também grandes desafios e deficiências. A metodologia de fronteira imersa, em desenvolvimento por vários grupos de pesquisa, está se apresentando como uma ferramente alternativa com grandes potenciais. Lima e Silva et al. [25] e Arruda [1], propuseram uma nova forma de se avaliar o campo de forças que caracteriza essa metodologia. Simulações apresentadas pelos autores foram referentes à interação de um fluido com corpos móveis de velocidades prescritas.

Neste trabalho apresentam-se aplicações da metodologia da fronteira imersa com cálculo do campo de força dada por Lima e Silva et al. [25] em problemas de interação fluido e estrutura rígida de velocidade não prescrita. Foram feitas adaptações e novas implementações no código desenvolvido por estes autores. Equações do corpo rígido foram acrescentadas e a forma de resolver as equações do fluido foram modificadas. O conjunto de equaçoes do sistema: Navier-Stokes (escoamento incompressível) e Newton-Euler (corpo rígido) foram resolvidas através do Método de Runge-Kutta de segunda ordem explícito no tempo e diferenças finitas centradas de segunda ordem no espaço. Em cada passo no tempo as equações do fluido são resolvidas por intermédio do método de projeção.

O código computacional foi validado atráves da comparação com a solução analítica do problema do escoamento de Couette circular. Este código também foi aplicado na simulação de um pêndulo cilíndrico bidimensional em um meio fluido parado. Apartir destes resultados avançou-se para uma aplicação preliminar de um sistema de bombeamento composto de cavidade e válvulas.

Os resultados obtidos apontam para o importante potencial desta metodologia no que se refere à modelagem matemática e à simulação numérica de escoamentos turbulentos em sistemas de bombeamento com fronteiras rígidas móveis e escoamentos complexos.

Palavras chaves: Método da Fronteira Imersa; Modelo Físico Virtual; interação fluidoestrutura; escoamentos complexos; sistemas de bombeamento; diferenças finitas; método de projeção explícito. 


\begin{abstract}
Turbulent flows past complex, mobile obstacles occur often in engineering, physics, biological problem among other areas, e.g. internal combustion engines and flow past artificial heart valves.

The immersed boundary methodology offers a powerful tool to handle such problems and it is currently under development by several research groups. Lima e Silva et al. [25], and Arruda [1], proposed a new approach for evaluating the force field term, wich characterize this methodology. The simulations presented in their works refer to the dynamical, one-way interaction between an incompressible flow and mobile immersed strutures whose velocity is prescribed. In the present work, an extension of the immersed boundary methodology capable of handling the dynamical, two-way interaction between an incompressible flow and a rigid immersed struture (non-prescribed velocity) is described. The equations of motions, composed by the incompressible Navier-Stokes and by the Newton-Euler equations are solved numerically based on a Projection Method employing an explicit second-order Runge-Kutta Method for the time integration. The computational code was validated by comparison with several problems with known solution (either exactly or numerically, e.g. the circular Couette flow problem). This methodology is applied for performing the numerical simulation of the simple pendulum immersed in a fluid, and the flow in a complex geometry given by a channel, valves and a moving bottom.
\end{abstract}

Key words: Immersed boundary method; physical virtual model; fluid-struture interaction; finite difference method; explicit projection method; complex flows. 


\section{Índice}

Lista de Figuras

V

Lista de Tabelas viii

1 Introdução 1

1.1 Apresentação do trabalho . . . . . . . . . . . . . . . . . . 3

2 Revisão da literatura 4

2.1 Generalidades . . . . . . . . . . . . . . . . . . 5 5

2.2 Metodologias baseadas no uso de condição de contorno interna . . . . . 7

2.3 Metodologias baseadas no método da fronteira imersa . . . . . . . . . . . . 9

2.3.1 Termo forçante de Peskin [34] . . . . . . . . . . . . . . 10

2.3.2 Termo forçante de Goldstein et al. [14] . . . . . . . . . . . . . . 13

2.3.3 Termo forçante de Mohd-Yusof [30] . . . . . . . . . . . . . . . 14

2.3.4 Termo forçante de Lima e Silva et al. [25] . . . . . . . . . . 16

2.3.5 Imposição da presença da interface através de células fantasmas . . 18

2.3.6 Imposição da presença da interface através da reconstrução das células cortadas pela interface . . . . . . . . . . . . . . . 19

2.3.7 Imposição da presença da interface através de um termo do tipo multiplicador de Lagrange . . . . . . . . . . . . . . . . . 21

2.4 Resumo sobre as metodologias baseadas no MFI . . . . . . . . . . . . . 22

3 Modelagem matemática $\quad 26$ 
3.1 Modelo Físico Virtual . . . . . . . . . . . . . . . . . . . . . . . . . . 26

3.2 Função indicadora . . . . . . . . . . . . . . . . . . . . . . . . 32

3.3 Modelagem da turbulência: SGE . . . . . . . . . . . . . . . . 33

3.3.1 Modelagem sub-malha: modelo de Smagorinsky . . . . . . . . . . . 35

4 Solução numérica da modelagem matemática 36

4.1 Solução das equações de Navier-Stokes: equação de Poisson para a pressão e método de projeção . . . . . . . . . . . . . . . . . . . 37

4.2 Métodos de projeção: explícitos e semi-implícitos . . . . . . . . . . . . 38

4.2.1 Um método explícito de primeira ordem no tempo . . . . . . . . . . 39

4.2.2 Um método explícito de segunda ordem no tempo . . . . . . . . . . 40

4.2 .3 Método semi-implícito de segunda ordem no tempo . . . . . . . . 41

4.3 Discretizações adotadas . . . . . . . . . . . . . . . . . . . . . . . 42

4.3 .1 Discretização no espaço . . . . . . . . . . . . . . . . . . 42

4.3.2 Discretização temporal da densidade de força lagrangiana . . . . . 43

4.3 .3 Condição de estabilidade . . . . . . . . . . . . . . . . 46

4.4 Condições de contorno numéricas . . . . . . . . . . . . . . . . 47

4.5 Algoritmo de Runge-Kutta para a interação fluido-estrutura . . . . . . . . 48

5 Resultados numéricos $\quad 50$

5.1 Preliminares: normas e estudo de convergência . . . . . . . . . . . . . 50

5.2 Validação do método de projeção explícito . . . . . . . . . . . . . . . 52

5.2 .1 Caso 1: Condição de Dirichlet para a velocidade . . . . . . . . . 53

5.2.2 Caso 2: Condição Neumann para a velocidade . . . . . . . . . . . 53

5.3 Validação do MFI/MFV: Escoamento de Couette Circular . . . . . . . . . 54

5.4 Pêndulo imerso em um fluido . . . . . . . . . . . . . . . . . 58

5.5 Canal-cavidade com válvulas cilíndricas . . . . . . . . . . . . . . 65

5.5 .1 Descrição das condições do problema . . . . . . . . . . . . . 66

5.5 .2 Solução numérica através do MFI/MFV . . . . . . . . . . . 68

5.5.3 Experiência 1: movimento horizontal livre dos cilindros . . . . . . 68

5.5.4 Experiência 2: movimento dos cilindro limitados mecanicamente . . 71

6 Conclusão $\quad 85$ 
$\begin{array}{ll}\text { A Discretização no espaço } & 87\end{array}$

A.1 Discretização dos termos advectivos . . . . . . . . . . . . . . . . 87

A.2 Discretização do gradiente de pressão . . . . . . . . . . . . . . . . 88

A.3 Discretização dos termos difusivos . . . . . . . . . . . . . . . . . . . 88

B Condições de contornos numéricas para o passo de projeção 90

B.1 Método de projeção explícito de segunda ordem . . . . . . . . . . . . . . 90

B.1.1 Considerações adotadas na aplicação do RKE2 . . . . . . . . . . . 91

B.2 Condições de contornos numéricas para o passo de projeção . . . . . . . . . 94

B.3 Ordem de aproximação da velocidade no contorno . . . . . . . . . . . . . 94

$\begin{array}{ll}\text { Referências Bibliográficas } & 100\end{array}$ 


\section{Lista de Figuras}

1.1 Válvula artificial mecânica bola em gaiola usada no coração. . . . . . . . . 1

2.1 Domínio retangular bidimensional contendo um corpo imerso em um fluido. 5

2.2 (a) Domínio real para o sistema-fluido estrutura; (b) Malha cartesiana para o novo domínio do fluido e malha lagrangiana para o contorno do corpo. . 9

2.3 Representação da fronteira imersa devido ao uso do delta de Dirac no processo de espalhamento da força lagrangiana. . . . . . . . . . . . . . . . 12

2.4 Exemplo do ponto de aplicação do termo forçante e pontos associados no cálculo da velocidade $\overline{\mathbf{v}}$ para o termo forçante [52] . . . . . . . . . . . 15

2.5 Volume de controle ao redor do ponto lagrangiano $\mathrm{X}_{k}$ para a aplicação do balanço da quantidade de movimento. . . . . . . . . . . . . . . 17

2.6 (a) Exemplo de um domínio computacional com uma fronteira imersa, $\mathbf{x}$, ponto no domínio físico e $\Delta$, células fantasmas do domínio; (b) esquema de pontos usados para avaliar a variável localizada na células fantasma G [52]. 19

2.7 Exemplos de células trapezoidais ao redor da interface imersa [51]. . . . . . 20

2.8 Exemplo do estêncil usado para interpolar os fluxos nos lados do trapézio

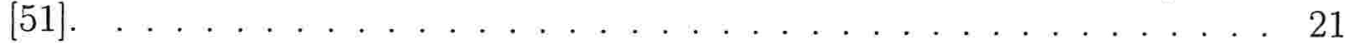

3.1 Esquema para a interpolação da pressão e da velocidade. . . . . . . . . . . . 29

3.2 Exemplo da influência da contribuição de pontos forçantes lagrangianos em pontos eulerianos. . . . . . . . . . . . . . . 30

4.1 Típica célula MAC: índice fracionário (esquerda) e adoção de índices inteiros para o processo de programação computacional (direita) . . . . . . . . . 43 
5.1 Plano transversal " $\Omega$ " ao escoamento de Couette circular (a); domínio bidimensional, $\Omega$, considerado na modelagem do MFV (b). . . . . . . . 55

5.2 Escoamento de Couette circular: campo de pressão (malha $60 \times 60$ ). . . . . 59

5.3 Escoamento de Couette circular: linhas de corrente (malha $60 \times 60)$. . . . . 59

5.4 Pêndulo imerso em uma caixa contendo um fluido. . . . . . . . . . . . . . . 60

5.5 Pêndulo imerso num fluido: história temporal do ângulo $\theta$ (rad) . . . . . . . 62

5.6 Pêndulo imerso num fluido: história temporal da velocidade angular $w$. . 63

5.7 Pêndulo imerso em um fluido incompressível: história temporal do número de Reynolds. . . . . . . . . . . . . . . . . . . . . . . 63

5.8 Módulo da força total fluidodinâmica $\mathbf{F}_{\mathrm{D}}$ atuando no corpo (dinas). . . . . 64

5.9 Módulo da diferenca entre a força total atuando na direção do movimento e as contribuições $\mathbf{F}_{\text {WE }}$ e $\mathbf{F}_{\mathrm{D}} \ldots \ldots \ldots \ldots$. . . . . . . . . . 64

5.10 Representação geométrica do conjunto canal-cavidade contendo dois cilindros imersos (dimensões em função do comprimento do pistão, L). Os pontos A e B são pontos de referência a serem usadas com o cilindro do lado esquerdo e do lado direito, respectivamente. . . . . . . . . . . . 65

5.11 Velocidade periódica para o fundo móvel. . . . . . . . . . . . . 66

5.12 História temporal do número de Reynolds para a cavidade com fundo móvel

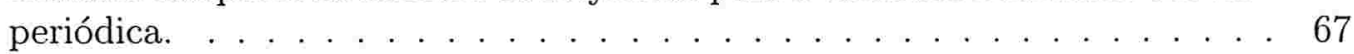

5.13 Ângulo formado pelo contato do cilindro com o vértice da contração e a linha horizontal que passa pelo centro de massa. O comprimento linf é o valor mínimo da coordenada $x$, do centro de massa do cilindro com respeito ao vértice da contração . . . . . . . . . . . . . . . . .

5.14 Movimento livre dos cilindos: abscissas relativas do centro de massa dos cilindros ( $\mathrm{xcm} 1$ e $\mathrm{xcm} 2$ ) aos pontos A e B das contrações; altura do fundo móvel da cavidade, ycav; valor mínimo para a abscissa dos cilindros, linf. $\left(\rho_{c}=0,5 \rho\right.$ e $\left.v_{0}=0,9 m / s\right) \ldots \ldots \ldots \ldots \ldots$

5.15 Movimento livre dos cilindos: abscissas relativas do centro de massa dos cilindros ( $\mathrm{xcm} 1 \mathrm{e} \mathrm{xcm} 2$ ) aos pontos $\mathrm{A}$ e $\mathrm{B}$ das contrações; altura do fundo móvel da cavidade, ycav; valor mínimo para a abscissa dos cilindros, linf. $\left(\rho_{c}=1,5 \rho\right.$ e $\left.v_{0}=0,9 \mathrm{~m} / \mathrm{s}\right)$.

5.16 Exemplo da localização de limitantes mecânicos para o movimento do centro de massa do cilindro imerso. . . . . . . . . . . . . . . . . .

5.17 Movimento dos cilindos limitados mecanicamente: abscissas relativas do centro de massa dos cilindros ( $\mathrm{x} \mathrm{cm} 1 \mathrm{e} \mathrm{xcm} 2)$ aos pontos $\mathrm{A}$ e $\mathrm{B}$ das contrações; altura do fundo móvel da cavidade, ycav; linhas horizontais inferior e superior, representam o valor de LimI e LimS, respectivamente $\left(\rho_{c}=1,5 \rho\right.$

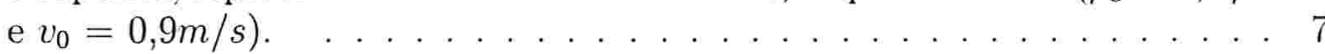


5.18 Movimento dos cilindos limitados mecanicamente: $\mathrm{X}_{c m 1}=\mathrm{X}_{c m 2}=(0,018$,

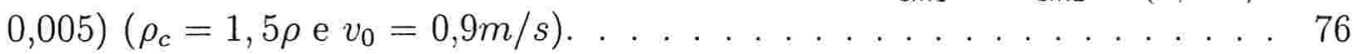

5.19 Movimento dos cilindos limitados mecanicamente: $\mathbf{X}_{c m 1}=(0,003,0,005)[\mathrm{m}]$ e $\mathbf{X}_{c m 2}=(0,009,0,005)[\mathrm{m}]\left(\rho_{c}=1.5 \rho\right.$ e $\left.V_{0}=1.45 \mathrm{~m} / \mathrm{s}\right) \ldots 77$

5.20 Movimento dos cilindos limitados mecanicamente: $\mathbf{X}_{c m 1}=(0,003,0,005)[\mathrm{m}]$ e $\mathrm{X}_{c m 2}=(0,009,0,005)[\mathrm{m}]\left(\rho_{c}=1.5 \rho\right.$ e $\left.V_{0}=1.45 \mathrm{~m} / \mathrm{s}\right)$. Espaço de separação entre a cavidade e a fronteira inferior do domínio duas vezes maior ao da experiência anterior (Figura 5.19). . . . . . . . . . . . . 77

5.21 Escoamento no interior de um conjunto canal-cavidade com fundo móvel contendo dois cilindros imersos. Campos de pressão para o intervalo de tempo $0<t \leq 0,1, \Delta t=0,01 s$.

5.22 Campos da norma da força euleriana para o intervalo de tempo $0<t \leq 0.1$, $\Delta t=0,01 s$.

5.23 Campos da força euleriana: componente vertical durante o intervalo de tempo $0<t \leq 0,1, \Delta t=0,01 s$.

5.24 Escoamento no interior de um conjunto canal-cavidade com fundo móvel contendo dois cilindros imersos. Campos de velocidade: componente horizontal para o intervalo de tempo $0<t \leq 0.1, \Delta t=0,01 \mathrm{~s} . \ldots \ldots$

5.25 Escoamento no interior de um conjunto canal-cavidade com fundo móvel contendo dois cilindros imersos. Campos da velocidade: componente vertical para o intervalo de tempo $0<t \leq 0,1, \Delta t=0,01 s . \ldots \ldots \ldots$

B.1 $u_{i}, v_{i}, p_{i}$ : variáveis internas; $u_{b}, v_{b}$ : variáveis fronteira; $u_{g}, v_{g}, p_{g}$ : variáveis

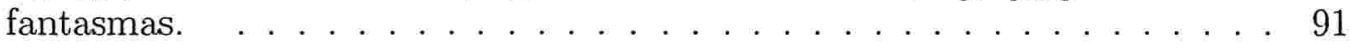

B.2 Indezação usada na discretização. . . . . . . . . . . . . . . . . . . . 92

B.3 Pontos de aplicação da condição de contorno para a velocidade em uma malha MAC. 


\section{Lista de Tabelas}

5.1 Método de projeção com condições de contorno do tipo Dirichlet para a velocidade: erros cometidos no campo de velocidade e razão entre eles, no

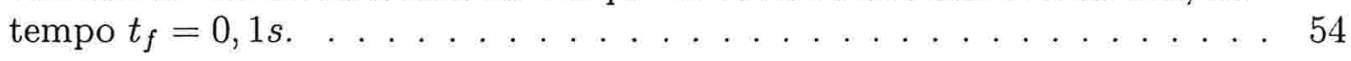

5.2 Método de projeção e condições de contorno do tipo Neumann para a velocidade: erros cometidos, no campo de velocidade, e razão entre eles no

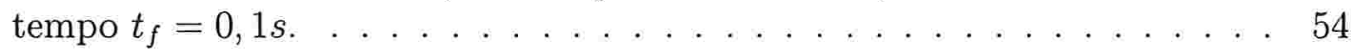

5.3 Escoamento de Couette circular: tamanho das malhas eulerianas e lagrangianas . . . . . . . . . . . . . . . . . . 57

5.4 Erros entre a solução exata e numérica para o escoamento de Couette utilizando MFI/MFV . . . . . . . . . . . . . . . . . . 57

5.5 Erro do máximo entre a velocidade lagrangiana e a velocidade do fluido na respectiva malha lagrangiana obtidos através do MFV. . . . . . . . . . . 58 


\section{CAPÍtulo 1}

\section{Introdução}

Interações fluido-estrutura são fenômenos físicos presentes na natureza.

Alguns exemplos de interação fluido-estrutura são: o sangue com as válvulas naturais ou artificiais do coração humano (Figura 1.1), a sedimentação de partículas em suspensão em um fluido, o ar e um avião em vôo, o processo de injeção de um fluido em moldes nas indústrias alimentícias e siderúrgicas, etc. O estudo da dinâmica dos fluidos desses e de outros processos físicos permite prever possíveis consequências não desejadas para adotar medidas que auxiliem a otimizar o desempenho das interações fluido-estrutura. Tal estudo pode ser feito de duas formas: experimental e teórico.

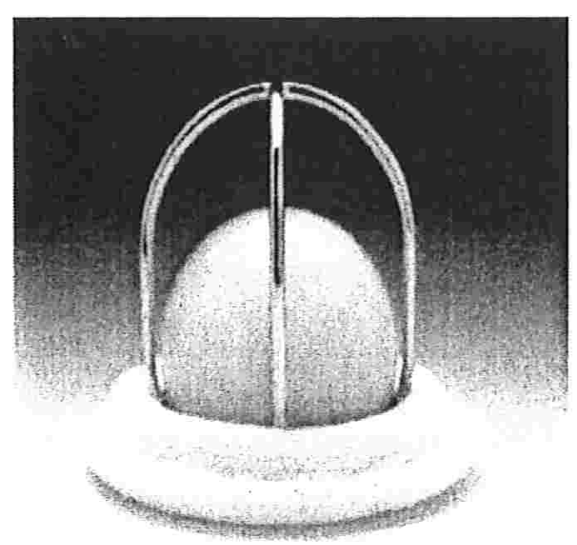

Figura 1.1: Válvula artificial mecânica bola em gaiola usada no coração.

Ensaios experimentais em laboratório, como testes em túneis de vento e tanques de água representam uma abordagem para o estudo da dinâmica dos fluidos. Estes oferecem 
resultados mais reais, mas nem toda experiência pode ser reproduzida em laboratório com baixo custo e com tempo suficientemente curto.

O estudo teórico está baseado na análise das soluções das equações de um modelo matemático associado ao fenômeno físico de estudo. Estas soluções podem ser encontradas analiticamente e/ou numéricamente. Para alguns modelos matemáticos simples (por exemplo, escoamento de Poiseuille) soluções analíticas são conhecidas, porém, os modelos matemáticos para problemas de interação fluido-sólido são em geral complexos e com solução fechada desconhecida, devido à natureza não linear das equações que compõem o modelo matemático. Neste caso, métodos numéricos é o caminho a ser seguido na procura da solução para estes problemas.

O estudo teórico através da solução numérica das equações que modelam o fenômeno físico oferece um ambiente de testes mais flexível já que muitas experiências podem ser feitas para um mesmo problema variando-se os valores dos parâmetros, das condições inicial e de contorno. As informações são calculadas no domínio total de estudo (diferentemente do caso experimental), o que permite uma melhor análise do escoamento em diferentes regiões do domínio e a possibilidade de simular problemas que não são possíveis de reproduzir em laboratório. Este tipo de estudo computacional da dinâmica dos fluidos é conhecido como Dinâmica de Fluidos Computacional (DFC). Estas vantagens da DFC não implicam que a análise experimental seja deixada de lado: é muito comum o uso das duas técnicas simultaneamente [31].

A DFC é uma alternativa que permite estudos mais abrangentes desde que o modelo matemático seja adequado e as técnicas computacionais possam resolvê-lo com precisão, eficiência e robustes. A vantagem do uso da DFC está na possibilidade de se desenvolver muitas experiências para um mesmo fenômeno, com baixo custo, assim como de se obter informações em regiões de difícil aceso, como é o caso dos experimentos em laboratório.

A procura por metodologias numéricas eficientes e robustas para o estudo da interação fluido-estrutura propulsionaram o surgimento de uma grande variedade de metodologias. Cada uma delas apresentam algumas vantagens e desvantagens e, existe um constante esforço para aperfeiçoá-las.

No Laboratório de Transferência de Calor e Massa e Dinâmica dos Fluidos (LTCM) da Faculdade de Engenharia Mecânica da Universidade Federal de Uberlândia foi desenvolvida uma metodologia para estudar problemas de interação fluido-estrutura rígida. Esta metodologia está baseada no Método da Fronteira Imersa (MFI) (Seção 2.3) que impõe a presença da estrutura através de um campo de forças. O LTCM-UFU apresentou 
uma nova forma de calcular o campo de forças denominado Modelo Físico Virtual (MFV). No presente trabalho, esta metodologia é estendida para aplicação em outras classes de problemas.

\subsection{Apresentação do trabalho}

A aplicabilidade do MFI/MFV foi mostrada em alguns trabalhos para problemas bidimensionais com estruturas rígidas em repouso ou com movimento prescrito. O objetivo deste trabalho é estudar o comportamento do MFI/MFV para problemas de interações fluido-estrutura rígida gerais, isto é, a velocidade da estrutura rígida não é conhecida a priori, mas determinada durante o processo de integração das equações do sistema fluidoestrutura. Para isto, foi utilizado como código base o código desenvolvido por Lima e Silva [25], ao qual foram acrescentadas algumas subrotinas, a fim de:

- aproximar a condição de contorno por diferenças finitas de segunda ordem;

- validar o método de solução das equações do fluido para problemas com solução analítica conhecida;

- modelar novas geometrias;

- resolver as equações da dinâmica das estruturas rígidas.

Este trabalho está organizado da seguinte forma: uma revisão da literatura específica é apresentada no Capítulo 2. No Capítulo 3, é apresentado o modelo matemático usado para o sistema fluido-estrutura rígida. No Capítulo 4 é apresentada a metodologia numérica para resolver as equações do sistema fluido-estrutura rígida. Resultados dos testes de validação do código sem fronteira imersa e de problemas de aplicação do MFV são o conteudo no Capítulo 5. A conclusão e planos futuros são apresentados no Capítulo 6 . Alguns apêndices foram incluídos no final deste trabalho. O Apêndice A descreve as aproximações no espaço para a condição de contorno e para os operadores das equações de Navier-Stokes adotados. No Apêndice B é apresentada uma demonstração do Lema 4, usado para aproximar a condição de contorno numérica do passo de projeção da solução das equações de Navier-Stokes. 


\section{Revisão da literatura}

Um modelo matemático para a interação fluido-estrutura deve considerar as equações para o fluido, as equações para a estrutura e as relações de acoplamento entre elas, que determinam a interação total. Essas relações de acoplamento são dadas pelas forças de ação e reação existentes entre o fluido e a estrutura. No caso da ação do corpo sobre o fluido, estas relações de acoplamento podem ser vistas como um tipo de condição de contorno interna para o fluido. A ação do fluido sobre o corpo é dada pelas forças fluidodinâmicas que este exerce na estrutura e as quais são responsáveis pelo movimento de translação e rotação da estrutura. Na Seção 2.1, são apresentadas as equações para o sistema fluidoestrutura. Soluções analíticas não são possíveis e, portanto, técnicas computacionais são empregadas para resolvê-las.

A procura por técnicas computacionais eficientes e robustas para estudar a interação fluido-estrutura motivou o desenvolvimento de diversas metodologias. Estas metodologias apresentam vantagens e desvantagens e existe um constante esforço para aperfeiçoá-las. Na Seção 2.2 são apresentadas algumas metodologias que imponhem a presença da estrutura no fluido por intermédio de condição de contorno nas equações do fluido. Na Seção 2.3 são apresentadas algumas metodologias que usam o Método da Fronteira Imersa e imponhem a presença atráves de um termo forçante ou modificação da solução ao redor da fronteira da estrutura.

Na Seção 2.4 apresentam-se algumas observações referentes aos métodos baseados no Método da Fronteira Imersa. 


\subsection{Generalidades}

As equações para o modelo matemático da interação fluido-corpo rígido são deduzidas a partir dos princípios físicos que governam o fluido e o corpo rígido. Na Figura 2.1, apresenta-se uma região retangular, $\Omega \subset \mathrm{R}^{2}$, ocupada por um fluido e um corpo rígido. A região ocupada pelo corpo é denotada por $B(t)$ e aquela ocupada pelo fluido é $\Omega \backslash B(t)$. O contorno do corpo (ou interface fluido-estrutura) é denotado por $\gamma$ e o contorno do domínio do sistema $(\Omega)$ é denotado por $\Gamma$.

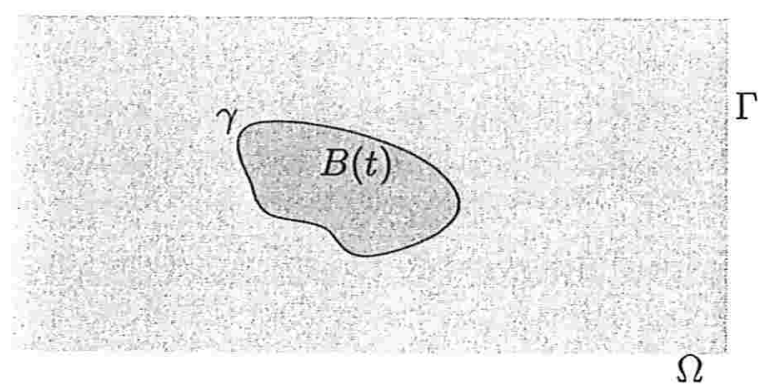

Figura 2.1: Domínio retangular bidimensional contendo um corpo imerso em um fluido.

Principíos físicos que governam o fluido são válidos nas regiões que o mesmo está ocupando. Estes princípios físicos são: balanço da massa, balanço da quantidade de movimento (Segunda Lei de Newton), e balanço da energia (Primeira Lei da Termodinâmica).

Para o caso de escoamentos incompressíveis e isotérmicos, as equações baseadas nos princípios físicos (balanço da massa e balanço de quantidade de movimento) são:

$$
\begin{aligned}
\rho\left(\frac{\partial \mathbf{u}}{\partial t}+\mathbf{u} \cdot \nabla \mathbf{u}\right) & =\nabla \cdot \sigma \\
\nabla \cdot \mathbf{u} & =0
\end{aligned}
$$

onde $\sigma=-p \mathbf{I}+2 \mu \mathbf{d}$ é o tensor das tensões e $\mathbf{d}=\frac{\left(\nabla \mathbf{u}+\nabla^{t} \mathbf{u}\right)}{2}$ é o tensor das taxas de deformação; $\mathbf{u}=\mathbf{u}(\mathbf{x}, t)$ ( $\mathrm{x}$ é a posição no espaço e $t$ é o tempo), representa a velocidade do fluido, e a função escalar $p=p(\mathbf{x}, t)$, representa o campo de pressão; os parâmetros físicos $\rho$ e $\mu$, constantes no espaço e no tempo, são respectivamente a massa especifica e a viscosidade dinâmica.

O sistema de equações (2.1-2.2) é um problema de valor inicial e de contorno. Para resolvê-lo, é preciso uma condição inicial e condições de contorno adequadas. 
Para o exemplo de um corpo imerso em um fluido (Figura 2.1) a condição inicial é dada por

$$
\mathrm{u}(\mathrm{x}, 0)=\mathrm{u}_{0} \quad \text { em } \quad \Omega \backslash B(0),
$$

e as condições de contorno são dadas por: (1) na fronteira do corpo, $\gamma$; e (2) no contorno de $\Omega, \Gamma$.

A condição de contorno em $\Gamma$ depende do problema em análise e será denotado por:

$$
\mathrm{CC}(\mathrm{u}(\mathrm{x}, t))=\mathbf{h}(\mathrm{x}, t) .
$$

A expressão $\mathrm{CC}(\mathrm{u})$ pode representar, entre outras, uma condição de contorno do tipo Dirichlet e/ou Neumann e, h é o respectivo valor associado à condição de contorno para u.

A condição de contorno na interface, $\gamma$, é a condição de contorno do tipo Dirichlet para a velocidade, isto é,

$$
\left.\mathrm{u}\right|_{\gamma}=\mathrm{V},
$$

onde $\mathrm{V}$ é a velocidade que o corpo tem no contorno.

O modelo matemático para o corpo rígido também é dado pelos princípios físicos de balanço da quantidade de momento linear e da quantidade de momento angular para um corpo rígido. Estes princípios determinam a dinâmica do corpo rígido: translação e rotação.

Devido ao fato que corpo é rígido, todos os pontos transladam-se por igual. Portanto, para referir-se ao movimento de translação é suficiente fixar um ponto no corpo e definir o movimento de translação com respeito a tal ponto. O movimento de rotação é devido ao torque resultante que as forças externas exercem com respeito a um ponto de aplicação no corpo.

Neste trabalho, assume-se que o centro de massa do corpo é o ponto usado para definir tanto o movimento de translação quanto o ponto de aplicação do torque no corpo. As equações do modelo matemático para a dinâmica de um corpo rígido com massa $M_{c} \mathrm{e}$ momento de inércia $I_{c}$ são dadas por [13]:

$$
\begin{aligned}
M_{c} \frac{d \mathbf{V}_{c}}{d t} & =\mathbf{G}_{c}+\mathbf{F}_{f c}, \\
I_{c} \frac{d}{d t}(\mathbf{w}) & =\mathbf{T}_{f c},
\end{aligned}
$$

onde: $\mathrm{V}_{c}$ e w são a velocidade de translação do centro de massa e a velocidade angular do corpo com respeito ao centro de massa, respetivamente. $\mathbf{G}_{c}$ é o peso do corpo, $\mathbf{F}_{f c}$ 
é a força total externa atuando no corpo e $\mathbf{T}_{f c}$ é o torque total produzido pelas forças externas no corpo.

A posição do centro de massa, $\mathbf{X}_{c}$, e do ângulo de rotação, $\theta_{\boldsymbol{c}}$, podem ser determinadas usando-se as equaçōes cinemáticas:

$$
\begin{aligned}
& \frac{d \mathbf{X}_{c}}{d t}=\mathbf{V}_{c} \\
& \frac{d \theta_{c}}{d t}=w
\end{aligned}
$$

Quando um corpo encontra-se imerso em um fluido, as forças que o fluido exerce no corpo rígido podem ser determinadas com base em um tensor de tensões. Tais forças de ação do fluido no corpo são denominadas forças fluidodinâmicas e, em conjunto com o peso do corpo, determinam a dinâmica do corpo rígido. A expressão para a força resultante e o torque resultante devido à ação do fluido no corpo estão dados por:

$$
\begin{aligned}
\mathbf{F}_{f c} & =\int_{\partial B(t)} \sigma \cdot \mathbf{n} d s \\
\mathbf{T}_{f c} & =\int_{\partial B(t)}\left(\mathbf{x}-\mathbf{X}_{c}\right) \times \sigma . \mathbf{n} d s .
\end{aligned}
$$

Equações (2.1-2.2) são conhecidas como as equações de Navier-Stokes e as equações (2.6-2.9) são conhecidas como as equações de Newton-Euler. Estas equações, considerandose as condições de contorno (interna e externa) similares as dadas por (2.4-2.5), são as equações do modelo matemático usado na metodologia apresentada a seguir.

\subsection{Metodologias baseadas no uso de condição de contorno interna}

Estes métodos caracterizam-se por impor a presença do corpo nas equações do fluido usando condições de contorno internas para o domínio do fluido. As equações usadas nestes métodos são (2.1)-(2.9).

Nesta metodologia usam-se malhas estruturadas ou não estruturadas para discretizar o domínio do fluido, que permitem se adaptarem ao contorno dado pela interface fluidoestrutura. O domínio do fluido pode apresentar regiões complexas devido à geometria 
que a estrutura possui e pode estar mudando de topologia conforme a dinâmica desenvolvida pelo corpo devido à interação com o fluido. Isto implica na reconstrução da malha associada ao domínio do fluido.

$\mathrm{O}$ uso de malhas estruturadas nesta metodologia pode requer o uso de sistemas de coordenadas generalizadas. As equações devem ser transformadas para este sistema e as equações discretas podem apresentar caracteríticas especiais que precisam de métodos robustos para resolvê-las. Geralmente são usadas discretizações no espaço baseadas em diferenças finitas ou volumes finitos.

Por outro lado, o uso de malhas não estruturadas permitem uma discretização mais precisa, porém, o custo de gerar uma malha não estruturada pode ser muito alto ou inviável dependendo da complexidade geométrica do domínio ocupado pelo fluido. Neste caso, as equações não precisam ser transformadas para outros sistemas. Este tipo de malha é usada em conjunção aos métodos dos elementos finitos e volume finitos para discretizar as equações do fluido.

A principal desvantagem da metodologia baseada no uso de condição de contorno interna é o custo da geração e alteração da malha para o fluido (quando possível), assim como a necessidade de métodos eficientes para resolver os sistemas lineares associados ao problema discreto resultante.

Um dos primeiros trabalhos de simulação numérica direta usando a metodologia de condição de contorno foi apresentada por Hu et al. [17]. Neste trabalho foi usado o método de elementos finitos para resolver as equações do modelo matemático em duas dimensões onde simularam a sedimentação de algumas partículas (circulares e elípticas) num canal.

Em um outro trabalho, Hu [16] usou uma generalização de um método padrão de elementos finitos de Galerkin. Esta generalização permite incorporar as equações do fluido e da partícula em uma única equação variacional para o sistema fluido-sólido, contendo ambas, velocidades das partículas e do fluido, como incógnitas primitivas. Esta formulação evita cálculo das forças fluidodinâmicas e torques pois estas passam a ser forças internas para o sistema fluido-sólido. Neste segundo trabalho foi apresentada uma simulação do escoamento de Poiseuille da mistura líquido-sólido ao longo de um canal vertical. 


\subsection{Metodologias baseadas no método da fronteira imersa}

Nesta metodologia considera-se um novo domínio para o fluido formado pela união da região ocupada pelo fluido e a região ocupada pelo corpo rígido. A imposição da presença do corpo no fluido é feita mediante a presença de um termo forçante e/ou modificação da solução das equações do fluido ao redor da interface fluido-estrutura.

Uma malha cartesiana é usada para discretizar o novo domínio do fluido e uma malha lagrangiana é usada para definir o contorno da interface. Ambas as malhas são independentes. A comunicação entre elas é feita através de regras ou fórmulas dadas pela metodologia. Figura 2.2-(a) apresenta um exemplo de um corpo imerso em um fluido. Na Figura 2.2-(b) a representação da versão discreta usada nos métodos da fronteira imersa: malha cartesiana fixa para o novo domínio do fluido e malha lagrangiana para representar o contorno da estrutura imersa.

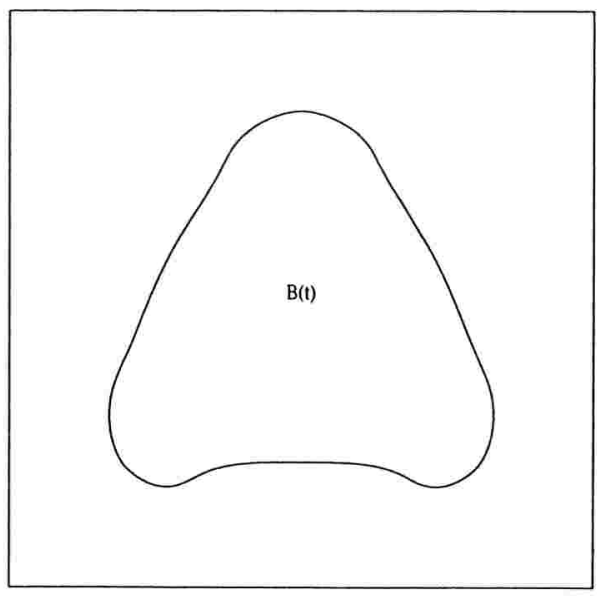

$\Omega$

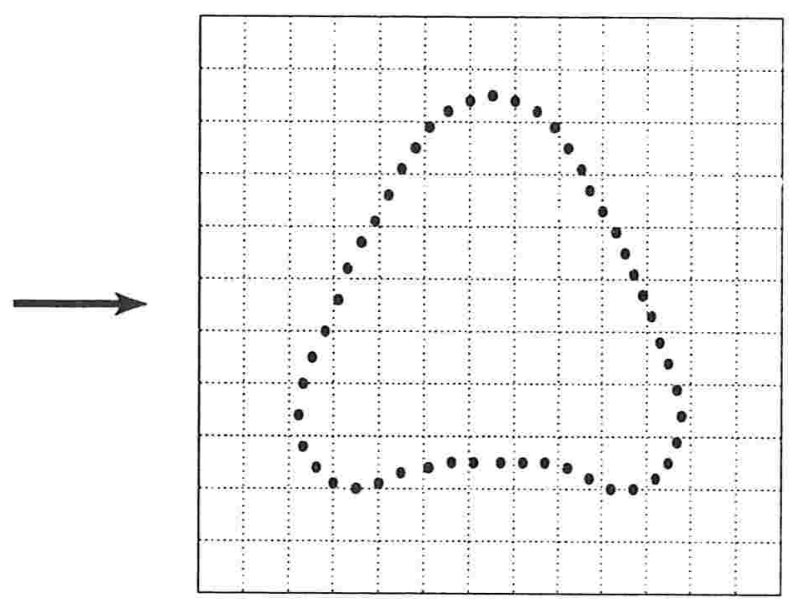

$\Omega$

Figura 2.2: (a) Domínio real para o sistema-fluido estrutura; (b) Malha cartesiana para o novo domínio do fluido e malha lagrangiana para o contorno do corpo.

Peskin [34] apresentou um modelo matemático e um método numérico, conhecido como o Método da Fronteira Imersa, para estudar o escoamento de sangue ao redor da válvula mitral. Embora a base da dedução do modelo matemático dado por Peskin 
esteja fundamentada para fronteiras com propriedades elásticas, esta é usada para simular interação fluido-estrutura rígida.

Neste trabalho, denomina-se genericamente de Método da Fronteira Imersa uma variedade de metodologias baseados na imposição da presença do corpo por intermedio de um termo forçante ou pela modificação das equações do fluido ao redor da fronteira imersa utilizando uma malha cartesiana.

Método da fronteira imersa apresentam a vantagem de serem de baixo custo computacional relativamente às metodologias que usam condições de contorno ([10]), pois o uso de uma malha cartesiana permite o uso de métodos eficientes existentes para resolver as equações discretas associadas ao fluido. Além disso, em problemas com estruturas em movimento, não é necessário uma reconstrução da malha euleriana do fluido. A desvantagem é que não há expressão exata para o termo forçante a ser usado nas equação do fluido (ou a forma correta de se modificar as equações do fluido em torno da interface) para impor corretamente a presença e ação da estrutura nas equações do fluido. Esta característica motivou o aparecemento de várias metodologias, cada uma delas mostrou bom comportamento nas experiências apresentadas pelos autores.

\subsubsection{Termo forçante de Peskin [34]}

O Método da Fronteira Imersa de Peskin [34] foi introduzido para estudar interação fluidoestrutura elástica. Nesta metodologia, a estrutura considerada é formada por fibras, cada uma contendo pontos sem massa que movimentam-se com a velocidade local do fluido, isto é

$$
\frac{\partial \mathbf{X}_{k}}{\partial t}=\mathbf{u}\left(\mathbf{X}_{k}, t\right)
$$

onde $\mathrm{X}$ é uma parâmetrização da malha lagrangiana e $\mathbf{u}\left(\mathrm{X}_{k}, t\right)$ é a velocidade do fluido no ponto lagrangiano $\mathrm{X}_{k}$.

A densidade de força euleriana é dada por

$$
\mathbf{f}(\mathbf{x}, t)=\int_{S} \mathbf{F}(\mathbf{X}(s, t), t) \delta(\mathbf{x}-\mathbf{X}(s, t)) d s
$$

onde: $\mathbf{F}(s, t)$ é a densidade de força lagrangiana; $\delta$ é a "função" delta de Dirac e $S$ é a superfície (ou contorno) da estrutura imersa.

Devido às propriedades elásticas da estrutura, a densidade de força lagrangiana tem a seguinte expressão: 


$$
\mathbf{F}(s, t)=\frac{\partial T \tau}{\partial s}(s, t) .
$$

onde $\tau$ é o vetor tangente unitário à curva e a variável $T$ é a tensão na fibra cujo valor é dado pela lei generalizada de Hooke

$$
T=T\left(\left\|\frac{\partial \mathbf{X}}{\partial s}\right\|, s, t\right)
$$

A função delta de Dirac em uma dimensão é definida como

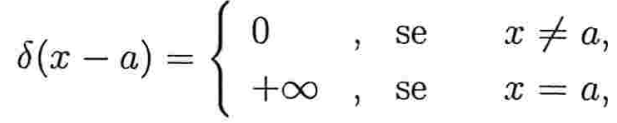

$$
\begin{aligned}
& \int_{-\infty}^{\infty} \delta(x-a) d x=1 \\
& \int_{-\infty}^{\infty} \delta(x-a) \phi(x) d x=\phi(a) \quad \phi \text { continua com suporte compacto. }
\end{aligned}
$$

No método computacional, uma aproximação suave para a função delta de Dirac é usada. No presente trabalho adotou-se a seguinte expressão para o delta de Dirac bidimensional: $\delta_{h}(x, y)=\frac{1}{h^{2}} D\left(\frac{x}{h}\right) D\left(\frac{y}{h}\right)$, onde $h=h_{x}=h_{y}$ é o espaçamento da malha $\mathrm{e}$

$$
D(r)=\left\{\begin{array}{lll}
\frac{1}{8}\left(3-2|r|+\sqrt{1+4|r|-4 r^{2}}\right) & , \text { se } & |r| \leq 1 \\
\frac{1}{8}\left(5-2|r|-\sqrt{-7+12|r|-4 r^{2}}\right) & , \text { se } 1 \leq|r| \leq 2 \\
0 & , \text { se } & |r|>2
\end{array}\right.
$$

Esta função é positiva, contínua e com suporte compacto $[-2,0,2,0]$. Outras expressões para a função $D$ (assim como as propriedades que ela deve cumprir) podem ser encontradas em [8], [35].

As equações (2.12) e (2.13) são as equações de interação entre as duas formulações: euleriana e lagrangiana. A primeira equação permite conhecer a velocidade da interface mediante um processo de interpolação. A segunda, dá a força que age no fluido devido à presença da estrutura. Neste caso um processo de espalhamento da força lagrangiana é feito. Devido ao uso de uma versão suave para a função delta de Dirac, a força lagrangiana é distribuída ao redor da interface como mostrado na Figura 2.3. Dependendo do suporte adotado para a aproximação do delta de Dirac as forças são espalhadas a duas ou mais células computacionais ao redor da interface.

O método de Peskin tem sido aplicado a uma grande variedade de problemas biológicos [35]. Sua aplicação para modelar interação fluido-sólido, embora o modelo matemático represente a interação fluido-estrutura elástica, é feita seguindo duas estrátegias. A primeira 


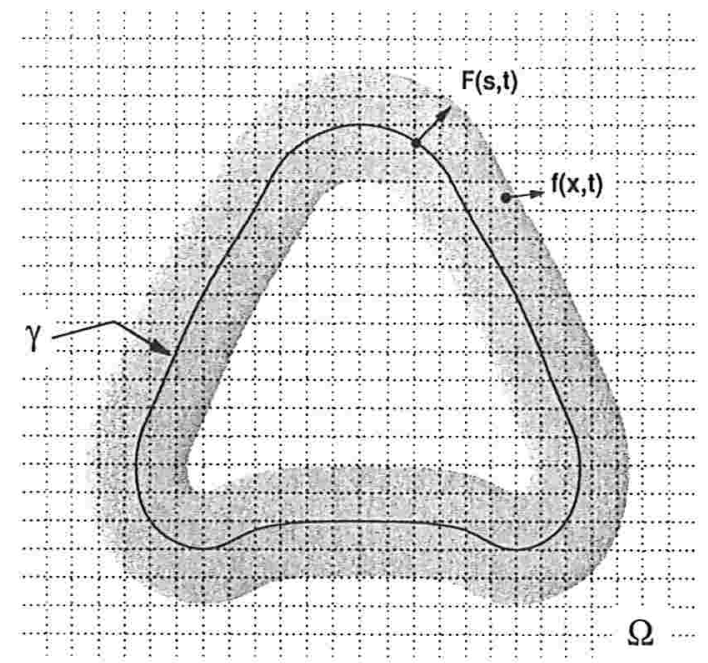

Figura 2.3: Representação da fronteira imersa devido ao uso do delta de Dirac no processo de espalhamento da força lagrangiana.

considera constantes de elasticidade altas entre os pontos lagrangianos da interface. A segunda consiste em amarrar os pontos lagrangianos da estrutura a uma posição desejada por intermédio de molas e aplicar uma força restauradora nos pontos lagrangianos dada pela lei de Hooke,

$$
\mathbf{F}\left(\mathrm{X}_{k}, t\right)=-l\left(\mathrm{X}_{k}-\mathrm{X}_{k}^{e}\right)
$$

onde $l$ representa a constante de Hooke e $\mathrm{X}_{k}^{e}$ é a posição desejada para a estrutura. O problema associado com estas estrátegias é que o sistema de equações fica mal condicionado e, para mantêr-lo estável, pequenos passos no tempo são necessários.

Lai [28] simulou o escoamento de um fluido ao redor de um cilindro estacionário utilizando a metodologia de Peskin e usou a segunda estrátegia para manter os pontos lagrangianos estacionários. Coeficientes de arrasto e de sustentação e o número de Strouhal foram calculados. Uma boa concordância com resultados de outros autores foi obtida. Lai deduziu uma expressão para o tamanho do passo no tempo,

$$
\Delta t \approx C \sqrt{\frac{h}{l}} .
$$

Enriquez-Remigio e Roma [9] apresentaram a simulação do escoamento de Poisseuille 
mediante o uso da metodologia de Peskin. Para simular a entrada e a saída de fluido no canal foram usados elementos de modelagem matemática denominados fontes e sumidouros. O perfil parabólico da velocidade foi obtido e um estudo de convergência mostraou a convergência de primeira ordem do Método da Fronteira Imersa. Valores grandes para as constantes de rígidez foram necessários para manter o corpo na posição de equilibrio desejada. Isto implicou na imposição de uma forte restrição para o passo no tempo dado por (2.20).

Uhlmann [44] usou a metodologia de Peskin e as equações de Newton-Euler para simular a queda livre de uma partícula circular em um fluido. Uma expressão para o cálculo das forças hidrodinâmicas e torque na partícula foi determinada por ele. Os resultados mostraram boa concordância com as de outros autores.

\subsubsection{Termo forçante de Goldstein et al. [14]}

Goldstein et al. [14] propuseram o Método da Fronteira Virtual para simular o escoamento de um fluido sobre corpos submersos com velocidade prescrita. Similarmente ao Método da Fronteira Imersa de Peskin, a fronteira sólida é considerada como gerador de força. Tal campo de força é modelado por uma função feedback, que relaciona a velocidade do fluido com a velocidade prescrita.

$$
\mathbf{F}\left(\mathbf{X}_{k}, t\right)=\alpha \int_{0}^{t}\left(\mathbf{u}\left(\mathbf{X}_{k}, t\right)-\mathbf{V}\left(\mathbf{X}_{k}, t\right)\right) d t+\beta\left(\mathbf{u}\left(\mathbf{X}_{k}, t\right)-\mathbf{V}\left(\mathbf{X}_{k}, t\right)\right),
$$

onde $\mathbf{u}\left(\mathrm{X}_{k}, t\right)$ é a velocidade do fluido em $\mathrm{X}_{k}$ e $\mathrm{V}$ é a velocidade prescrita do corpo. As constantes $\alpha$ e $\beta$ são negativas e devem ser ajustadas de acordo com o problema a ser simulado.

As simulações apresentadas por Goldstein et al. [14] estão no contexto dos métodos espectrais. Para evitar o processo de interpolação, as forças foram aplicadas diretamente na malha euleriana. Assim, uma fronteira do tipo escalonada é considerada, em vez de uma suave. Foram realizadas simulações de escoamentos turbulentos sobre um modelo de superfície coberta, entre outros problemas. Eles executaram uma análise de estabilidade e determinaram que quando a forçante é computada explicitamente por um esquema Adams-Bashforth, a restrição do passo no tempo é dada por

$$
\Delta t<\frac{\left(-\beta-\sqrt{-\beta^{2}-2 \alpha k}\right)}{\alpha}
$$


onde $k$ é uma constante de ordem 1 dependendo do escoamento.

Saiki et al. [39] aplicaram o método de Goldstein et al. [14] no contexto do método de diferenças finitas, com aproximação no espaço de quarta ordem. Um processo de interpolação para calcular a velocidade do fluido na fronteira e para distribuir a força da fronteira na malha euleriana foi usado. Eles simularam o escoamento de um fluido sobre um cilindro estacionário ou em movimento de rotação. Uma restrição do passo no tempo similar à equação (2.22) para o esquema de Runge-Kutta usado foi encontrado.

\subsubsection{Termo forçante de Mohd-Yusof [30]}

Mohd-Yusof [30] apresentou uma proposta para o termo forçante que deve ser acrescentado nas equações de Navier-Stokes. Este termo é baseado na versão discreta da metodologia empregada para resolver as equações de Navier-Stokes. Por exemplo, para uma aproximação de Euler progressiva da equação da quantidade de movimento

$$
\rho\left(\frac{\mathbf{u}^{\mathrm{n}+1}-\mathbf{u}^{\mathrm{n}}}{\Delta t}+(\mathbf{u} \cdot \nabla \mathbf{u})^{n}\right)=-\nabla p^{n}+\nabla \cdot[2 \mu \mathbf{d}]^{n}+\mathbf{f}^{n},
$$

o termo forçante que impõe a condição de contorno na interface $\gamma$ é dado por

$$
\mathbf{f}^{n}= \begin{cases}\rho\left(\frac{\mathbf{V}-\mathbf{u}^{n}}{\Delta t}+(\mathbf{u} \cdot \nabla \mathbf{u})^{n}\right)+\nabla p^{n}-\nabla \cdot[2 \mu \mathrm{d}]^{n}, & \text { em } \gamma \\ 0, & \text { outro caso. }\end{cases}
$$

Se os pontos lagrangianos da interface $\gamma$ coincidem com pontos eulerianos, então substituindo a equação (2.24) na equação (2.23), tem-se que: $\mathbf{u}^{n+1}=\mathrm{V}$ nos pontos requeridos. $\mathrm{Na}$ prática, os pontos da malha lagrangiana não coincidem com pontos da malha euleriana. Isto implica na necessidade de definir os pontos onde é aplicado o termo forçante e qual é o valor para a velocidade $\mathrm{V}$ a ser usada nesses pontos.

Mohd-Yusof [30] aplicou esta metodologia no contexto dos métodos espectrais. O termo forçante foi aplicado em pontos adjacentes à fronteira imersa e no interior dela. A simulação do escoamento laminar em um canal tridimensional com chicanas foi apresentada. Nesta metodologia, o termo forçante não depende de parâmetros que devam ser ajustados para impor a condição de contorno, diferentemente da metodologia de Peskin que é aplicada para corpos rígidos [28] e Goldstein et al.[14]. Mas depende da escolha dos pontos onde será aplicado o termo forçante e a velocidade adotada para esses pontos.

Fadlun et al. [10] compararam a metodologia de Goldstein et al. [14] e a de Mohd- 
Yusof [30] no contexto do método de diferenças finitas em uma malha deslocada. Experiências computacionais mostraram que, embora ambas metodologias proporcionem essencialmente os mesmos resultados, a última é subtancialmente mais eficiente, fazendo com que esta seja mais apropiada para simulações de escoamentos complexos em três dimensões. Eles escolheram o ponto de aplicação do termo forçante na primeira célula externa à interface na direção $x$ e $y$. A velocidade na primeira célula externa escolhida foi interpolada usando-se a velocidade da interface e a velocidade da segunda célula na respectiva direção. Os outros termos do termo forçante são calculados mediante valores das variáveis provenientes de um passo preditor.

Na Figura 2.4 mostra-se o ponto de aplicação do termo forçante e as velocidades usadas para interpolar a velocidade, requerida pelo termo forçante, empregada por Fadlun et al [10]. Eles apresentaram simulações, entre outras, do escoamento no interior de um sistema pistão/cilindro em altos números de Reynolds.

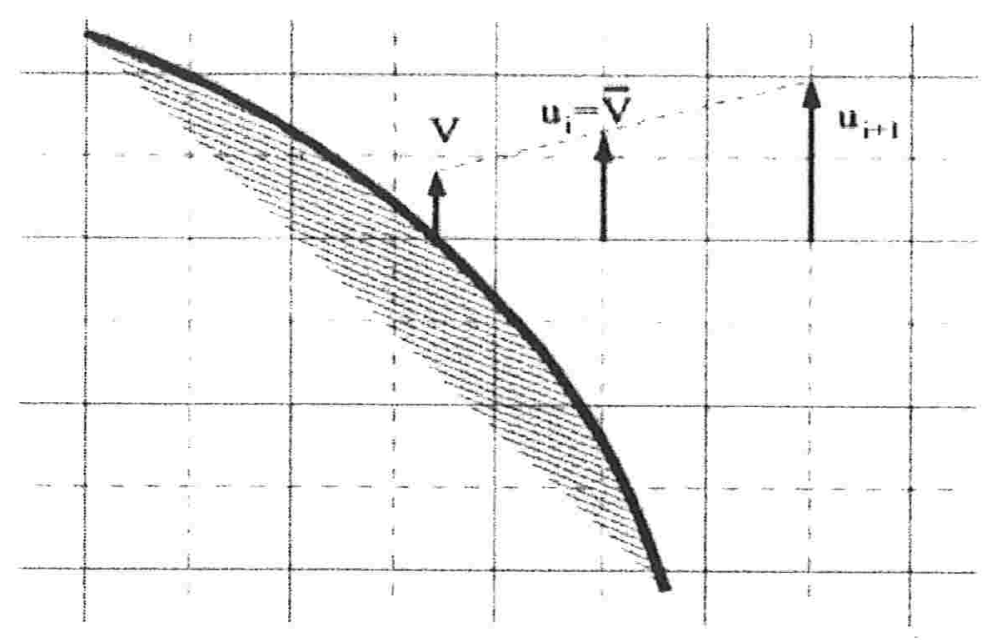

Figura 2.4: Exemplo do ponto de aplicação do termo forçante e pontos associados no cálculo da velocidade $\overline{\mathrm{v}}$ para o termo forçante [52].

Kim et al. [22] apresentaram um método baseado no termo forçante direto dado por Mohd-Yusof e em um termo fonte/sumidouro de massa. O termo forçante discreto foi usado para impor a condição de contorno e o termo fonte/sumidouro para conservação da massa em células que contêm pontos lagrangianos. Um processo de interpolação para avaliar a velocidade a ser usada no termo forçante foi apresentada. Eles apresentaram, entre outras simulações, o escoamento ao redor de um cilindro e de uma esfera. 
Balaras [2] apresentou uma metodologia para desenvolver Simulação de Grandes Escalas (SGE) com termo forçante dado por Mohd-Yusof. A diferença de Fadlun et al. [10], é a direção de aplicação dos pontos forçantes, baseada na direção do vetor normal ao contorno do corpo. Estrátegias para interpolar a velocidade são dadas. Simulação do escoamento 3D ao redor de um cilindro circular foi apresentado, entre outros.

Outras aplicações do método da forçante de Mohd-Yusof onde a estrutura tem uma velocidade prescrita, foi apresentada por Verzicco et al. [47]: para estudar o escoamento no interior de um tanque de mistura usando técnicas de fronteira imersa.

\subsubsection{Termo forçante de Lima e Silva et al. [25]}

Lima e Silva et al. [25] propuseram o Modelo Físico Virtual para simular a interação fluido-estrutura rígida. Este pertence à classe de métodos da fronteira imersa que impõe a presença da estrutura através de um termo forçante. Algumas diferenças do MFV com relação a métodos que também se baseiam em termos forçantes são:

- forma de se obter o termo forçante f: através da distribuição da força calculada na interface;

- princípio físico para se obter a densidade de força lagrangiana $\mathbf{F}$ : balanço da quantidade de movimento nos pontos lagrangianos;

- não requer esquemas especiais para localizar as malhas nas quais se calculam as velocidades e se atribuiem as forças.

Na Figura 2.5 apresenta-se um volume de fluido ao redor do ponto lagrangiano $\mathrm{X}_{k}$. Os autores consideram que nesse volume, além do fluido que rodeia ao ponto lagrangiano se tem um campo de forças devido à ação da estrutura no fluido, $\mathbf{F}\left(\mathrm{X}_{k}, t\right)$. Aplicando o balanço da quantidade de movimento nesse volume, obtiveram a seguinte expressão para a densidade de força lagrangiana:

$$
\mathbf{F}\left(\mathbf{X}_{k}, t\right)=\left(\rho\left(\frac{\partial \mathbf{u}}{\partial t}+(\mathbf{u} \cdot \nabla \mathbf{u})\right)+\nabla p-\nabla \cdot(2 \mu \mathrm{d})\right)\left(\mathbf{X}_{k}, t\right)
$$

Tal força pode ser expressa da seguinte forma

$$
\mathbf{F}\left(\mathrm{X}_{k}, t\right)=\mathbf{F}_{\mathbf{a}}\left(\mathrm{X}_{k}, t\right)+\mathbf{G}\left(\mathrm{X}_{k}, t\right)
$$




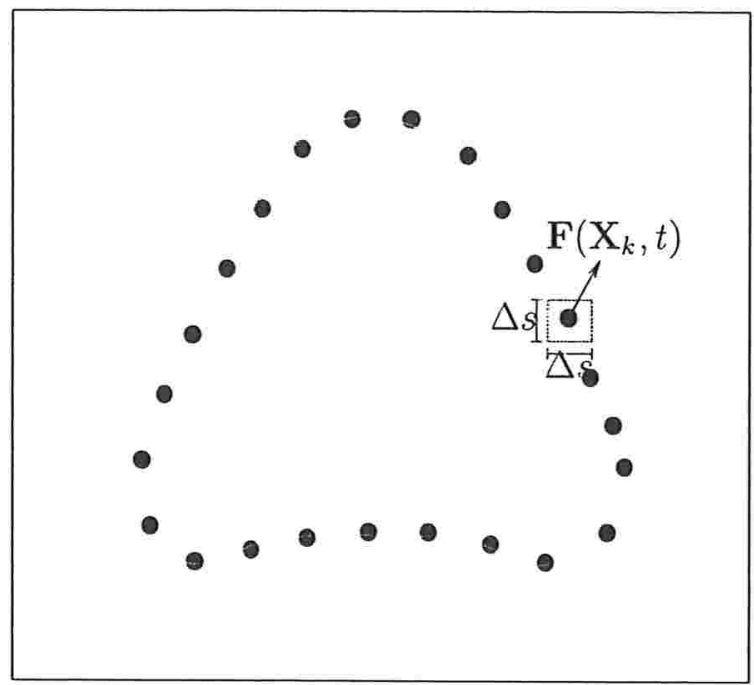

$\Omega$

Figura 2.5: Volume de controle ao redor do ponto lagrangiano $\mathrm{X}_{k}$ para a aplicação do balanço da quantidade de movimento.

onde:

$$
\begin{aligned}
\mathbf{F}_{\mathbf{a}} & =\rho \frac{\partial \mathbf{u}}{\partial t} \\
\mathbf{G} & =\rho(\mathbf{u} \cdot \nabla \mathbf{u})+\nabla p-\nabla \cdot(2 \mu \mathbf{d})
\end{aligned}
$$

são denominadas força de aceleração e força complementar, respectivamente.

O cálculo da força de aceleração foi feito mediante a seguinte aproximação:

$$
\mathbf{F}_{\mathbf{a}}^{n+1}\left(\mathbf{X}_{k}^{n+1}\right)=\rho\left(\frac{\mathbf{V}^{n+1}-\mathbf{u}_{f k}^{n}}{\Delta t}\right)
$$

onde $\mathrm{V}^{n+1}$ é a velocidade da estrutura no tempo $t^{n+1}$ e $\mathbf{u}_{f k}^{n}$ é a velocidade do fluido no tempo $t_{n}$ no ponto lagrangiano $\mathrm{X}_{k}^{n+1}$. Para determinar o valor de $\mathrm{G}$ dado pela equação (2.28), um processo de interpolação é aplicado.

Lima e Silva et al. [25] apresentaram simulações dentro do contexto do método de diferenças finitas. Um processo para o cálculo do termo $\mathrm{G}$ é dado. O escoamento de um fluido ao redor de um cilindro estacionário é apresentado, entre outros. A boa concordância dos valores dos coeficientes de arrasto e sustentação e número de Strouhal foram reportados. 
Outros trabalhos em duas dimensões para corpos com velocidade prescrita não nula foram apresentados por: Arruda [1], escoamento induzido em um conjunto canal-cavidade com fundo móvel periódico; Silva et al. [41], escoamento sobre cilindros circulares oscilando ou girando em número de Reynolds acima de 1000, e Oliveira et al. [32], escoamento ao redor de um cilindro de diâmetro variável no tempo. Os resultados apresentaram boa concordância quantitativa e/ou qualitativa.

No caso da velocidade de estrutura rígida não ser prescrita, uma simulação foi apresentada por Alessandra et al. [49], para a queda livre de uma partícula circular em um fluido viscoso. Comparações com outros resultados apresentaram boa concordância.

\subsubsection{Imposição da presença da interface através de células fan- tasmas}

Nos métodos anteriores, a imposição da condição de contorno devido à presença do corpo é feita através de um campo forçante aplicado ao redor da interface ou através da escolha de pontos de aplicação do termo forçante discreto.

No método baseado em células fantasmas a imposição da condição de contorno se dá através das células fantasmas. Estas células são definidas como células internas ao corpo que tem pelo menos uma célula de fluido vizinha. Na Figura 2.6-(a), mostra-se algumas células fantasmas $(\Delta)$, e células de fluido $(\mathbf{x})$.

Nestas células fantasmas modifica-se o estêncil associado à variável genérica $\phi$ por uma expressão encontrada mediante um processo de extrapolação local. Este processo de extrapolação tem como base uma função obtida mediante o uso do(s) valor(es) da variável(eis) no contorno e em outras células de fluido. É através dessa função que se tem em conta o valor da variável no contorno e permite-se impor indiretamente o valor delas nas equações. Funções lineares, bilineares, quadráticas, etc. podem ser consideradas em Majumdar et al [26].

Por exemplo, para o ponto G da Figura 2.6-(b) mostra-se um conjunto de pontos do fluido $\left(\mathrm{X}_{1}, \mathrm{X}_{2} \ldots \mathrm{X}_{5}\right)$ e um ponto do contorno $\mathrm{O}$, que podem ser usados para definir uma função para a variável $\phi$. Para o caso de se considerar, somente, os pontos $\mathrm{O}, \mathrm{X}_{1}$, e $\mathrm{X}_{2}$ pode-se determinar a função linear:

$$
\phi=a_{0}+a_{1} x+a_{2} y
$$

onde $a_{0}, a_{1}$ e $a_{2}$ são coeficientes obtidos através dos valores da função $\phi$ nos três pontos 


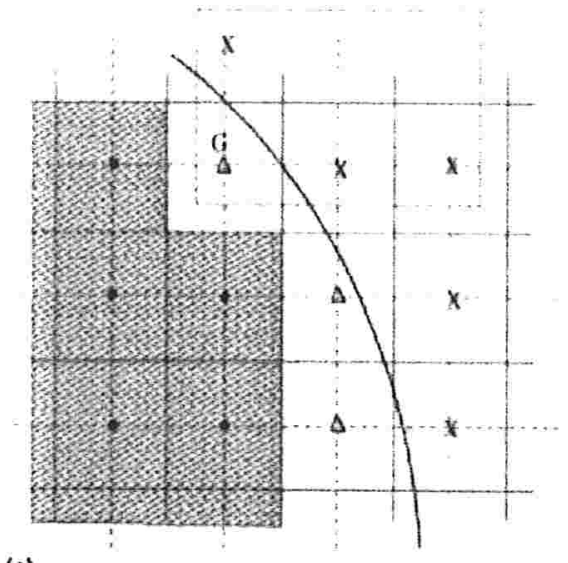

(a)

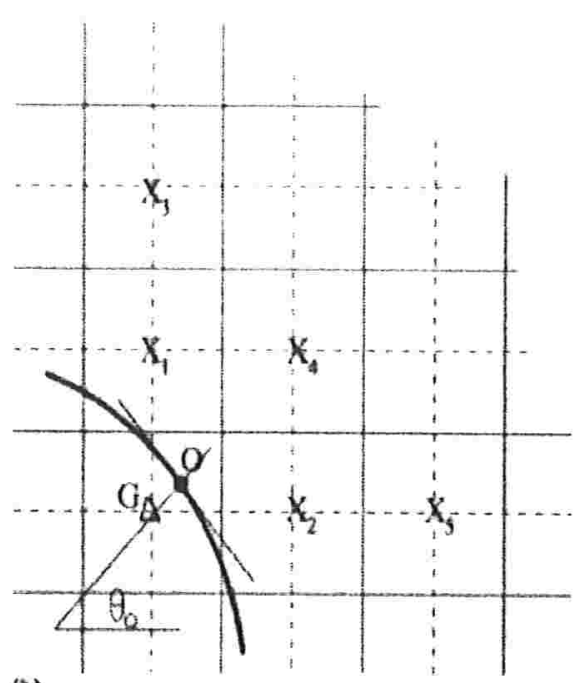

(b)

Figura 2.6: (a) Exemplo de um domínio computacional com uma fronteira imersa, x, ponto no domínio físico e $\Delta$, células fantasmas do domínio; (b) esquema de pontos usados para avaliar a variável localizada na células fantasma G [52].

especificados acima.

Com base nesta função, o valor de $\phi$ no ponto $G$ pode ser expresso como

$$
\phi=\alpha_{0} \phi_{0}+\alpha_{1} \phi_{1}+\alpha_{2} \phi_{2}
$$

onde os coeficientes $\alpha_{0} \ldots \alpha_{2}$ são constantes determinadas no processo de avaliação de $\phi$ no ponto $\mathrm{G}$ e $\phi_{0}, \phi_{1}$ e $\phi_{2}$ são os valores da variável $\phi$ nos pontos $\mathrm{O}, \mathrm{X}_{1}$ e $\mathrm{X}_{2}$, respectivamente.

Entre as aplicações desta metodologia, ou variantes dela, tem-se: escoamento ao redor de um road-vehicle [48], investigação numérica e experimental do escoamento turbulento em serpentinas com chicanas [18], Solvers RANS com malhas estruturadas adaptativas não conformes à interface [26].

\subsubsection{Imposição da presença da interface através da reconstrução das células cortadas pela interface}

Uma outra metodologia, baseada em volumes finitos, foi apresentada por Ye et al [51]. Ela consiste em redefinir as células que são cortadas pela interface (considerada como 
polinômio linear por partes) e calcular os fluxos necessários para o cálculo das integrais nesses volumens. A forma de redefinir as células é tal que a nova célula tenha a interface como contorno da célula e, assim, imposição da condição de contorno devido à interface é satisfeita.

A proposta de Ye et al. [51] foi definir células trapezoidais para as células cortadas pela interface. Uma vez identificadas as células cortadas pela interface e os pontos onde elas são cortadas, verifica-se se o centro da célula está no fluido. Se for assim, elimina-se a porção da célula que está no sólido e adicionam-se as partes das outras células cujos centros estão no caem no fluido. As figuras 2.7-(a),(b) mostram exemplos da redefinição de células cortadas pela interface. Dependendo da localização e da orientação local da interface, células trapezoidais de diferentes dimensões podem ser formadas.

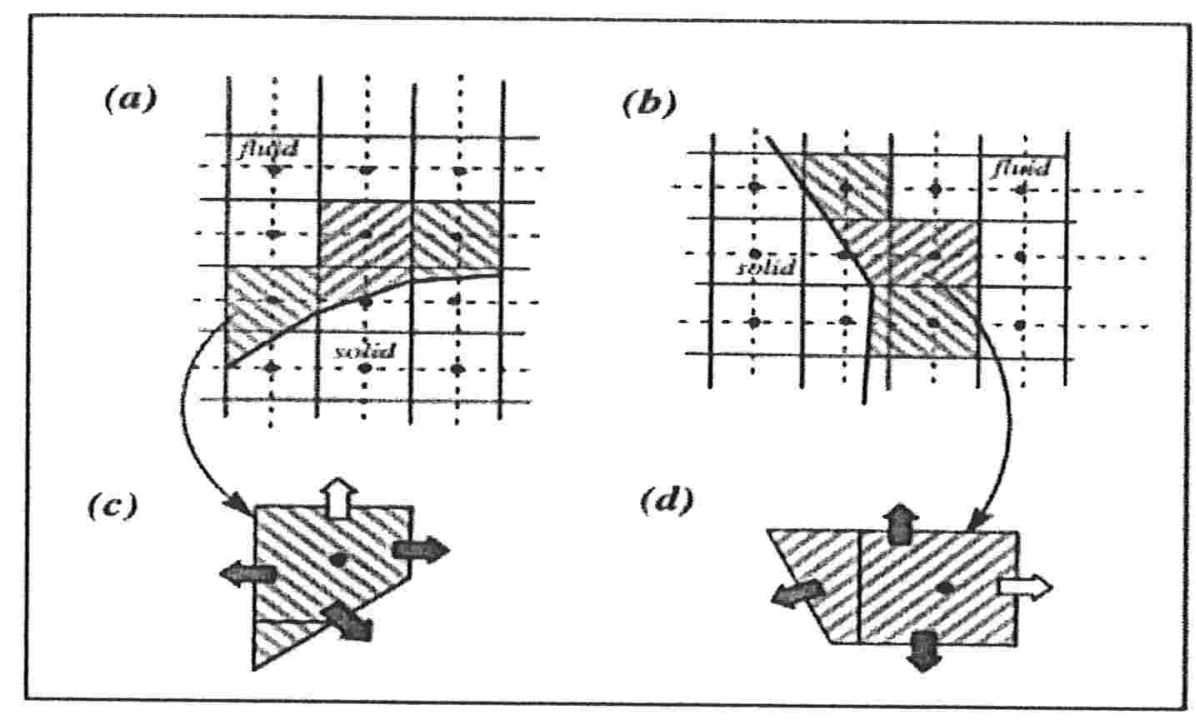

Figura 2.7: Exemplos de células trapezoidais ao redor da interface imersa [51].

Na Figura 2.7-(c),(d), mostram-se duas células trapezoidais. Os fluxos devem ser determinados nos lados destas células. Ye et al. [51] propuseram definir uma função para a variável genérica $\phi$ nos lados das células trapezoidais, com base no seu valor dela mesma em outros pontos conhecidos e, com ela, determinar os fluxos necessários. Por exemplo, para a célula trapezoidal mostrada na Figura 2.8-(a), uma função é definida para o cálculo do fluxo em $f_{s_{w}}$. Esta função é produto da interpolação da variável $\phi$ em seis pontos ( pontos do fluido (1),(2), (3) e (4) e pontos do contorno (5) e (6)), cuja expressão é a 


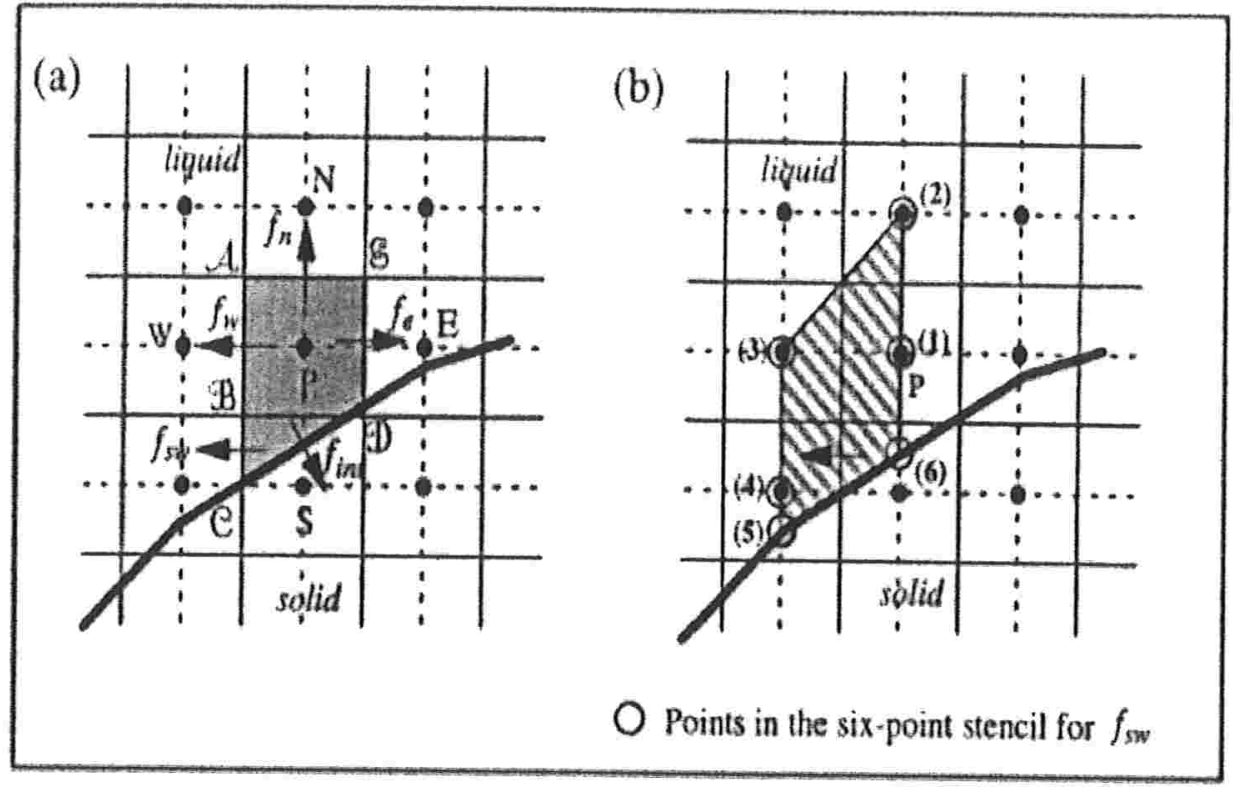

Figura 2.8: Exemplo do estêncil usado para interpolar os fluxos nos lados do trapézio [51].

seguinte:

$$
\phi=c_{1} x y^{2}+c_{2} y^{2}+c_{3} x y+c_{4} x+c_{5} y+c_{6} .
$$

Os valores dos coeficientes $c_{i}$ 's $i=1: 6$ são determinados pelos valores de $\phi$ nos seis pontos indicados. Baseado nessa função, serão determinados os fluxos necessários no ponto $f_{s_{w}}$.

Exemplos da aplicação em duas dimensões foram apresentadas por Ye et al. [51]. Outros exemplos da aplicação desta metodologia são: objetos em queda livre [29], entre outros.

\subsubsection{Imposição da presença da interface através de um termo do tipo multiplicador de Lagrange}

Glowinski et al. [13] apresentaram uma técnica denominada Método de Domínio Fictício, no qual considera-se o fluido ocupando um domínio maior do que o ocupado por ele mesmo. Além disso, um termo do tipo multiplicador de Lagrange é acrescentado nas equações do fluido para simular a presença da estrutura. A discretização foi baseada em elementos finitos e uma única equação variacional foi usada para o sistema fluido-sólido (evitando-se 
o cálculo das forças hidrodinâmicas e dos torques). Eles apresentaram uma simulação de duas partículas em um canal bidimensional, 504 partículas numa caixa bidimensional e a interação de duas partículas esféricas em 3D.

Juárez et al. [20] usaram o método de domínio fictício para simular numericamente a dinâmica de um pêndulo imerso em um fluido. No mesmo trabalho apresentaram a simulação numérica de dois pêndulos imersos em um fluido fixos no mesmo ponto mas soltos de diferentes pontos iniciais. Resultados mostraram comportamentos qualitativos esperados.

\subsection{Resumo sobre as metodologias baseadas no MFI}

Nesta seção, apresenta-se um resumo das características, vantagens e desvantagens das metodologias baseadas do Método da Fronteira Imersa apresentados na seção anterior

\section{Termo forçante de Peskin [34]}

Características e desvantagens:

- Requer uso de constantes a serem ajustadas para representar a rígidez do corpo, o que implica o uso de pequenos passos no tempo

- Uso de uma função de distribuição suave para o delta de Dirac e isto implica na representação enlargecida da fronteira imersa

- Convergência de primeira ordem

Características e vantagens:

- Independência do termo forçante com a discretização espacial

- O cálculo das forças fluidodinâmicas e torque é direto.

2. Termo forçante de Goldstein et al. [14]

Características e desvantagens:

- Requer uso de constantes a serem ajustadas para representar a rígidez do corpo e a sua implicação em pequenos passo no tempo

- Uso de uma função de distribuição suave para o delta de Dirac e a implicação na representação enlargecida da fronteira imersa 
Características e vantagens:

- Independência do termo forçante com a discretização espacial

- O cálculo das forças fluidodinâmicas e torque é direto

3. Termo forçante de Mohd-Yusof [30]

Características e desvantagens:

- Necessidade da escolha dos pontos de aplicação do termo forçante

- Necessidade de esquemas de interpolação para determinar a velocidade a ser usada nos pontos de aplicação do termo forçante

- O cálculo da força fluidodinâmica e torque não é tão direto, pois depende da condição do movimento e geometria do corpos

Características e vantagens:

- Não requer constantes a serem ajustada para impor a rígidez do corpo. Passo no tempo restrito pelo método numérico usado

- Independência do termo forçante com a discretização espacial

4. Termo forçante de Lima e Silva et al. [25]

Características e desvantagens:

- Uso de uma função de distribuição suave para o delta de Dirac e a implicação na representação enlargecida da fronteira imersa

- Convergência de primeira ordem

Características e vantagens:

- Não requer o uso de constantes a serem ajustadas para representar a rígidez do corpo. Em principio, o passo no tempo está restrito pelo método usado para resolvê-las

- Independência do termo forçante com a discretização espacial

- O cálculo das forças fluidodinâmicas e torque é direto

5. Imposição da presença da interface através de células fantasmas

Características e desvantagens: 
- Necessidade de processos de interpolação para determinar o estêncil associado aos pontos fantasmas que conservem a ordem de convergência do método

- Aplicação de métodos eficientes para a resolução dos sistemas lineares pode sofrer baixa na ordem de convergência, quando aplicados para as novas equações modificadas

Características e vantagens:

- Não requer um termo forçante e sim de uma modificação do estêncil para os pontos fantasmas

- Representação da interface não é modificada

6. Imposição da presença da interface através da reconstrução das células cortadas pela interface

Características e desvantagens:

- Necessidade de processos de modificação das células computacionais cortadas pela interface em células trapezoidais. Dependendo da localização e orientação local da interface, células trapezoidais de diferentes dimensões podem ser formadas

- Problemas de generalização para simulações em três dimensões, devido à dificuldade da determinação das células cortadas pela interface

- Necessidade de processo de interpolação para a determinação das funções a serem usadas no cálculo do fluxo

- Necessidade da condição de contorno para a pressão na interface

Características e vantagens:

- Representação da interface não é modificada

- Melhor representação da condição de contorno, por considerar células ao redor da interface que conforma ao corpo

- Melhores propriedades de conservação da massa e quantidade de movimento ao redor do contorno 
Cada um dos métodos da fronteira imersa descritos nesta revisão bibliográfica apresentam vantagens e desvantagens. As simulações computacionais desenvolvidas pelos autores com estes métodos para uma variedade de problemas mostram a sua aplicabilidade. Algumas simulações são para problemas de interação parcial, isto é, estrutura rígida determina a dinâmica do fluido, outras para problemas de interação total, isto é, a dinâmica do sistema fluido-estrutura é determinada por ambos.

Neste trabalho, emprega-se o MFI/MFV com o intuito de estudar problemas de interação total. O modelo matemático que usa esta metodologia é apresentado no capítulo a seguir. 


\section{Modelagem matemática}

Neste Capítulo, apresentam-se as equações do modelo matemático adotado para estudar a interação fluido-estrutura.

$\mathrm{Na}$ Seção 3.1, são apresentadas as equações do modelo matemático para o sistema fluido-estrutura mediante o Modelo Físico Virtual (MFV) ([25]). Um elemento importante usado no cálculo da força lagrangiana do MFV é a função indicadora, apresentada na na Seção 3.2. Altos números de Reynolds podem acontecer no processo de interação fluidoestrutura gerando turbulência no escoamento. Para modelar a turbulência é usada a metodologia baseada na Simulação das Grandes Escalas a qual é apresentada na Seção 3.3 .

\subsection{Modelo Físico Virtual}

O Modelo Físico Virtual, proposto por Lima e Silva et al. [25] para modelar interações fluido-estrutura é utilizado dentro da metodologia da fronteira imersa (Seção 2.3). Tal método está baseado na imposição de um termo forçante nas equações do fluido para modelar a presença da estrutura.

As equações do fluido terão a seguinte forma:

$$
\begin{aligned}
\rho\left(\frac{\partial \mathbf{u}}{\partial t}+\mathbf{u} \cdot \nabla \mathbf{u}\right) & =-\nabla p+\nabla \cdot\left\{\mu\left(\nabla \mathbf{u}+\nabla \mathbf{u}^{t}\right)\right\}+\mathbf{f}, \\
\nabla \cdot \mathbf{u} & =0 .
\end{aligned}
$$

onde a expressão para o termo forçante, $\mathbf{f}$, depende do termo fonte $\mathbf{F}$, definido na interface, isto é,

$$
\mathbf{f}=\mathbf{f}(\mathbf{F}) .
$$


Os termos forçantes $\mathbf{f}$ e $\mathbf{F}$ são denominados, aqui, força por unidade de volume euleriana e lagrangiana, respectivamente.

As equações (3.1)-(3.2) são usadas por outras metodologias baseadas no Método da Fronteira Imersa (Peskin [34], Goldstein et al. [14]). A diferença entre eles está na expressão dada para as densidades de forças $\mathbf{f}$ e $\mathbf{F}$.

A densidade de força lagrangiana no MFV é deduzida usando-se o balanço da quantidade de movimento nos pontos da interface $\mathbf{X}_{k}$. Esta densidade de força lagrangiana tem a seguinte expressão:

$$
\mathbf{F}\left(\mathbf{X}_{k}, t\right)=\mathbf{F}_{a}\left(\mathbf{X}_{k}, t\right)+\mathbf{F}_{i}\left(\mathbf{X}_{k}, t\right)+\mathbf{F}_{p}\left(\mathbf{X}_{k}, t\right)+\mathbf{F}_{d}\left(\mathbf{X}_{k}, t\right),
$$

onde:

$$
\begin{aligned}
\mathbf{F}_{a} & =\rho \frac{\partial \mathbf{u}}{\partial t} \\
\mathbf{F}_{\boldsymbol{i}} & =\rho(\mathbf{u} \cdot \nabla \mathbf{u}), \\
\mathbf{F}_{p} & =\nabla p \\
\mathbf{F}_{d} & =-\nabla \cdot(2 \mu \mathbf{d}),
\end{aligned}
$$

e são chamados de força de aceleração, força de inercia, força de pressão e força viscosa.

A expressão (3.4), pode ser escrita da seguinte forma:

$$
\mathbf{F}\left(\mathrm{X}_{k}, t\right)=\mathbf{F}_{a}\left(\mathrm{X}_{k}, t\right)+\mathbf{G}\left(\mathrm{X}_{k}, t\right)
$$

com:

$$
\mathbf{G}=\mathbf{F}_{i}+\mathbf{F}_{p}+\mathbf{F}_{d}
$$

denominada aqui de força complementar. Para determinar a força lagrangiana $\mathbf{F}$ deve-se conhecer a força de aceleração e a força complementar.

Dependendo do método usado para resolver as equações do fluido no tempo e no espaço uma expressão para o termo densidade de força lagrangiana será dada. Por exemplo, no caso de usar um método de Euler explícito e no caso da velocidade da estrutura ser prescrita, a força de aceleração pode ser aproximada por:

$$
\mathbf{F}_{a}\left(\mathbf{X}_{k}^{n+1}, t^{n}\right)=\rho \frac{\mathbf{u}^{n+1}\left(\mathbf{X}_{k}^{n+1}\right)-\mathbf{u}^{n}\left(\mathbf{X}_{k}^{n+1}\right)}{\Delta t},
$$

onde: $\mathrm{X}_{k}^{n+1}$ é a posição da estrutura no tempo $t^{n+1}, \mathrm{u}^{n+1}\left(\mathrm{X}_{k}^{n+1}\right)$ é a velocidade do fluido no tempo $t^{n+1}$ na posição $\mathbf{X}_{k}^{n+1}, \mathbf{u}^{n}\left(\mathrm{X}_{k}^{n+1}\right)$ é a velocidade do fluido no tempo $n$ na posição $\mathrm{X}_{k}^{n+1}$. 
Lima e Silva et al. [25] propuseram usar para as velocidades que aparecem na força de aceleração, equação (3.11), as seguintes relações:

$$
\begin{aligned}
\mathrm{u}^{n+1}\left(\mathrm{X}_{k}^{n+1}\right) & =\mathrm{V}_{k}^{n+1}, \\
\mathrm{u}^{n}\left(\mathrm{X}_{k}^{n+1}\right) & =\mathrm{u}_{f k}^{n} .
\end{aligned}
$$

onde: $\mathrm{V}_{k}^{n+1}$ é a velocidade da interface no tempo $t^{n+1}$ e $\mathrm{u}_{f k}^{n}$ é a velocidade do fluido no tempo $t^{n}$ e no ponto $\mathrm{X}_{k}^{n+1}$. Esta última é calculada através de um processo de interpolação e ser explicado abaixo. Logo, a força de aceleração terá seguinte expressão [25]:

$$
\mathbf{F}_{a}\left(\mathbf{X}_{k}^{n+1}, t_{n}\right)=\rho \frac{\mathbf{V}_{k}^{n+1}-\mathbf{u}_{f k}^{n}}{\Delta t} .
$$

A expressão para o termo $\mathbf{G}$, equação (3.10), tem derivadas em x e y da velocidade e pressão avaliadas nos pontos da interface. Para o cálculo dessas derivadas os autores propuseram determinar polinômios de segundo grau para $u, v$ e $p$ nas direç̧ões das componentes do vetor normal à interface, e usar as derivadas no espaço destes polinômios para aproximar as respectivas derivadas que compõem o termo $\mathbf{G}$.

Para determinar os polinômios para $u, v$ e $p$ na direção $x$ e $y$, pontos auxiliares são usados. Os pontos adicionais em $x$ foram denotados por $\mathrm{x}_{1}$ e $\mathrm{x}_{2}$ distantes $\Delta x$ e $2 \Delta x$ de $\mathrm{X}_{k}$, respectivamente e os pontos em y são denotados por $\mathrm{x}_{3}$ e $\mathrm{x}_{4}$ e distam $\Delta y$ e $2 \Delta y$ de $\mathrm{X}_{k}$, respectivamente (Figura 3.1). As velocidades e pressões nesses pontos adicionais são denotadas por $\left(u_{i}, v_{i}\right)$ e $p_{i}, i=1,2,3,4$ e estas devem ser interpoladas das velocidades e pressões do fluido. $\mathrm{Em} \mathrm{X}_{k}$, determina-se a velocidade do fluido $\mathbf{u}_{f k}=\left(u_{f k}, v_{f k}\right)$ e a pressão $p_{k}$, por um processo de interpolação similar ao adotado para os pontos auxiliares e, determina-se a velocidade da interface (no caso dela não ser conhecida) através das equações associada à estrutura.

O processo de interpolação adotado para o cálculo de $\left(u_{i}, v_{i}\right) i=1,2,3,4\left(\mathrm{e}\left(u_{f k}, v_{f k}\right)\right)$ é dado por:

$$
\begin{aligned}
u_{i} & =\int_{\Omega} u(\mathbf{x}) \delta\left(\mathbf{x}-\mathbf{x}_{i}\right) \Delta x \Delta y \\
v_{i} & =\int_{\Omega} v(\mathbf{x}) \delta\left(\mathbf{x}-\mathbf{x}_{i}\right) \Delta x \Delta y
\end{aligned}
$$

onde: $\delta$ é uma aproximação do delta de Dirac. Aqui, a seguinte aproximação para o delta de Dirac é usada

$$
\delta\left(\mathrm{x}-\mathrm{x}_{i}\right)=\frac{D\left(\frac{x-x_{i}}{\Delta x}\right) D\left(\frac{y-y_{i}}{\Delta y}\right)}{\Delta x \Delta y},
$$




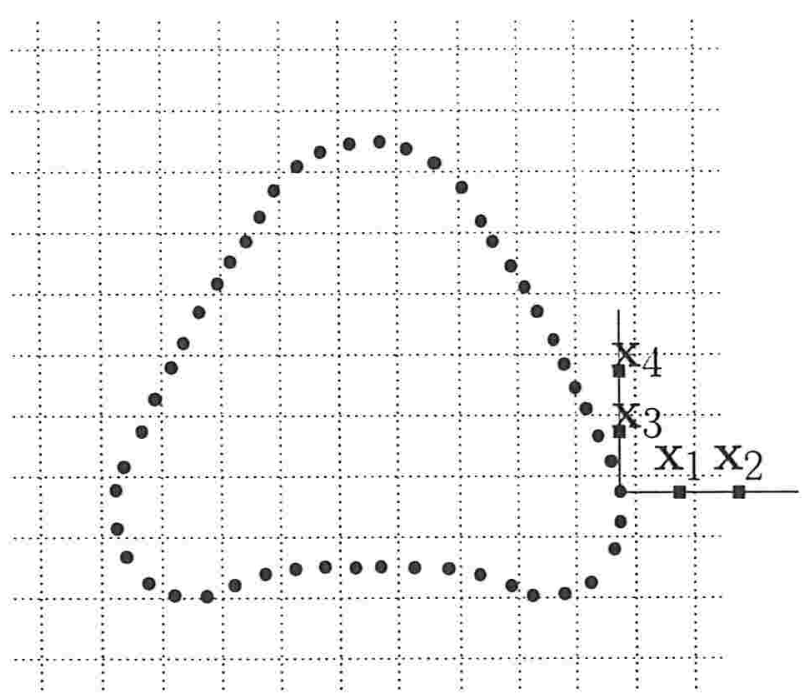

Figura 3.1: Esquema para a interpolação da pressão e da velocidade.

onde a função unidimensional $D$ representa um peso da contribuição do valor da variável em $\mathrm{x}$ no ponto $\mathrm{x}_{i}$ dependendo da distância relativa $\frac{\left|x-x_{i}\right|}{\Delta x}$ (ou $\frac{\left|y-y_{i}\right|}{\Delta y}$ ) e suporte da função $D$, neste caso $[-2,0,2,0]$, função (2.18).

Para o cálculo da pressão $p_{i}, i=1, \ldots 4$ (e $p_{k}$ ) é usada a expressão:

$$
p_{i}=\frac{\int_{\Omega} p(\mathbf{x}) \delta\left(\mathbf{x}-\mathbf{x}_{i}\right)(1-I(\mathbf{x})) \Delta x \Delta y}{\int_{\Omega} \delta\left(\mathbf{x}-\mathbf{x}_{i}\right)(1-I(\mathrm{x})) \Delta x \Delta y},
$$

onde a função $I(\mathrm{x})$ é denominada função indicadora (Seção 3.2) cujo valor é 1 se $\mathrm{x}$ é um ponto dentro do corpo e é zero se o ponto estiver fora do corpo.

Uma vez determinada a densidade de força lagrangiana, F, esta deve ser espalhada para os pontos da malha euleriana e assim determinar $f$, que está encargada de impor a presença da interface no fluido.

Lima e Silva et al. [25] usaram um processo de espalhamento da força lagrangiana em pontos da malha euleriana que distam duas células do respectivo ponto lagrangiano. Para pontos mais distantes, a contribuição da força lagrangiana é considerada nula. Vários pontos lagrangianos podem contribuir na força euleriana. O número de pontos lagrangianos pode ser identificado mediante a determinação do número de pontos lagrangianos que estão dentro de um retângulo de dimensões $4 \Delta x \times 4 \Delta y$ com centro no ponto euleriano. Na Figura 3.2, ilustra-se o número de pontos lagrangianos que influenciam na força euleriana em $\mathrm{x}_{a}$ e $\mathrm{x}_{b}$. Para o ponto $\mathrm{x}_{a}$ o retângulo contem três pontos lagrangianos que determi- 
nam a força nesse ponto, enquanto que para o ponto $\mathrm{x}_{b}$, no retângulo correspondente, seis pontos lagrangianos estão contidos e esses determinam a força em tal ponto euleriano.

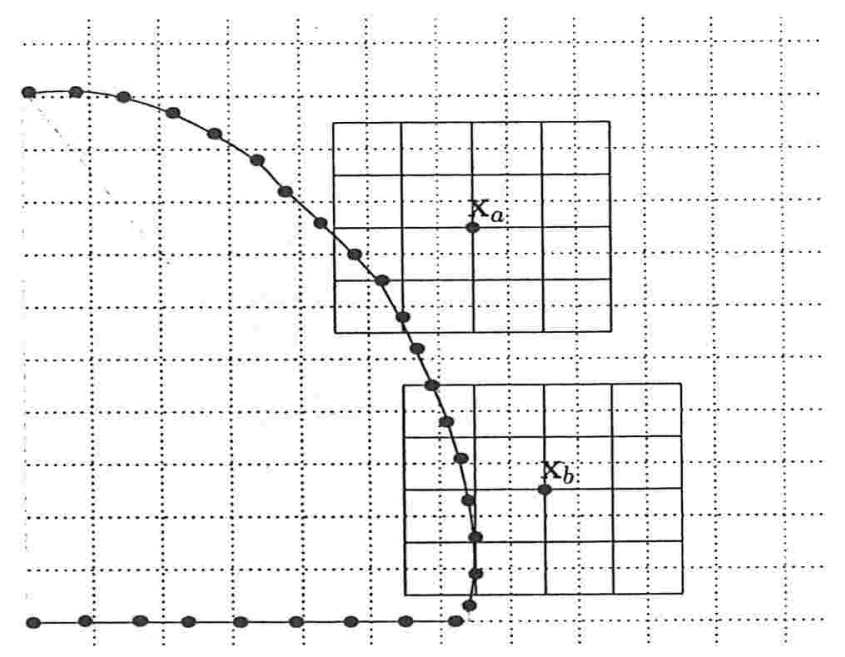

Figura 3.2: Exemplo da influência da contribuição de pontos forçantes lagrangianos em pontos eulerianos.

A determinação da expressão para $\mathbf{f}=\mathbf{f}(\mathbf{F})$ é baseada nas seguintes considerações:

- as densidades de força euleriana e lagrangiana possuem a mesma dimensão, isto é, força por unidade de volume;

- os volumes de controle usados para os pontos eulerianos e lagrangianos são $\Delta V=$ $\Delta x \Delta y \Delta z$ e $\Delta V_{k}=\Delta s_{k} \Delta s_{k} \Delta z$, respectivamente;

- o peso da contribuição da força lagrangiana é dado com base na função $D$ com suporte $[-2,0,2,0]$.

Baseamdo-se nessas três condições, a força no volume do fluido $\Delta V$ com centro no ponto euleriano $\mathbf{x}_{i j}$ é dada por:

$$
\mathbf{f}_{i j} \Delta V=\sum_{k=1}^{N p l} \mathbf{F}_{k} \Delta V_{k} D\left(\frac{x_{i j}-X_{k}}{\Delta x}\right) D\left(\frac{y_{i j}-Y_{k}}{\Delta y}\right),
$$

e portanto:

$$
\mathrm{f}_{i j}=\sum_{k=1}^{N p l} \mathrm{~F}_{k} \Delta s_{k} \Delta s_{k} \frac{D\left(\frac{x_{i j}-X_{k}}{\Delta x}\right) D\left(\frac{y_{i j}-Y_{k}}{\Delta y}\right)}{\Delta x \Delta y}
$$


onde $N p l$ é o número total de pontos lagrangianos. O fator $\frac{D\left(\frac{x_{i j}-X_{k}}{\Delta x}\right) D\left(\frac{y_{i j}-Y_{k}}{\Delta y}\right)}{\Delta x \Delta y}$ é uma aproximação suave para a função delta de Dirac. Portanto a equação (3.15) pode ser expressa como

$$
\mathbf{f}_{i j}=\sum_{k=1}^{N p l} \mathbf{F}_{k} \delta\left(\mathbf{x}_{i j}-\mathbf{X}_{k}\right) \Delta s_{k} \Delta s_{k}
$$

Esta força euleriana é a utilizada para impor a presença da estrutura imersa no fluido.

Para a determinação da dinâmica do corpo rígido são usadas as equações de NewtonEuler, isto é,

$$
\begin{aligned}
M_{c} \frac{d \mathbf{V}_{c}}{d t} & =\mathbf{G}_{c}+\mathbf{F}_{f c} \\
\mathbf{I}_{c} \frac{d}{d t}(\mathbf{w}) & =\mathbf{T}_{f c} \\
\frac{d \mathbf{X}_{c}}{d t} & =\mathbf{V}_{c} \\
\frac{d \theta_{\mathbf{c}}}{d t} & =w
\end{aligned}
$$

onde $M_{c}$ e $\mathrm{I}_{c}$ são a massa e o momento de inércia da estrutura; $\mathrm{V}_{c}$ e $w$ são a velocidade de translação do CM e a velocidade angular do corpo; $\mathrm{X}_{c}$ e $\theta_{c}$ são a posição do CM e o delocamento angular; $\mathbf{G}_{c}$ é o peso do corpo; $\mathbf{F}_{f c}$ e $\mathbf{T}_{f c}$ são a força hidrodinâmica total atuando no corpo e o torque total no corpo devido às forças hidrodinâmicas, respetivamente.

O cálculo da força fluidodinâmica total é determinado pela soma de todas as forças que o fluido exerce no contorno da estrutura. No MFV a força de ação do fluido na estrutura pode ser determinada em função da força lagrangiana e da terceira lei de Newton, segundo o qual a toda ação corresponde uma reação de igual magnitude mas em sentido contrário. A força de ação da estrutura no fluido é dado por $\mathbf{F}_{k} \Delta s_{k}^{2}$, portanto a força de reação do fluido na estrutura é dado por $-\mathbf{F}_{k} \Delta s_{k}^{2}$. Baseado nisto, as expressões para $\mathbf{F}_{f c}$ e $\mathbf{T}_{f c}$ são:

$$
\begin{aligned}
\mathbf{F}_{f c} & =\sum_{k=1}^{N p l}\left(-\mathbf{F}_{k} \Delta s_{k} \Delta s_{k}\right) \\
\mathbf{T}_{f c} & =\sum_{k=1}^{N p l}\left(\mathbf{X}_{k}-\mathbf{X}_{c}\right) \times\left(-\mathbf{F}_{k} \Delta s_{k} \Delta s_{k}\right)
\end{aligned}
$$




\subsection{Função indicadora}

A determinação da região ocupada pelas componentes de um sistema fluido-estrutura rígida ou fluido-fluido é importante para identificação das propriedadès físicas no domínio euleriano ou para os elementos que o processo de solução da metodologia requer.

A seguir, é apresentada uma forma de definir uma função denominda função indicadora, que determina a região ocupada pelos elementos que compõem o sistema de estudo fluido-estrutura ou fluido-fluido. O cálculo desta função é baseada nas propriedades geométricas da interface, definidas pelas componentes do sistema.

\section{Função indicadora de Unverdi e Tryggvason}

Unverdi e Tryggvason [45] propuseram um método para o cálculo das propriedades físicas dos fluidos, que se baseia em uma função indicadora. Este método pode ser usado para determinar uma função que indique a região ocupada por uma estrutura rígida imersa em um fluido. Neste caso, a estrutura imersa é denominada fase dispersa e o fluido fase contínua.

A função indicadora de Unverdi e Tryggavason, $I(\mathrm{x}, t)$, é calculada por um termo fonte $\mathrm{G}(\mathrm{x}, t)$, o qual é definido por:

$$
\nabla I(\mathrm{x}, t)=\mathrm{G}(\mathrm{x}, t)
$$

sendo $\mathrm{G}(\mathrm{x}, t)$ :

$$
\mathbf{G}(\mathbf{x}, t)=\sum_{k} D\left(\mathbf{x}-\mathbf{X}_{k}\right) \mathbf{n}\left(\mathbf{X}_{k}\right) \Delta s\left(\mathbf{X}_{k}\right),
$$

com $D\left(\mathbf{x}-\mathbf{X}_{k}\right)$ uma função de distribuição, $\mathbf{n}\left(\mathbf{X}_{k}\right)$ o vetor normal à interface e $\Delta s\left(\mathbf{X}_{k}\right)$ a distância entre dois pontos lagrangianos consecutivos.

Aplicando o operador divergente à equação (3.23), obtém-se

$$
\nabla^{2} I(\mathrm{x}, t)=\nabla \cdot \mathrm{G}(\mathrm{x}, t)
$$

que é uma equação de Poisson a ser resolvida para a obtenção de $I(\mathrm{x}, t)$.

A expressão para $\mathrm{G}$, equação (3.24), é definida de tal forma que a fase dispersa tome o valor de um (estrutura rígida) e a fase contínua tome valor zero (fluido). O uso da função de distribuição, $D$ na expressão para $\mathrm{G}$, determina que a interface seja representada por uma interface enlarguecida. Nesta região, o valor da função indicadora assume valores entre zero e um. As considerações acima determinam que a condição de contorno para a equação (3.25) é a condição de Dirichlet homogênea, isto é, $\left.I\right|_{\partial \Omega}=0$. 


\subsection{Modelagem da turbulência: SGE}

Os escoamentos turbulentos são caracterizados por um elevado número de graus de liberdade e uma ampla gama de estruturas turbillonares tridimensionais. Essas estruturas, em função de certas características, compoem uma banda de escalas que vão desde as grandes escalas até as pequenas escalas dissipativas de Kolmogorov.

Uma forma de capturar todas as estruturas do escoamento do fluido é mediante a solução das equações do fluido em malhas muito finas. Este processo de solução é denominada de Simulação Numérica Direta (SND), quando todas as escalas da turbulência são resolvidas.

Devido ao custo computacional que a SND carrega, são procuradas outras metodologias para simular escoamentos turbulentos. Uma revisão destas metodologias podem ser encontrada em Silveira-Neto [42].

Neste trabalho, a metodologia a ser empregada para simular a turbulência nos fluidos é baseada na solução das estruturas de grande escala e na modelagem das pequenas escalas, que é conhecida como Simulação de Grandes Escalas (SGE). Em SGE, são resolvidas as estruturas de grandes escalas e modeladas as estruturas de pequenas escalas. A separação de escalas é possível, sob a suposição que as pequenas escalas são aproximadamente isotrópicas e independentes da geometria e da condição de contorno, enquanto que as grandes escalas são mais anisotrópicas e variam de um escoamento para outro.

A separação das escalas é feita atráves de um processo de filtragem. Mediante o processo de filtragem, uma variável $\phi$ pode ser expressa por

$$
\phi=\bar{\phi}+\phi^{\prime}
$$

onde $\bar{\phi}$ é a variável filtrada correspondente à estrutura de grande escala e $\phi^{\prime}$ é a variável corresponde à estrutura de pequena escala. A expressão para determinar $\bar{\phi}$ é dada por (caso unidimensional)

$$
\bar{\phi}(x)=\int G\left(x, x^{\prime}\right) \phi\left(x^{\prime}\right) d x^{\prime},
$$

onde a função $G\left(x, x^{\prime}\right)$ é conhecida com o núcleo do filtro. Núcleos de filtro aplicados em SGE são uma função Gaussiana, uma função filtro caixa, etc. Cada filtro tem associado um comprimento de separação ou de corte, $\Delta$. Escalas de comprimento maiores a $\Delta$ são consideradas como grandes escalas e menores a $\Delta$ correspondem as estruturas de pequenas escalas, também conhecidas como escalas sub-malha.

No processo de filtragem das equações do fluido, surgem tensores adicionais, além dos 
tensores viscosos, os quais devem ser modelados. A seguir, apresentam-se as equações de Naiver-Stokes filtradas em notação de Einstein, para ilustrar o processo de aparecimento de novos termos a serem modelados

$$
\begin{aligned}
\frac{\partial \bar{u}_{i}}{\partial t}+\frac{\partial \overline{u_{i} u_{j}}}{\partial x_{j}} & =-\frac{1}{\rho} \frac{\partial \bar{p}}{\partial x_{i}}+\frac{\partial}{\partial x_{j}}\left(\nu\left[\frac{\partial \overline{u_{i}}}{\partial x_{j}}+\frac{\partial \overline{u_{j}}}{\partial x_{i}}\right]\right), \\
\frac{\partial \bar{u}_{i}}{\partial x_{i}} & =0
\end{aligned}
$$

Observa-se, na equação (3.26), que no termo advectivo aparece o produto filtrado das variáveis de interesse. Para que esta equação possa ser resolvida, as componentes da velocidade que aparecem neste produto serão decompostas. Fazendo a decomposição e aplicando as definições cabíveis, as equações globais da turbulência são:

$$
\begin{aligned}
\frac{\partial \overline{u_{i}}}{\partial t}+\frac{\partial \overline{u_{i}} \overline{u_{j}}}{\partial x_{j}} & =-\frac{1}{\rho} \frac{\partial \bar{p}}{\partial x_{i}}+\frac{\partial}{\partial x_{j}}\left(\nu\left[\frac{\partial \overline{u_{i}}}{\partial x_{j}}+\frac{\partial \overline{u_{j}}}{\partial x_{i}}\right]+\tau_{i j}\right) \\
\overline{\partial u_{i}} & =0
\end{aligned}
$$

onde $\tau_{i j}=-\overline{u_{i}^{\prime} u_{j}^{\prime}}$ é o tensor de Reynolds sub-malha. Detalhes do processo de filtragem acima pode ser encontrado em Silveira-Neto et al. [42] e em Padilla [33]. Conforme se observa na equação (3.28), a consequência imediata do processo de decomposição de escalas e da transformação das equações originais em equações médias é o aparecimento do tensor adicional $\tau_{i j}$, o qual deve ser modelado.

Boussineq propôs um modelo de fechamento para o tensor de Reynolds com o conceito de viscosidade turbulenta. Tal modelo expressa o tensor de Reynolds sub-malha em função da taxa de deformação, gerada pelo campo de velocidade e da energia cinética turbulenta $(k)$ da seguinte forma:

$$
\tau_{i j}=\nu_{t}\left[\frac{\partial \overline{u_{i}}}{\partial x_{j}}+\frac{\partial \overline{u_{j}}}{\partial x_{i}}\right]-\frac{2}{3} k \delta_{i j}=2 \nu_{t} \overline{S_{i j}}-\frac{2}{3} k \delta_{i j}
$$

onde:

$$
\begin{aligned}
k & =\frac{1}{2}\left(\overline{u_{i}^{\prime} u_{j}^{\prime}}\right)=\frac{1}{2}\left(\overline{u^{\prime 2}}+\overline{v^{\prime 2}}+\overline{w^{\prime 2}}\right) \\
\overline{S_{i j}} & =\frac{1}{2}\left(\frac{\partial \overline{u_{i}}}{\partial x_{j}}+\frac{\partial \overline{u_{j}}}{\partial x_{i}}\right)
\end{aligned}
$$

e $\delta_{i j}$ é o delta de Kronecher. 
Substituindo a equação (3.30) na equação (3.28) e incorporando-se a energia cinética ao termo de pressão, obtém-se a seguinte equação:

$$
\frac{\partial \bar{u}_{i}}{\partial t}+\frac{\partial \bar{u}_{i} \overline{u_{j}}}{\partial x_{j}}=-\frac{1}{\rho} \frac{\partial \overline{p^{\prime}}}{\partial x_{i}}+\frac{\partial}{\partial x_{j}}\left(\nu_{e f e}\left[\frac{\partial \overline{u_{i}}}{\partial x_{j}}+\frac{\partial \overline{u_{j}}}{\partial x_{i}}\right]\right)
$$

onde:

$$
\begin{aligned}
\overline{p^{\prime}} & =\bar{p}+\frac{2}{3} k \rho \\
\nu_{\text {efe }} & =\nu+\nu_{t}
\end{aligned}
$$

Observa-se que campo de pressão dada na equação (3.32) é um campo de pressão modificado: pressão filtrada mais uma pressão devido à energia cinética.

A equação (3.31) é a equação de quantidade de movimento para a modelagem de turbulência via a SGE. Tal equação estará completamente determinada se um valor para $\nu_{t}$ é definido.

\subsubsection{Modelagem sub-malha: modelo de Smagorinsky}

A modelagem sub-malha possibilita o cálculo da viscosidade turbulenta via diferentes modelos, buscando representar a idéia de modelar a transferência de energia entre a banda de escalas resolvidas (grandes escalas) e a banda de escalas não resolvidas (pequenas escalas). Aqui considerou-se o modelo sub-malha de Smagorinsky.

\section{Modelo de Smagorinsky}

É um modelo a zero equações de transporte. Simples, mas exige o uso de malhas finas. É deduzido usando-se a hipótese de equilíbrio local para as pequenas escalas, onde a energia injetada no espectro deve ser igual à energia dissipada pelos efeitos viscosos. Assim, a viscosidade turbulenta é função da taxa de deformação $\bar{S}_{i j}$ e do comprimento sub-malha como indica a expressão:

$$
\nu_{t}=\left(C_{s} \Delta\right)^{2}\left\|\bar{S}_{i j}\right\|
$$

onde:

- $C_{s}$ é uma constante empírica, denominada constante de Smagorinsky

- $\Delta$ é o comprimento característico associado ao tamanho da malha e seu o valor é dado por $\left(\Delta_{x} \Delta_{y}\right)^{1 / 2}$

- $\left\|\overline{S_{i j}}\right\|=\sqrt{2 \overline{S_{i j}} \overline{S_{i j}}}$ é o modulo do tensor taxa de deformação do campo filtrado. 
CAPÍtulo 4

\section{Solução numérica da modelagem matemática}

No Capítulo anterior foram apresentadas as equações a serem usadas para modelar a interação fluido-estrutura mediante o Modelo Físico Virtual. Esse conjunto de equações está formado pelas equações de Navier-Stokes com termo forçante (para impor a presença da estrutura) e as equações de Newton-Euler. A solução numérica de tais equações será feita mediante um método de Runge-Kutta explícito de segunda ordem no tempo (RKE2) com discretização espacial de segunda ordem baseada no método de diferenças finitas. Em cada estágio do método de Runge-Kutta, dever-se-á resolver as equações de Navier-Stokes e as equações de Newton-Euler.

A solução numérica das equações do fluido devem levar em conta, entre outras coisas, a não linearidade das equações de Navier-Stokes e a falta de uma equação para a pressão do fluido. Muitas propostas de solução apareceram. Na Seção 4.1, é apresentada uma introdução a duas formas de solução numérica das equações de Navier-Stokes em regime transiente: método de equação de Poisson para a pressão e método de projeção. Método de projeção é um dos métodos mais populares para resolver as equações de Navier-Stokes para escoamentos incompressívieis [24].

Na Seção 4.2 é apresentado o uso do método de projeção para dois métodos explícitos de primeira e segunda ordem no tempo: Runge-Kutta de primeira ordem e de segunda ordem. Apresenta-se também nesta seção um método semi-implícito.

$\mathrm{Na}$ Seção 4.3 são apresentadas as discretizações adotadas, as condições de contorno numéricas e a condição de estabilidade, referentes ao método de solução numérica usado neste trabalho. 
O algoritmo numérico empregado é apresentado na Seção 4.5.

\subsection{Solução das equações de Navier-Stokes: equação de Poisson para a pressão e método de projeção}

As equações de Navier-Stokes para um escoamento incompressível são dadas por:

$$
\begin{aligned}
\rho\left(\frac{\partial \mathbf{u}}{\partial t}+\mathbf{u} \cdot \nabla \mathbf{u}\right) & =-\nabla p+\nabla \cdot(2 \mu \mathbf{d})+\mathbf{f}, \\
\nabla \cdot \mathbf{u} & =0,
\end{aligned}
$$

onde $\mathrm{d}=\frac{\nabla \mathbf{u}+\nabla \mathbf{u}^{T}}{2}$ (tensor taxa de deformação de um fluido).

A condição inicial é dado por: $\mathbf{u}(\mathbf{x}, 0)=\mathbf{u}_{0}$, e a condição de contorno para o campo de velocidade pode ser, entre outras, do tipo Dirichlet ou Neumann.

Tentar desenvolver um esquema numérico para resolver as equações (4.1)-(??) significa lidar com algumas dificuldades destas equações: a não linearidade das equações devido ao termo convectivo, a falta de uma equação para a pressão, etc. A maioria dos esquemas partem de equações discretizadas no tempo. A seguir, apresentam-se algumas aproximações no tempo das equações de Navier-Stokes:

$$
\begin{cases}\rho\left(\frac{\mathbf{u}^{n+1}-\mathbf{u}^{n}}{\Delta t}+(\mathbf{u} \cdot \nabla \mathbf{u})^{n}\right) & =-\nabla p^{n+1}+\nabla \cdot[2 \mu \mathrm{d}]^{n}+\mathrm{f}^{n} \\ \nabla \cdot \mathbf{u}^{n+1} & =0\end{cases}
$$

ou

$$
\begin{cases}\rho\left(\frac{\mathbf{u}^{n+1}-\mathbf{u}^{n}}{d t}+(\mathbf{u} \cdot \nabla \mathbf{u})^{n+1 / 2}\right) & =-\nabla p^{n+1 / 2}+\nabla \cdot[2 \mu \mathrm{d}]^{n+1 / 2}+\mathbf{f}^{n+1 / 2} \\ \nabla \cdot \mathbf{u}^{n+1 / 2} & =0\end{cases}
$$

O sistema (4.3) pode ser obtido integrando-se as equações de Navier-Stokes no intervalo $\left[t^{n}, t^{n+1}\right]\left(\Delta t=t^{n+1}-t^{n}\right)$ [21]. Por outro lado, o sistema (4.4) está baseado numa aproximação centrada no tempo $t^{n+1 / 2}$. Os esquemas (4.3) e (4.4) são de primeira e segunda ordem no tempo.

Uma solução direta do sistema acoplado não é praticável devido à necessidade de métodos robustos para lidar com sistemas não lineares de equações e ao custo computacional que isto acarreta [24]. As metodologias mais populares estão baseadas no desacopla- 
mento do cálculo da velocidade e da pressão, que permite o uso de técnicas mais eficientes para cada um dos processos resultantes.

Harlow e Welch [15] apresentaram o método MAC (marker and cell) desenvolvido para o tratamento de escoamentos incompressíveis com superfícies livres. A proposta para resolver as equações de Navier-Stokes consiste na geração de uma equação de Poisson para a pressão, a partir da equação do balanço da quantidade de movimento discreta no tempo e da condição de livre divergência da velocidade (Sistema 4.3). Uma vez obtida a pressão, o campo de velocidade é calculado da equação de quantidade de movimento considerando a pressão calculada. Vários trabalhos foram desenvolvidos seguindo esta filosofia. E e Liu [50] usaram esta técnica para cada sub-estágio do método explícito de Runge-Kutta de quarta ordem empregado no estudo de esquemas de diferenças finitas usando a formulação vorticidade.

Nos anos 60, Chorin [6] observou que para escoamentos incompressíveis, a pressão não tem um papel termodinâmico. Ela tem por função forçar a condição de incompressibilidade para o fluido. Esta observação motivou um esquema de discretização baseado na separação de operadores e é conhecido como o método de projeção ou o método de passo fracionário. Neste método, os cálculos da velocidade e da pressão são realizados por intermédio de dois passos: um preditor e outro corretor. No primeiro passo, um campo de velocidade auxiliar é calculado, resolvendo-se a equação da quantidade de movimento sem considerar o termo da pressão e a condição de incompressibilidade. No segundo passo, de acordo com o teorema de decomposição de Hodge (Chorin e Marsden [7]), a velocidade auxiliar é projetada no espaço dos campos vetoriais com divergente zero para determinar a pressão (ou correção de pressão) e a velocidade correta (que satisfaz a condição de incompressibilidade).

\subsection{Métodos de projeção: explícitos e semi-implícitos}

Como foi descrito na Seção anterior, o método de projeção está baseado no desacoplamento do cálculo da velocidade e da pressão. Várias propostas foram apresentadas [3], [6], [23], [46] e as principais diferenças entre elas residem nas expressões para o cálculo da velocidade auxiliar e para o passo de projeção. Exemplos da aplicabilidade dos métodos de projeção em problemas práticos podem ser encontrados em [10], [11], [27], [28], [34], [36], [43]. A seguir, apresentam-se dois métodos explícitos, um de primeira e outro de segunda ordem no tempo, e um semi-implícito de segunda ordem no tempo. 


\subsubsection{Um método explícito de primeira ordem no tempo}

O método explícito a ser apresentado está baseado na aproximação de primeira ordem no tempo, (4.3), para as equações de Navier-Stokes. Estas aproximações serão resolvidas através do método de projeção, que será dividido em três passos:

Passo 1: Cálculo de uma velocidade auxiliar, $\mathbf{u}^{*}$, da equação da quantidade de movimento do sistema (4.3), sem considerar a condição de incompressibilidade:

$$
\begin{aligned}
\rho\left(\frac{\mathbf{u}^{*}-\mathbf{u}^{n}}{d t}+(\mathbf{u} \cdot \nabla \mathbf{u})^{n}\right) & =-\nabla q+\nabla \cdot[2 \mu \mathbf{d}]^{n}+\mathbf{f}^{n}, \\
\mathbf{C C}\left(\mathbf{u}^{*}\right) & =\mathbf{h}_{\mathbf{u}^{*}},
\end{aligned}
$$

onde $q$ é uma aproximação para $p^{n+1}$ e CC é algum tipo de condição de contorno para a velocidade auxiliar.

Passo 2: Projeção da velocidade auxiliar:

$$
\begin{aligned}
\mathbf{u}^{*} & =\mathbf{u}^{n+1}+\frac{\Delta t}{\rho} \nabla \phi, \\
\nabla \cdot \mathbf{u}^{n+1} & =0 .
\end{aligned}
$$

Para calcular $\mathbf{u}^{n+1}$ de (4.7) é necessário conhecer o campo $\phi$. Aplicando-se o operador divergente à equação (4.7) e usando a igualdade (4.8), tem-se uma equação de Poisson para $\phi$

$$
\nabla^{2} \phi=\frac{\rho}{\Delta t} \nabla \cdot \mathbf{u}^{*}
$$

A solução desta equação será obtida uma vez definida a condição de contorno para $\phi$.

Passo 3: Determinação da pressão (atualização da pressão). Para isto será usado o seguinte fato: equações (4.5) e (4.7) aproximam a equação (4.3). Portanto, substituindose (4.7) em (4.5) e igualando-se com a equação (4.3) obtém-se

$$
p^{n+1}=\phi+q \text {. }
$$


Para este caso, o método de projeção requer condições de contorno numéricas para as variáveis não físicas, $\mathbf{u}^{*}$ e $\phi$, e estas devem ser consistentes para determinar aproximações da ordem do método [4].

Atualmente este método é usado, embora tenha uma forte restrição no tamanho de passo. No ICMC/USP é usado um método similar ao descrito para desenvolver um ambiente de simulação de escoamentos de fluidos em duas e três dimensões com superfície livre, denominado FreFlow ([43]).

\subsubsection{Um método explícito de segunda ordem no tempo}

A equação (4.4) é uma aproximação de segunda ordem no tempo das equações de NavierStokes. A avaliação dos termos advectivos e difusivos no tempo $t^{n+1 / 2}$ pode ser feita usando o campo de velocidade no tempo $n+1 / 2$.

Para determinar o campo de velocidade no tempo $n+1 / 2$, pode-se aplicar um método de Euler explícito para as equações de Navier-Stokes, com tamanho de passo igual à metade do tamanho de passo total. Logo, na dedução deste método explícito, além de usar a aproximação no tempo, equação (4.4), é usada a seguinte aproximação

$$
\begin{cases}\rho\left(\frac{\mathbf{u}^{n+1 / 2}-\mathbf{u}^{n}}{\Delta t}+(\mathbf{u} \cdot \nabla \mathbf{u})^{n}\right) & =-\nabla p^{n}+\nabla \cdot[2 \mu \mathbf{d}]^{n}+\mathbf{f}^{n} \\ \nabla \cdot \mathbf{u}^{n+1 / 2} & =0 .\end{cases}
$$

As duas aproximações no tempo, (4.4) e (4.11), correspondem à aplicação de um método explícito de Runge-Kutta de segunda ordem no tempo para as equações de NavierStokes.

Cada sub-estágio do método de Runge-Kutta será resolvida via um método de projeção, similar ao desenvolvido na seção 4.2.1. No primeiro estágio, calculam-se uma velocidade auxiliar para $\mathbf{u}^{n+1 / 2}$ e uma correção de pressão $\phi^{n+1 / 2}$. Com estas, determinam-se a velocidade e a pressão: $\mathbf{u}^{n+1 / 2}$ e $p^{n}$. O passo de atualização para o campo da pressão, $p^{n}$, é similar à equação (4.10), com $\phi=\phi^{n+1 / 2}$. Similarmente, no segundo estágio, calculamse uma velocidade auxiliar para a velocidade $\mathbf{u}^{n+1}$ e uma correção para a pressão $\phi^{n+1}$. Estas permitirão o cálculo de $\mathbf{u}^{n+1}$ e $p^{n+1 / 2}$. Aqui, o passo de atualização para a pressão continua sendo da mesma forma que na anterior, exceto que $\phi=\phi^{n+1}$. As atualizações em cada estágio são consistentes com as equações de Navier-Stokes aproximadas no tempo. Logo, um passo no tempo neste método explícito leva à aplicação de dois métodos de projeção e, portanto, a dois cálculos de velocidades auxiliares e duas soluções de equações 
de Poisson.

O método descrito acima foi usado por Fernandez-Feria e Sanmiguel-Rojas [12] em escoamentos abertos de fluidos incompressívies onde a condição de contorno de Dirichlet para a pressão foi adotada em partes do bordo do domínio.

\subsubsection{Método semi-implícito de segunda ordem no tempo}

Métodos de projeção explícitos têm uma forte restrição no tamanho do passo no tempo. Esta restrição pode ser evitada usando-se métodos de projeção semi-implícitos.

Os métodos de projeção semi-implícitos foram motivados pela seguinte aproximação de segunda ordem no tempo para as equações de Navier-Stokes:

$$
\begin{cases}\rho\left(\frac{\mathbf{u}^{n+1}-\mathbf{u}^{\mathbf{n}}}{d t}+(\mathbf{u} \cdot \nabla \mathbf{u})^{n+1 / 2}\right) & =-\nabla p^{n+1 / 2}+\nabla \cdot\left[\mu\left(\mathbf{d}^{n}+\mathbf{d}^{n+1}\right)\right]+\mathbf{f}^{n+1 / 2}, \\ \nabla \cdot \mathbf{u}^{n+1} & =0,\end{cases}
$$

onde o termo advectivo é avaliado mediante um processo de extrapolação (por exemplo, usando-se Adams Bashforth de segunda ordem)

O sistema (4.12) é uma outra versão do sistema (4.4), onde o termo difusivo é aproximado usando a regra dos trapezios:

$$
\mathrm{d}^{n+1 / 2} \approx \frac{\mathrm{d}^{n}+\mathrm{d}^{n+1}}{2} .
$$

Foi nos anos 80, que muitas propostas apareceram para resolver estas equações discretizadas no tempo. Trabalhos analíticos e numéricos mostraram que os métodos de projeção introduzidos para resolver estas equações tinham uma aproximação de segunda ordem para a velocidade e de primeira ordem para a pressão (algumas referências bibliográficas em [4]).

No ano de 2001, Brown et al. [4] publicaram um trabalho analítico e numérico, referente a três métodos de projeção para as equações semi-implicitas e deduziram que a fonte de aproximação de primeira ordem estava na inconsistente atualização para a pressão. Eles mostraram que a expressão para atualizar a pressão, para os três métodos de projeção semi-implícitos, é dada por:

$$
p^{n+1 / 2}=\phi+q-\frac{\mu \Delta t}{\rho} \nabla^{2} \phi
$$


Para um dos métodos de projeção, a condição de contorno para a velocidade auxiliar também teve que ser modificada para obter-se aproximação de segunda ordem para a pressão.

\subsection{Discretizações adotadas}

O método de solução numérica adotado para resolver as equações de interação no tempo está baseado no método de Runge-Kutta explícito de segunda ordem, enquanto que no espaço é baseado no método de diferenças finitas de segunda ordem. A seguir são apresentados alguns detalhes das discretizações adotadas para o domínio e para os termos das equações do sistema.

\subsubsection{Discretização no espaço}

Um domínio retangular, $\Omega$, é usado para estudar a interação fluido-estrutura. Nesse domíno encontram-se o fluido e a(s) estrutura(s) imersa(s) e, no Modelo Físico Virtual $\Omega$ será o novo domínio para o fluido onde a estrutura é representada por uma curva que define o contorno que esta possui.

A discretização adotada para $\Omega$ está baseada em uma malha regular. Nessa malha, as variáveis são distribuídas na forma apresentada por Harlow e Welch [15], conhecida como a malha MAC. Essa malha é composta por células com arestas em duas dimensões, $\Delta x$ e $\Delta y$ (por simplicidade, $\Delta x=\Delta y$ ). Em uma célula com coordenadas $(i, j$ ), as variáveis que descrevem campos escalares são considerados nos centros das células, enquanto as componentes dos campos vetoriais são discretizadas nos lados das células: a componente no eixo $x$ nas faces laterais distam $( \pm) \frac{\Delta x}{2}$ do centro e a componente no eixo $y$ nas faces superior e inferior distam $( \pm) \frac{\Delta y}{2}$ do centro.

Na Figura 4.1 (esquerda) apresenta-se uma típica célula MAC com os correspondentes índices fracionários e na Figura 4.1 (direita) apresenta-se a mesma célula usando índices inteiros. Esta última notação tem por objetivo evitar índices fracionários e facilitar a implementação computacional.

A interface (curva) onde serão calculada a densidade de força lagrangiana do MFV, é discretizada em um conjunto de pontos igualmente espaçados com espaçamento igual à metade do espaçamento da malha euleriana (Peskin [34]). O curva discretizada e os pontos 
escolhidos são denominados de malha lagrangiana e pontos lagrangianos, respectivamente.
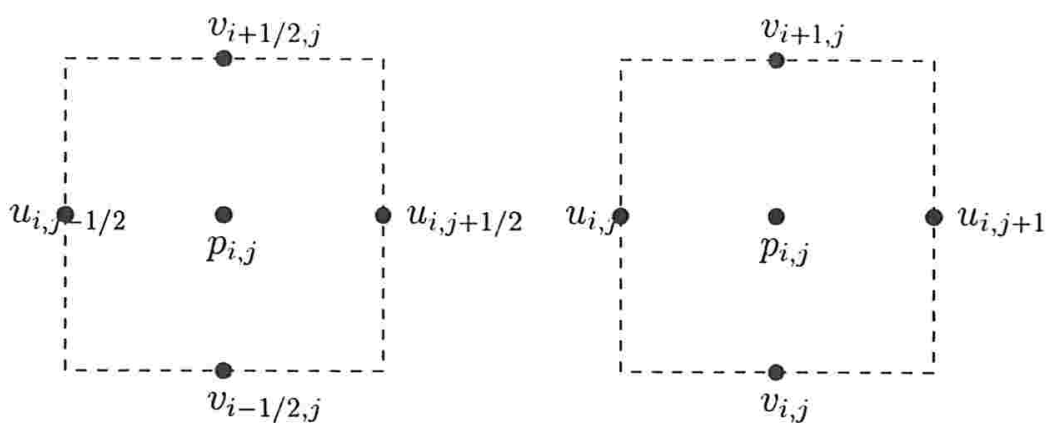

Figura 4.1: Típica célula MAC: índice fracionário (esquerda) e adoção de índices inteiros para o processo de programação computacional (direita).

Para discretizar os termos contendo derivadas no espaço das equações de Navier-Stokes: termos convectivo, difusivo e gradiente de pressão, são empregadas diferenças finitas centradas de segunda ordem. No Apêndice A são mostradas as expressões discretas para esse termos.

\subsubsection{Discretização temporal da densidade de força lagrangiana}

A densidade de força lagrangiana calculada pelo Modelo físico virtual, é dada por:

$$
\mathbf{F}\left(\mathbf{X}_{k}, t\right)=\left(\rho \frac{\partial \mathbf{u}}{\partial t}+\rho(\mathbf{u} \cdot \nabla \mathbf{u})+\nabla p-\nabla \cdot(2 \mu \mathbf{d})\right)\left(\mathbf{X}_{k}, t\right)
$$

Esta pode ser expressa da seguinte forma

$$
\mathbf{F}\left(\mathbf{X}_{k}, t\right)=\rho \frac{\partial \mathbf{u}\left(\mathbf{X}_{k}, t\right)}{\partial t}+\mathbf{G}\left(\mathbf{X}_{k}, t\right)
$$

onde:

$$
\mathbf{G}=\rho(\mathbf{u} \cdot \nabla \mathbf{u})+\nabla p-\nabla \cdot(2 \mu \mathbf{d}) .
$$

Lima e Silva et al.[25] chamaram o primeiro termo do lado direito da equação (4.14) de força de aceleração e discretizaram-lo da seguinte forma:

$$
\mathbf{F}_{a}\left(\mathrm{X}_{k}, t\right)=\rho \frac{\partial \mathrm{u}\left(\mathrm{X}_{k}, t\right)}{\partial t}=\rho\left(\frac{\mathrm{V}_{k}-\mathrm{u}_{f k}}{\Delta t}\right),
$$


onde: $\mathbf{V}_{k}$ e $\mathbf{u}_{f k}$ são a velocidade da interface e a velocidade do fluido no ponto $\mathrm{X}_{k}$, respectivamente. Esta expressão é válida se a velocidade $\mathrm{V}_{k}$ da interface é conhecida no respectivo tempo de integração.

A aplicação do método de Runge-Kutta de segunda ordem implica na determinação de duas vezes a densidade de força lagrangiana, uma em cada estágio do método. A expressão para a força de aceleração da equação (4.15) é aproximada e esta depende do estágio no qual está-se calculando e da condição de movimento da estrutura (velocidade prescrita ou não). A seguir, são dadas expressões para a densidade de força lagrangiana baseada no estágio e na condição do movimento para a estrutura.

\section{Caso 1: velocidade não prescrita}

Em muitos problemas de interação fluido-estrutura a velocidade da interface ou da estrutura não é conhecida até as equações dos sistema serem resolvidas.

O cálculo da densidade de força lagrangiana faz uso da velocidade da interface para impor a condição de contorno na interface. Devido ao fato desta não ser conhecida no instante atual, o cálculo da densidade de força lagrangiana deve ser determinado mediante processos que dependem do método numérico empregado para o sistema.

Aqui usa-se o método de Runge-Kutta explícito de segunda ordem para resolver as equações do sistema, portanto, informações do tempo anterior são usadas para determinar a densidade de força lagrangiana.

O cálculo da densidade de força lagrangiana no primeiro estágio emprega os pontos $\left(\mathbf{X}_{k}^{n}, t^{n}\right)$. Para aproximar a força de aceleração usa-se uma aproximação regressiva. Logo, a densidade de força lagrangiana tem a seguinte forma:

$$
\mathbf{F}\left(\mathbf{X}_{k}^{n}, t^{n}\right)=\rho\left(\frac{\mathbf{u}^{n}\left(\mathbf{X}_{k}^{n}\right)-\mathbf{u}^{n-1 / 2}\left(\mathbf{X}_{k}^{n}\right)}{0.5 \Delta t}\right)+\mathbf{G}\left(\mathbf{X}_{k}^{n}, t^{n}\right) .
$$

Na equação (4.17), os campos de velocidade $\mathbf{u}^{n}\left(X_{k}^{n}\right)$ e $\mathbf{u}^{n-1 / 2}\left(\mathbf{X}_{k}^{n}\right)$ devem ser definidos com a condição de se impor a condição de contorno na interface. As seguintes relações são usadas para estes campos:

$$
\begin{aligned}
\mathrm{u}^{n}\left(\mathrm{X}_{k}^{n}\right) & =\mathrm{V}_{k}^{n}, \\
\mathrm{u}^{n-1 / 2}\left(\mathrm{X}_{k}^{n}\right) & \approx \mathrm{u}_{f k}^{n}\left(\mathrm{X}_{k}^{n}\right) .
\end{aligned}
$$

A expressão para a densidade de força no primeiro estágio é dada por:

$$
\mathbf{F}\left(\mathbf{X}_{k}^{n}, t^{n}\right)=\rho\left(\frac{\mathbf{V}_{k}^{n}-\mathbf{u}_{f k}^{n}}{0.5 \Delta t}\right)+\mathbf{G}\left(\mathbf{X}_{k}^{n}, t^{n}\right)
$$


Para o segundo estágio a densidade de força lagrangiana é aplicada nos pontos $\left(\mathrm{X}_{k}^{n+1 / 2}, t^{n+1 / 2}\right)$. Neste caso, a força de aceleração é aproximada usando diferença centrada. Então

$$
\mathbf{F}\left(\mathbf{X}_{k}^{n+1 / 2}, t^{n+1 / 2}\right)=\rho\left(\frac{\mathbf{u}^{n+1}-\mathbf{u}^{n}}{\Delta t}\right)\left(\mathbf{X}_{k}^{n+1 / 2}\right)+\mathbf{G}\left(\mathbf{X}_{k}^{n+1 / 2}, t^{n+1 / 2}\right) .
$$

Similarmente, as velocidades $\mathrm{u}^{n+1}\left(\mathrm{X}_{k}^{n+1 / 2}\right)$ e $\mathbf{u}^{n}\left(\mathrm{X}_{k}^{n+1 / 2}\right)$ devem ser calculadas com a condição de satisfazer a condição de contorno na interface. Para isto, usam-se as seguintes relações:

$$
\begin{aligned}
\mathrm{u}^{n+1}\left(\mathrm{X}_{k}^{n+1 / 2}\right) & \approx 2 \mathbf{u}^{n+1 / 2}\left(\mathrm{X}_{k}^{n+1 / 2}\right)-\mathbf{u}^{n}\left(\mathrm{X}_{k}^{n+1 / 2}\right) \\
\mathrm{u}^{n}\left(\mathrm{X}_{k}^{n+1 / 2}\right) & \approx \mathbf{u}_{f k}^{n+1 / 2}\left(\mathrm{X}_{k}^{n+1 / 2}\right) \\
\mathrm{u}^{n+1 / 2}\left(\mathrm{X}_{k}^{n+1 / 2}\right) & =\mathrm{V}_{k}^{n+1 / 2}
\end{aligned}
$$

Substituindo-se tais expressões na equação (4.19) e fazendo operações obtém-se:

$$
\mathbf{F}\left(\mathbf{X}_{k}^{n+1 / 2}, t^{n+1 / 2}\right)=\rho\left(\frac{\left.\mathbf{V}_{k}^{n+1 / 2}-\mathbf{u}_{f k}^{n+1 / 2}\right)}{0.5 \Delta t}\right)+\mathbf{G}\left(\mathbf{X}_{k}^{n+1 / 2}, t^{n+1 / 2}\right) .
$$

\section{Caso 2: velocidade prescrita}

Exemplos de estruturas com velocidade conhecida, isto é, velocidade prescrita, podem ser encontrados em muitos sistemas de interação. Por exemplo, rotação de cilindros, helices de um helicóptero, etc.

Para estas estruturas com velocidade prescrita, a determinação de sua posição no tempo pode ser feita integrando-se as equações de translação e de rotação. Por exemplo, no caso de o movimento da estrutura ser unicamente de translação, a posição da estrutura é obtida, resolvendo-se:

$$
\frac{d \mathrm{X}_{k}}{d t}=\mathrm{V}_{k}
$$

onde $\mathrm{V}_{k}$ é a velocidade dada da interface.

Tanto a velocidade como a posição da estrutura são conhecidas em todo tempo e estas podem ser usadas na determinação da densidade de força lagrangiana. Assim, no primeiro estágio do método, a densidade de força lagrangiana é aplicada nos pontos $\left(\mathrm{X}_{k}^{n+1 / 2}, t^{n}\right)$. Para a força de aceleração, é usada uma aproximação progressiva. Logo, a densidade de força lagrangiana terá a seguinte expressão:

$$
\mathbf{F}\left(\mathbf{X}_{k}^{n+1 / 2}, t^{n}\right)=\rho\left(\frac{\mathbf{u}^{n+1 / 2}-\mathbf{u}^{n}}{0.5 \Delta t}\right)\left(\mathbf{X}_{k}^{n+1 / 2}\right)+\mathbf{G}\left(\mathbf{X}_{k}^{n+1 / 2}, t^{n}\right)
$$


Novamente, campos vetoriais devem ser aproximados em (4.21). Usando-se as seguintes relações

$$
\begin{aligned}
\mathrm{u}^{n+1 / 2}\left(\mathrm{X}_{k}^{n+1 / 2}\right) & =\mathrm{V}_{k}^{n+1 / 2}, \\
\mathrm{u}^{n}\left(\mathrm{X}_{k}^{n+1 / 2}\right) & =\mathrm{u}_{f k}^{n},
\end{aligned}
$$

tem-se

$$
\mathbf{F}\left(\mathbf{X}_{k}^{n+1 / 2}, t^{n}\right)=\rho\left(\frac{\mathbf{V}_{k}^{n+1 / 2}-\mathbf{u}_{f k}^{n}}{0.5 \Delta t}\right)+\mathbf{G}\left(\mathbf{X}_{k}^{n+1 / 2}, t^{n}\right) .
$$

No segundo estágio, a densidade de força lagrangiana é aplicada nos pontos $\left(\mathbf{X}_{k}^{n+1}, t^{n+1 / 2}\right)$ e a derivada temporal é aproximada por diferenças centradas

$$
\mathbf{F}\left(\mathbf{X}_{k}^{n+1}, t^{n+1 / 2}\right)=\rho\left(\frac{\mathbf{u}^{n+1}-\mathbf{u}^{n}}{\Delta t}\right)\left(\mathbf{X}_{k}^{n+1}\right)+\mathbf{G}\left(\mathbf{X}_{k}^{n+1}, t^{n+1 / 2}\right),
$$

onde

$$
\begin{aligned}
\mathbf{u}^{n+1}\left(\mathrm{X}_{k}^{n+1}\right) & =\mathbf{V}_{k}^{n+1}, \\
\mathbf{u}^{n}\left(\mathrm{X}_{k}^{n+1}\right) & \approx \mathbf{u}_{f k}^{n+1 / 2} .
\end{aligned}
$$

Logo, a densidade de força lagrangiana neste segundo estágio é dada por:

$$
\mathbf{F}\left(\mathbf{X}_{k}^{n+1}, t^{n+1 / 2}\right)=\rho\left(\frac{\mathbf{V}_{k}^{n+1}-\mathbf{u}_{f k}^{n+1 / 2}}{\Delta t}\right)+\mathbf{G}\left(\mathbf{X}_{k}^{n+1}, t^{n+1 / 2}\right) .
$$

\subsubsection{Condição de estabilidade}

A estabilidade do método depende do passo no tempo. Para métodos explícitos de RungeKutta aplicados às equações de Navier-Stokes, a restrição do passo no tempo para o método ser estável é dada por ([31]):

$$
\Delta t=\tau \min \left(\Delta t_{1}, \Delta t_{2}\right)
$$

onde

$$
\begin{aligned}
& \Delta t_{1}<\min \left(\frac{\Delta x}{|u|_{\max }}, \frac{\Delta y}{|v|_{\max }}\right), \\
& \Delta t_{2}<\frac{1}{2 \nu} \frac{(\Delta x)^{2}(\Delta y)^{2}}{(\Delta x)^{2}+(\Delta y)^{2}},
\end{aligned}
$$

e $\tau$ é um fator de segurança, cujo valor está no intervalor $[0,2: 0,6] ;|u|_{\max }$ e $|v|_{\max }$ são o valor da norma do máximo das componentes $u$ e $v$ da velocidade, respectivamente. A restrição (4.26) é devida ao termo advectivo, enquanto que a restrição (4.27) é devida ao termo difusivo das equações de Navier-Stokes. 


\subsection{Condições de contorno numéricas}

As equações de Navier-Stokes requerem, além de uma condição inicial, condições de contorno para a velocidade, para serem completamente determinadas. Estas podem ser, entre outras, condições de contorno do tipo Dirichlet e/ou Neumann.

Devido ao uso do método de projeção para resolver as equações de Navier-Stokes, condições de contorno numéricas adicionais são requeridas para a solução numérica ser determinada. Estas condições de contorno adicionais tem origem no desacoplamento do cálculo da velocidade e da pressão das equações de Navier-Stokes, que empregam duas variáveis adicionais, $\mathbf{u}^{*}$ e $\phi$. Estas condições de contorno são numéricas, devido à natureza destas variáveis auxiliares, não representando propriedades físicas do escoamento.

Alguns autores que usaram o método de projeção para resolver as equações do escoamento adotaram condições de contorno periódicas para o campo de velocidade e de pressão do fluido $[34,38,37]$. Neste caso, é obvia a escolha da condição de contorno periódica para as variáveis $\phi$ e $\mathbf{u}^{*}$. Para outras condições de contorno das equações do fluido deve-se determinar condições de contorno apropriadas para estas variáveis. Devido à relação (4.7), condições de contorno para $\phi$ e $\mathbf{u}^{*}$ devem ser consistentes com a condição de contorno para a velocidade do fluido.

No caso de se ter condições de contorno do tipo Dirichlet para a velocidade, isto é,

$$
\mathbf{u}=\mathbf{u}_{\mathbf{b}}, \quad \mathbf{x} \in \partial \Omega
$$

e supondo que a condição de contorno para a velocidade auxiliar, $\mathbf{u}^{*}$, é dada pela mesma expressão que para a velocidade do fluido, isto é,

$$
\mathbf{u}^{*}=\mathbf{u}_{\mathbf{b}}, \quad \mathrm{x} \in \partial \Omega,
$$

pode-se determinar uma condição de contorno para a variável $\phi$ usando-se a equação (4.7).

Aplicando-se o produto interno na equação (4.7) com o vetor normal obtém-se a seguinte condição de contorno para a variável $\phi$,

$$
\nabla \phi \cdot \mathbf{n}=0
$$

Esta é uma condição de contorno do tipo Neumann homogênea para a variável $\phi$.

Condições de contorno não consistentes para as variáveis auxiliares e/ou atualizaçōes não corretas para a pressão podem influenciar na convergência do método de projeção 
adotado. Brown et al. [4] apresentaram modificações nas condições de contorno e/ou atualizações da pressão que eram usadas por três métodos de projeção semi-implícitos de segunda ordem no tempo e no espaço e que tinham uma convergência de segunda ordem para a velocidade e de primeira ordem para a pressão. Com estas modificações, foram obtidas convergência de segunda ordem para a pressão nos três métodos de projeção.

\subsection{Algoritmo de Runge-Kutta para a interação fluido- estrutura}

O algoritmo para o estágio $l=1,2$ do RKE2 aplicado é:

\section{Dados: $\mathbf{X}_{k}, \mathbf{V}_{c}, w, \mathbf{X}_{c}, \theta$ e $\mathbf{u}, q \approx p$ em $t^{n+\frac{l-1}{2}}$}

1. Calculam-se $\mathrm{F}^{n+\frac{l-1}{2}}$ e $\mathbf{f}^{n+\frac{l-1}{2}}$ usando equação (3.4) e (3.16), respectivamente.

2. Calcula-se $\left(\mathbf{u}^{*}\right)^{n+\frac{1}{2}}$ :

$$
\rho\left(\frac{\left(\mathbf{u}^{*}\right)^{n+\frac{l}{2}}-\mathbf{u}^{n}}{\alpha_{l} \Delta t}+(\mathbf{u} \cdot \nabla \mathbf{u})^{n+\frac{l-1}{2}}\right)=-\nabla q^{n+\frac{l-1}{2}}+\nabla \cdot[2 \mu \mathrm{d}]^{n+\frac{l-1}{2}}+\mathbf{f}^{n+\frac{l-1}{2}} .
$$

3. Determinam-se $\mathbf{u}^{n+\frac{l}{2}}$ e $p^{n+\frac{l-1}{2}}$ :

$$
\begin{aligned}
\left(\mathbf{u}^{*}\right)^{n+\frac{l}{2}} & =\mathbf{u}^{n+\frac{l}{2}}+\frac{\alpha_{l} \Delta t}{\rho} \nabla \phi_{l}, \\
\nabla \cdot \mathbf{u}^{n+\frac{l}{2}} & =0, \\
p^{n+\frac{l-1}{2}} & =q^{n+\frac{l-1}{2}}+\phi_{l} .
\end{aligned}
$$

4. Calcula-se $\mathbf{F}_{f c}^{n+\frac{l-1}{2}}$ e $\mathbf{T}_{f c}^{n+\frac{l-1}{2}}$ usando-se equação (3.21) e (3.22), respectivamente.

5. Determine-se $\mathbf{V}_{c}^{n+\frac{l}{2}}$ e $w^{n+\frac{1}{2}}$ :

$$
\begin{aligned}
M_{c}\left(\frac{\mathrm{V}_{c}^{n+\frac{l}{2}}-\mathrm{V}_{c}^{n}}{\alpha_{l} \Delta t}\right) & =\mathbf{G}_{c}+\mathbf{F}_{f c}^{n+\frac{l-1}{2}}, \\
I_{c}\left(\frac{w^{n+\frac{l}{2}}-w^{n}}{\alpha_{l} \Delta t}\right) & =\mathrm{T}_{f c}^{n+\frac{l-1}{2}} .
\end{aligned}
$$


6. Determina-se $\mathbf{X}_{c}^{n+\frac{l}{2}}$ e $\theta^{n+\frac{1}{2}}$ :

$$
\begin{aligned}
\frac{\mathrm{X}_{c}^{n+\frac{l}{2}}-\mathrm{X}_{c}^{n}}{\alpha_{l} \Delta t} & =\mathrm{V}_{c}^{n+\frac{l-1}{2}} \\
\frac{\theta^{n+\frac{l}{2}}-\theta^{n}}{\alpha_{l} \Delta t} & =w^{n+\frac{l-1}{2}}
\end{aligned}
$$

7. Determina-se $\mathbf{X}_{k}^{n+\frac{l}{2}}$

$$
\mathrm{X}_{k}^{n+\frac{l}{2}}=\mathrm{X}_{c}^{n+\frac{l}{2}}+\mathbf{A}\left(\mathrm{X}_{k}^{n+\frac{l-1}{2}}-\mathrm{X}_{c}^{n+\frac{l-1}{2}}\right)
$$

onde A é uma matriz de rotação que depende do ângulo $d \theta=\theta^{n+\frac{l}{2}}-\theta^{n}$.

$$
\alpha_{1}=0.5 \text { e } \alpha_{2}=1.0
$$

As equações (4.29) e (4.30) do passo 3 do algoritmo geram uma equação de Poisson para $\phi_{l}$. Esta equação é resolvida usando-se rotinas do FISHPACK [40]. 


\section{Resultados numéricos}

Neste capítulo apresentam-se resultados de validações numéricas e de estudos do comportamento da modelagem matemática para interação fluido-estrutura rígida via o Método da Fronteira Imersa/Modelo Físico Virtual (MFI/MFV).

Este capítulo está organizado da seguinte forma: na primeira seção são apresentados os elementos matemáticos usados para avaliar o comportamento quantitativo da metodologia. A validação do método de solução das equações do fluido é apresentada na Seção 5.2 . Na Seção 5.3 á apresentada a validação do MFV com um problema de interação parcial. Os resultados de um estudo do comportamento da aplicação do MFV em um problema de interação total para o qual não são conhecidas soluções analíticas e/ou experimentais são apresentados na Seção 5.4. Finalmente, são apresentados os resultados da aplicação do MFV para um problema de interação parcial e total na Seção 5.5

\subsection{Preliminares: normas e estudo de convergência}

Neste trabalho são apresentadas simulações bidimensionais de problemas de interação fluido-estrutura. O domínio do estudo é um conjunto retangular $\Omega=[a, b] \times[c, d] \subset R^{2}$. Uma malha regular para o domínio é usada e as variáveis do fluido são discretizadas numa malha MAC (Mark and Cell). Nesta malha MAC as variáveis que descrevem campos escalares são considerados no centro das células, enquanto que as componentes dos campos vetoriais são considerados nos faces das células.

Em uma malha MAC três conjuntos de pontos podem ser diferenciados: o conjunto de pontos onde variáveis escalares do fluido são calculados, $\Omega_{p}^{h}$; o conjunto de pontos onde a primeira componente das variáveis vetoriais do fluido são calculadas, $\Omega_{u}^{h}$ e o conjunto de 
pontos onde a segunda componente das variáveis vetoriais são calculados, $\Omega_{v}^{h}$. Normas \|\|$_{1}$, \|\|$_{2}$ e \|\|$_{\infty}$ podem ser definidas para as variáveis escalares e vetoriais usando os respectivos conjuntos de pontos descritos acima.

As normas \|\|$_{1},\|\|_{2}$ e \|\|$_{\infty}$ para uma variável $\phi$ definida em $\Omega_{p}^{h}$, será denotada por:

$$
\begin{aligned}
\|\phi\|_{q} & =\left(\sum_{\mathbf{k} \in \Omega_{p}^{h}}\left|\phi_{\mathbf{k}}\right|^{q} h_{\mathbf{k}}^{2}\right)^{1 / q}, \quad q=1,2, \\
\|\phi\|_{\infty} & =\max _{\mathbf{k} \in \Omega_{p}^{h}}\left|\phi_{\mathbf{k}}\right|
\end{aligned}
$$

onde $\phi_{\mathbf{k}}$ é o valor de $\phi$ no centro da célula $\mathbf{k}=\left(k_{1}, k_{2}\right)$, de quem o comprimento e largura é $h_{\mathrm{k}}$.

Se $\psi=\left(\psi_{1}, \psi_{2}\right)$ é um vetor variável com $\psi_{1}$ definido em $\Omega_{u}^{h}$ e $\psi_{2}$ definido em $\Omega_{v}^{h}$, então:

$$
\begin{aligned}
\|\psi\|_{q} & =\left(\sum_{\mathbf{i} \in \Omega_{u}^{h}}\left|\psi_{1 \mathbf{i}}\right|^{q} h_{\mathbf{i}}^{2}+\sum_{\mathbf{j} \in \Omega_{v}^{h}}\left|\psi_{2 \mathbf{j}}\right|^{q} h_{\mathbf{j}}^{2}\right)^{1 / q}, \quad q=1,2, \\
\|\psi\|_{\infty} & =\max \left\{\max _{\mathbf{i} \in \Omega_{u}^{h}}\left|\psi_{1 \mathbf{i}}\right|, \max _{\mathbf{j} \in \Omega_{v}^{h}}\left|\psi_{2 \mathbf{j}}\right|\right\} .
\end{aligned}
$$

Para analisar numericamente a convergência do método, será suposto que a solução fornecida por ele tem expansão assintótica nas potências do espaçamento da malha. Nas malhas uniformes empregadas, por exemplo, a função escalar $\phi$ tem solução numérica $\phi(\mathrm{x}, t, h)$ a qual, por hipótese, pode ser escrita na forma assintótica

$$
\phi(\mathbf{x}, t, h)=\phi_{e}(\mathbf{x}, t)+\mathbf{E}_{q}(\mathbf{x}, t) h^{q}+\mathbf{E}_{q+1}(\mathbf{x}, t) h^{q+1}+\ldots
$$

para $h$ suficientemente pequeno, onde $\phi_{e}(\mathbf{x}, t)$ é a solução exata e os coeficientes $\mathbf{E}_{i}(\mathbf{x}, t)$, $i=q, q+1, \ldots$, independem de $h$.

Se a solução exata é conhecida, após ter sido encontrada a solução aproximada $\phi(x, t, h)$ para algum $h$, calcula-se para o mesmo ponto $(\mathbf{x}, t)$ a aproximação $\phi(\mathbf{x}, t, h / 2)$. Assim obtém-se, em primeira aproximação,

$$
\begin{gathered}
\phi(\mathrm{x}, t, h) \approx \phi_{e}(\mathrm{x}, t)+\mathrm{E}_{q}(\mathrm{x}, t) h^{q} \\
\phi(\mathrm{x}, t, h / 2) \approx \phi_{e}(\mathrm{x}, t)+\mathrm{E}_{q}(\mathrm{x}, t) \frac{h^{q}}{2^{q}}
\end{gathered}
$$


Das duas aproximações anteriores, os erros entre a solução exata e as aproximadas para malhas de espaçamentos $h$ e $h / 2$, satisfazem a razão

$$
\frac{\left\|\phi(\mathbf{x}, t, h)-\phi_{e}(\mathbf{x}, t)\right\|}{\left\|\phi(\mathbf{x}, t, h / 2)-\phi_{e}(\mathbf{x}, t)\right\|} \approx 2^{q}
$$

onde a norma utilizada pode ser (5.1) ou (5.2).

Seguindo este raciocínio, para aproximações de funções vetoriais que tem uma expansão assintótica, obtém-se

$$
\frac{\left\|\mathrm{v}(\mathrm{x}, t, h)-\mathrm{v}_{e}(\mathrm{x}, t)\right\|}{\left\|\mathrm{v}(\mathrm{x}, t, h / 2)-\mathrm{v}_{e}(\mathrm{x}, t)\right\|} \approx 2^{q}
$$

onde $\mathbf{v}_{e}(\mathrm{x}, t)$ é a solução exata e, mais uma vez, $\|\cdot\|$ representa qualquer uma das normas, $\|.\|_{2}$ ou $\|.\|_{\infty}$, definidas em (5.3) e (5.4), respectivamente.

Fazendo-se uso de (5.5) (ou (5.6)), tem-se que a razão entre os erros, na malha de espaçamento $h$ e $h / 2$, para métodos de ordem $q=1,2$ e 3 , devem ser aproximadamente 2,4 e 8 , respectivamente.

\subsection{Validação do método de projeção explícito}

Para examinar a resolução numérica das equações de Navier-Stokes separadamente, um problema modelo "suave" é utilizado, evitando-se termos forçantes singulares como o dado em (3.3). Nenhuma fronteira imersa será definida e o termo forçante $\mathbf{f}$ provirá de forças exteriores.

O problema suave a ser usado na verificação numérica da ordem de convergência é definido no domínio $\Omega=[0,1] \times[0,1]$ cuja solução exata para o campo de velocidade e pressão são (Lai [28]):

$$
\begin{aligned}
& \mathbf{u}_{e}(\mathbf{x}, t)=\mathbf{a}_{1} \cdot \sin \left(\mathbf{b}_{1} \cdot \mathbf{x}+w_{1} \cdot t\right)+\mathbf{a}_{2} \cdot \sin \left(\mathbf{b}_{2} \cdot \mathbf{x}+w_{2} \cdot t\right), \\
& p_{e}(\mathbf{x}, t)=c_{1} \cdot \sin \left(\mathbf{b}_{1} \cdot \mathbf{x}+w_{1} \cdot t\right)+c_{2} \cdot \sin \left(\mathbf{b}_{2} \cdot \mathbf{x}+w_{2} \cdot t\right),
\end{aligned}
$$

onde

$$
\begin{aligned}
& \mathbf{a}_{1}=(-1,2), \quad \mathbf{a}_{2}=(2,-2), \\
& \mathbf{b}_{1}=2 \pi(2,1), \quad \mathbf{b}_{2}=2 \pi(1,1), \\
& c_{1}=c_{2}=1, \quad w_{1}=w_{2}=w=0.1 .
\end{aligned}
$$


Com termo forçante dado $\mathbf{f}=\left(f_{x}, f_{y}\right)$, sendo

$$
\begin{aligned}
f_{x}(\mathrm{x}, t)= & \rho[-w(\cos A-2 \cos B)+4 \pi(\sin (A-B)]+2 \pi(2 \cos A+\cos B) \\
& -4 \mu \pi^{2}(5 \sin A-4 \sin B) \\
f_{y}(\mathrm{x}, t)= & \rho[2 \pi(\cos A-\cos B)+4 \pi(2 \sin B \cos A-\sin A \cos B)]+2 \pi(\cos A+\cos B) \\
& -4 \mu \pi^{2}(4 \sin B-10 \sin A),
\end{aligned}
$$

onde

$$
A=4 \pi x+2 \pi y+w t \quad \text { e } \quad B=2 \pi x+2 \pi y+w t
$$

\subsubsection{Caso 1: Condição de Dirichlet para a velocidade}

Neste caso usou-se os seguintes parâmetros para as variáveis do fluido, assim como as condições de contorno numéricas para as variáveis auxiliares do método de projeção correspondentes à condição de contorno para u ( Seção B.2 do Apêndice B)

- $\rho=1,0 \mathrm{~g} / \mathrm{cm}^{3}, \mu=1,0 E-3 \mathrm{~g} / \mathrm{cm} \mathrm{s}$;

- $\mathrm{u}(\mathrm{x}, 0)=\mathrm{u}_{e}(\mathrm{x}, 0)$

- $\Delta t=\Delta x^{2} / 2$

- $\mathrm{CC}\left(\mathrm{u}^{*}\right)=\mathrm{CC}(\mathrm{u})$ e $\frac{\partial \phi}{\partial \mathbf{n}}=0$

- $t_{f}=0,1 \mathrm{~s}$

Na Tabela 5.1 são apresentadas as normas dos erros e seus quocientes consecutivos para o cálculo da velocidade do fluido, com um tempo $t_{f}=0,1$, para malhas de tamanho $N \times N, N=16,32,64,128,256$. Comparando as razões calculadas desta tabela com a restrição (5.6) pode se dizer que o método é uma aproximação de segunda ordem para as considerações adotadas.

\subsubsection{Caso 2: Condição Neumann para a velocidade}

Neste estudo usam-se os mesmos valores para os parâmetros do fluido que no estudo anterior, enquanto que as condições de contorno numéricas para as variáveis auxiliares são diferentes e estas são as seguintes (Seção B.2 do Apêndice B) 
- $\frac{\partial \mathbf{u}^{*}}{\partial \mathbf{n}}=\left.\frac{\partial \mathbf{u}^{*}}{\partial \mathbf{n}} e \phi\right|_{\Gamma}=0$

Usando-se as condições indicadas acima encontrou-se as normas dos erros no cálculo da velocidade mostradas na Tabela 5.2 para um tempo $t_{f}=0.1$ e para as normas $L_{2}$ e $L_{\infty}$ e malhas de tamanho $N \times N, N=16,32,64,128,256$. Nessa mesma Tabela, apresentamse os quocientes das normas indicadas entre malhas de tamanhos $N \times N$ e $2 N \times 2 N$. Observe-se que o valor deste quociente comporta-se como dado pela equação (5.6).

\begin{tabular}{|l|l|l|l|l|}
\hline Malha & $\left\|\mathbf{u}-\mathbf{u}_{a}\right\|_{L_{2}}$ & razão & $\left\|\mathbf{u}-\mathbf{u}_{a}\right\|_{L_{\infty}}$ & razão \\
\hline 16 & $3.631986 \mathrm{e}-01$ & & $8.469929 \mathrm{e}-01$ & \\
32 & $1.006141 \mathrm{e}-01$ & 3.6098181 & $3.312879 \mathrm{e}-01$ & 2.5566672 \\
64 & $2.711418 \mathrm{e}-02$ & 3.7107558 & $1.746493 \mathrm{e}-01$ & 1.8968751 \\
128 & $6.149209 \mathrm{e}-03$ & 4.4093769 & $3.503684 \mathrm{e}-02$ & 4.9847332 \\
256 & $1.456337 \mathrm{e}-03$ & 4.2223805 & $6.034676 \mathrm{e}-03$ & 5.805919 \\
\hline
\end{tabular}

Tabela 5.1: Método de projeção com condições de contorno do tipo Dirichlet para a velocidade: erros cometidos no campo de velocidade e razão entre eles, no tempo $t_{f}=0,1 \mathrm{~s}$.

\begin{tabular}{|l|l|l|l|l|}
\hline Malha & $\left\|\mathbf{u}-\mathbf{u}_{a}\right\|_{L_{2}}$ & razão & $\left\|\mathbf{u}-\mathbf{u}_{a}\right\|_{L_{\infty}}$ & razão \\
\hline 16 & $7.820090 \mathrm{e}-01$ & & $2.498489 \mathrm{e}+00$ & \\
32 & $1.952529 \mathrm{e}-01$ & 4,0051082 & $4.422158 \mathrm{e}-01$ & 5,6499315 \\
64 & $4.087282 \mathrm{e}-02$ & 4,7770841 & $9.754564 \mathrm{e}-02$ & 4,5334246 \\
128 & $1.416421 \mathrm{e}-02$ & 2,8856406 & $3.212181 \mathrm{e}-02$ & 3,0367417 \\
256 & $2.790854 \mathrm{e}-03$ & 5,0752243 & $6.581764 \mathrm{e}-03$ & 4,8804257 \\
\hline
\end{tabular}

Tabela 5.2: Método de projeção e condições de contorno do tipo Neumann para a velocidade: erros cometidos, no campo de velocidade, e razão entre eles no tempo $t_{f}=0,1 \mathrm{~s}$.

Para ambas as condições de contorno da velocidade obteve-se que o método é de segunda ordem, a ordem esperada teoricamente.

\subsection{Validação do MFI/MFV: Escoamento de Couette Circular}

Problemas físicos com solução analítica e/ou experimental são usados para validar modelagens matemáticas. Por exemplo, Arruda [1] validou o modelagem matemática do MFV 
usando dois problemas conhecidos: escoamento de Poiseuille, cuja solução analítica é conhecida e escoamento em uma cavidade, cujos resultados experimentais são reportados. Os resultados apresentados por Arruda tiveram boa concordância com a solução analítica e solução experimental.

Um outro problema cuja solução analítica é conhecida é o escoamento de Couette circular. Este problema é um caso especial do escoamento de Couette Taylor, que consiste no escoamento de um fluido na região entre de dois cilindros concêntricos. Um ou ambos cilindros giram com velocidades constantes ao longo do eixo comum (Figura 5.1-(a)).

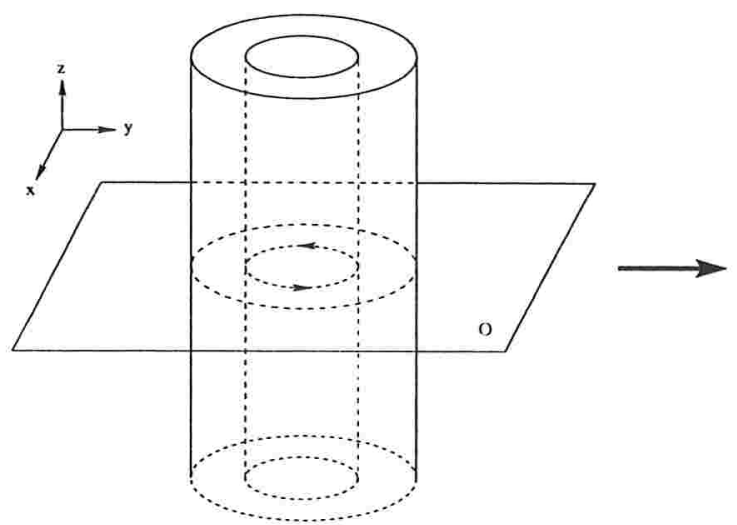

(a)

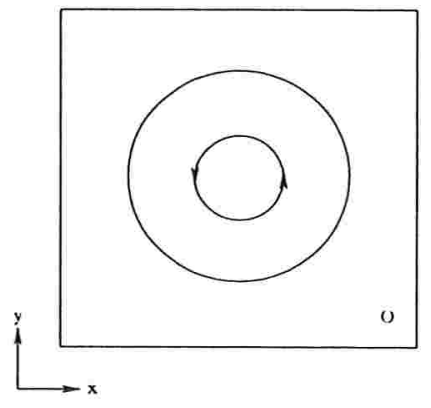

(b)

Figura 5.1: Plano transversal " $\Omega$ " ao escoamento de Couette circular (a); domínio bidimensional, $\Omega$, considerado na modelagem do MFV (b).

O escoamento de Couette circular corresponde ao caso em que o regime do escoamento é estacionário. Este escoamento acontece se o número de Taylor $T_{a}<100$. No caso do cilindro interno estar em movimento e o externo parado, o número de Taylor tem a seguinte definição:

$$
T_{a}=\frac{\rho\left(w_{1} R_{1}\right) g a p}{\mu}
$$

onde: gap é a distância de separação entre os cilindros; $w_{1}$ e $R_{1}$ são a velocidade angular e o raio do cilindro interno.

Em geral, a solução exata para o escoamento de Couette circular é dada em coordenadas cilíndricas por:

$$
\mathbf{u}=\left(u_{r}, u_{\theta}, u_{z}\right)=(0, v(r), 0)
$$


onde:

$$
v(r)=A r+\frac{B}{r}
$$

sendo $A$ e $B$ constantes a serem determinados pela condição de contorno nas paredes. $\mathrm{O}$ valor dessas constantes são:

$$
\begin{aligned}
A & =\frac{w_{2} r_{2}^{2}-w_{1} r_{1}^{2}}{r_{2}^{2}-r_{1}^{2}}, \\
B & =\frac{\left(w_{1}-w_{2}\right) r_{1}^{2} r_{2}^{2}}{r_{2}^{2}-r_{1}^{2}} .
\end{aligned}
$$

Uma expressão para a velocidade em coordenadas cartesianas é dada por:

$$
\mathbf{u}=v(r)(-\operatorname{sen}(\theta), \cos (\theta))
$$

\section{Parâmetros de simulação}

Para representar a presença dos cilindros do escoamento de Couette circular no domínio bidimensional euleriano são usadas duas malhas lagrangianas. A primeira malha representa o primeiro cilindro interno, de raio $R_{1}$ e a velocidade de rotação $w_{1}$ a segunda malha representa o segundo cilindro, externo, de raio $R_{2}$ e velocidade de rotação $w_{2}$ (Figura 5.1(b)). Neste trabalho é simulado o caso em que o cilindro interno está girando em sentido anti-horário e o cilindro externo está parado.

Os parâmetros da simulação são

- $\Omega=[0,1,875] \times[0,1,875]$

- $\rho=1,0 \mathrm{~g} / \mathrm{cm}^{3}, \mu=0,01 \mathrm{~g} / \mathrm{cms}$

- $R_{1}=0,15625 \mathrm{~cm}, R_{2}=0,65625 \mathrm{~cm}$, gap $=0,5 \mathrm{~cm}, R_{2} / R_{1}=4,2$

- $w_{1}=2,0 \mathrm{rad} / \mathrm{s}, w_{2}=0,0 \mathrm{rad} / \mathrm{s}$

- Condição inicial: $\mathbf{u}(\mathbf{x}, 0)=(0,0)$

- Condições de contorno no límite externo euleriano para a velocidade é Dirichlet homogênea

- As condições de contorno para as variáveis auxiliares são as dadas na Seção B.2 do Apêndice B.

- $T_{a}=\frac{\rho R_{1} w_{1} \text { gap }}{\mu}=15,625$. 
Simulações foram feitas para três diferentes tamanhos das malhas eulerianas e lagrangianas. Na Tabela 5.3, apresentam-se as descrições associadas às malhas euleriana e lagrangianas, onde: npl1 e npl2 são a quantidade de pontos lagrangianos para o primeiro e segundo cilindro, respectivamente, e NCgap é a quantidade de células eulerianas de separação entre os cilindros.

\begin{tabular}{|l|l|l|l|c|l|}
\hline$N_{x}=N_{y}$ & npl1 & npl2 & $\Delta x$ & NCgap & $\Delta t$ \\
\hline 60 & 64 & 264 & 0,031250 & 16 & {$[1,0 \mathrm{E}-5,1,0 \mathrm{E}-3]$} \\
120 & 128 & 528 & 0,015625 & 32 & {$[1,0 \mathrm{E}-5,1,0 \mathrm{E}-4]$} \\
240 & 256 & 1056 & 0,007812 & 64 & {$[1,0 \mathrm{E}-5,1,0 \mathrm{E}-4]$} \\
\hline
\end{tabular}

Tabela 5.3: Escoamento de Couette circular: tamanho das malhas eulerianas e lagrangianas

A condição de contorno de Dirichlet homogênea para a velocidade é usada para guardar a relação com a velocidade de rotação nula do cilindro externo. O critério para determinar se o regime estacionário foi atingido é baseado em [31]:

$$
\left\|\frac{\partial(u, v)}{\partial t}\right\|_{\max }<\frac{\epsilon}{N_{x} N_{y}}
$$

onde o valor de $\epsilon$ é uma constante, e neste caso adotou-se o valor de 0,001.

É computada, na região anular dos cilindros concêntricos, a norma máxima do erro entre a solução numérica dada pelo MFI/MFV e a solução exata , equação (5.12), assim como a razão dos erros para malhas $N \times N$ e $2 N \times 2 N$. Os resultados da Tabela 5.4 mostram uma convergência de primeira ordem. Esses resultados eram esperados, pois o Método de Fronteira Imersa é um método de primeira ordem, conforme discutido em [1], [8], [28], entre outros.

\begin{tabular}{|l|l|l|}
\hline $\mathrm{N}$ & $\left\|u-u_{a}\right\|_{\max }$ & razão \\
\hline 60 & $4,27602 \mathrm{e}-02$ & \\
120 & $2,29318 \mathrm{e}-02$ & 1,864668 \\
240 & $1,10977 \mathrm{e}-02$ & 2,06 \\
\hline
\end{tabular}

Tabela 5.4: Erros entre a solução exata e numérica para o escoamento de Couette utilizando $\mathrm{MFI} / \mathrm{MFV}$

Resultados do erro máximo entre a velocidade lagrangiana da interface e a velocidade do fluido na interface são reportados na Tabela 5.5 onde os resultados mostram um comportamento de segunda ordem. 


\begin{tabular}{|l|l|l|l|l|}
\hline $\mathrm{N}$ & $\left\|u k_{1}-u e k_{1}\right\|_{\max }$ & razão & $\left\|u k_{2}-u e k_{2}\right\|_{\max }$ & razão \\
\hline 60 & $3,25655 \mathrm{e}-03$ & & $1,81100 \mathrm{e}-04$ & \\
120 & $6,16178 \mathrm{e}-04$ & 5,28508 & $3,70857 \mathrm{e}-05$ & 4,8832839 \\
240 & $1,73282 \mathrm{e}-04$ & 4,8251266 & $7,71994 \mathrm{e}-06$ & 4,8038845 \\
\hline
\end{tabular}

Tabela 5.5: Erro do máximo entre a velocidade lagrangiana e a velocidade do fluido na respectiva malha lagrangiana obtidos através do MFV.

As Figuras 5.2 e 5.3 mostram o campo da pressão e as linhas de corrente, respectivamente, associadas ao escoamento de Couette circular.

Observa-se que a pressão é maior ao redor da interface correspondente ao cilindro externo do que aquela correspondente ao cilindro interno, e uma redução não linear da pressão pode ser observada na região anular. Tais comportamentos são qualitativamente consistentes.

As linhas de corrente da Figura 5.3 mostram três comportamentos importantes obtidos pela metodologia: (1) as linhas de corrente ao redor do cilindro interno seguem o sentido anti-horário de rotação deste cilindro tanto fora deste quanto no seu interior; (2) as linhas de corrente ao redor do cilindro externo são contra-rotativas o que reflete a recuperação da condição de contorno de não deslizamento modelada virtualmente sobre este cilindro; e (3) linhas de corrente ao redor da fronteira do domínio euleriano, $\gamma=\partial \Omega$, apresentam recirculações na vizinhançãs dos cantos deste domínio. Observa-se que o escoamento externo ao canal anular é induzido puramente pelo campo de força avaliado pelo MFV. É interessante perceber que células secundárias foram capturadas nos quatros cantos do domínio.

\subsection{Pêndulo imerso em um fluido}

Este problema consiste em um pêndulo imerso em um fluido contido em uma caixa fechada. Inicialmente o sistema fluido-pêndulo encontra-se em repouso com o pêndulo posicionado de modo a formar um ângulo $\theta>0$ com o eixo $-y$ (Figura 5.4). Aqui o pêndulo é representado utilizando-se um cilindro.

A solução analítica para este problema não é conhecida, embora o comportamento físico da dinâmica do pêndulo seja conhecida. A diferença deste problema com o do escoamento de Couette circular é que não se conhece a priori a velocidade do corpo imerso, 


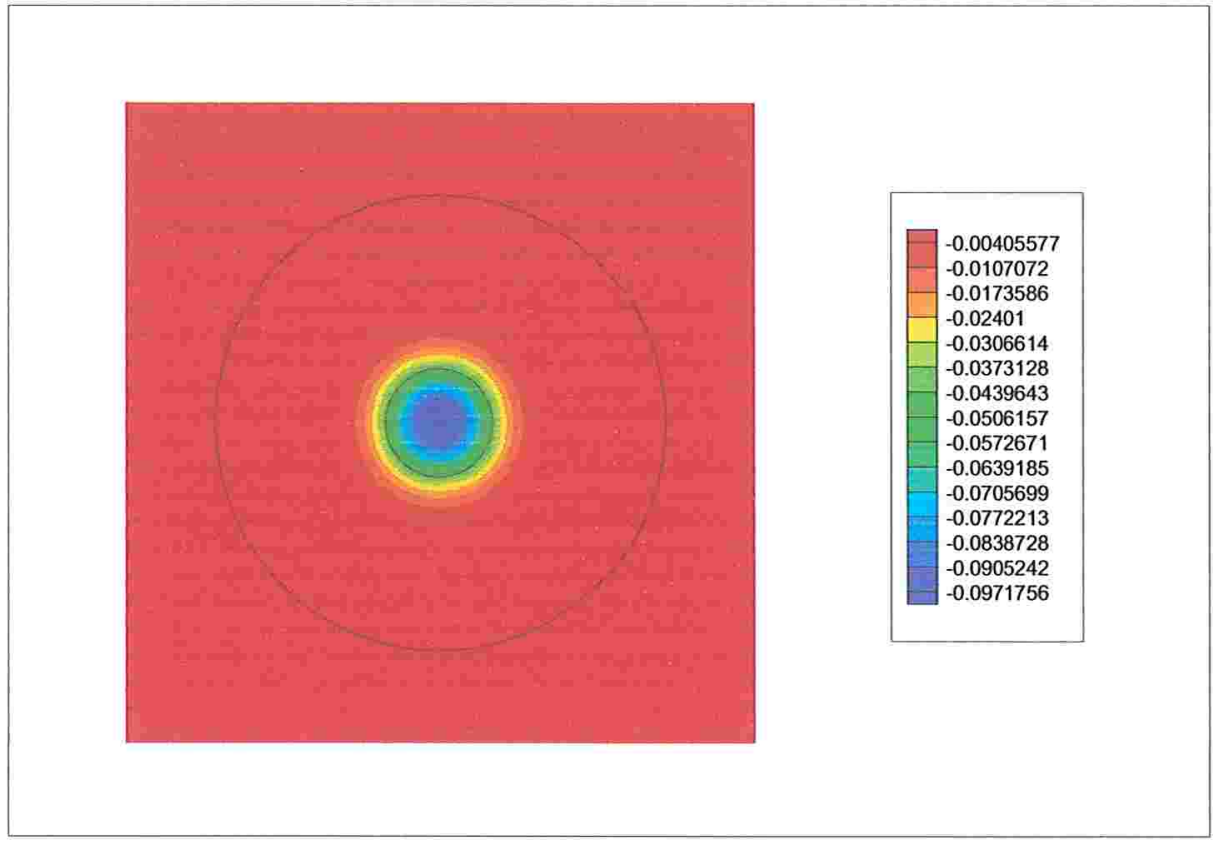

Figura 5.2: Escoamento de Couette circular: campo de pressão (malha $60 \times 60$ ).

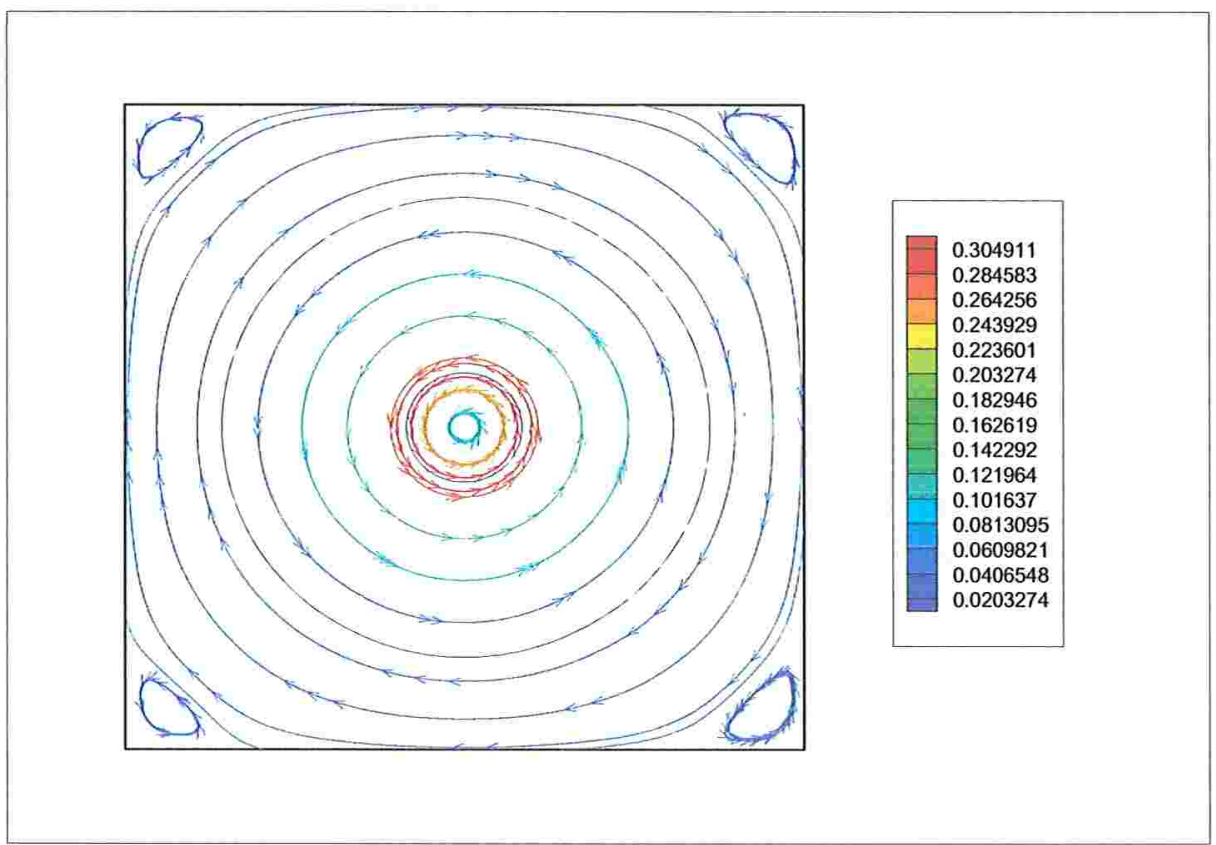

Figura 5.3: Escoamento de Couette circular: linhas de corrente (malha $60 \times 60$ ). 
esta é determinada no processo de integração das equações. Este problema, apesar de sua configuração geométrica simples, já caracteriza um problema de interação fluido-estrutura.

Para determinar a dinâmica do pêndulo no fluido deve-se integrar as equações associadas ao seu movimento. Para tal é necessário determinar o campo de forças total atuando sobre ele. Neste caso, além da força de gravidade e de empuxo tem-se a força fluidodinâmica atuando sobre ele.

Na Figura 5.4 é apresentado o diagrama de forças que atuam no pêndulo. Descompondose as forças nas direções radiais e tangencias ao movimento e usando o fato de que a trajetória do pêndulo é conhecida, tem-se que a resultante das componente das forças na direção radial sempre é nula, enquanto que na direção tangencial não necessariamente o é. Portanto, tem-se uma equação para o movimento tangencial devido à resultante das forças de ação nessa direção.

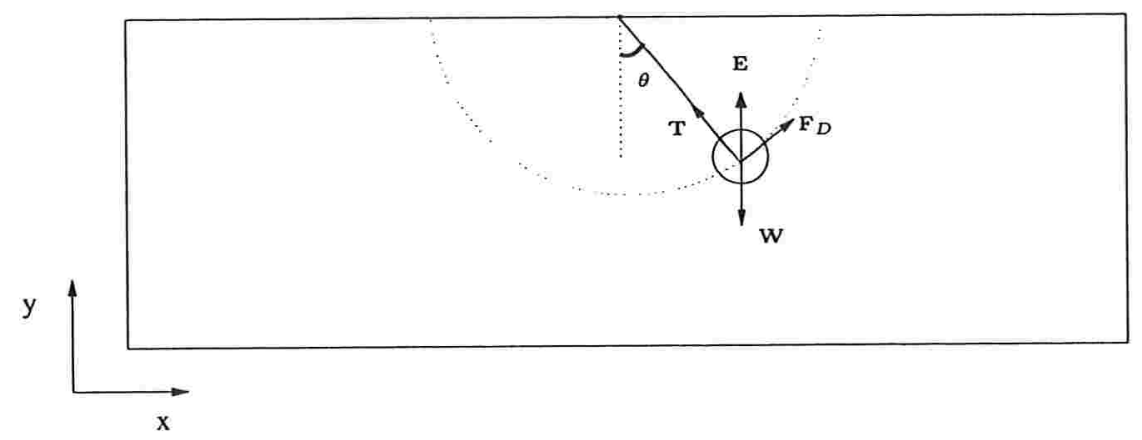

Figura 5.4: Pêndulo imerso em uma caixa contendo um fluido.

\section{Solução numérica via o MFI/MFV}

Para representar o pêndulo imerso num fluido é considerada uma malha lagrangiana cujos pontos são provenientes da discretização de uma circunferência de raio $R$. A corda que suspende o pêndulo não é considerada diretamente no fluido. A presença da corda é imposta assumindo-se que não haja movimento na direção radial.

$\mathrm{O}$ algoritmo pode ser resumido por:

- Dada a posição inicial do pêndulo $\theta^{0}$, calcula-se a força resultante sobre ele, levando en conta o peso, o empuxo e o arrasto; 
- Com esta força resultante projetada na direção tangencial da sua trajetória, calculase sua aceleração angular;

- Calcula-se sua velocidade angular e transporta-se o pêndulo para nova posição $\theta^{1}$;

- Com a velocidade angular determinada $\dot{\theta}^{0}$ e com os campos de velocidade e pressão eulerianos calcula-se a força lagrangiana pelo MFV;

- Resolvem-se as equações de Navier-Stokes para a nova posição $\theta^{1}$ levando-se em conta a força lagrangiana do passo precedente (força explícita);

- Conhecidos os campos de velocidade e pressão eulerianos recalcula-se o novo campo de forças lagrangiano, transporta-se o pêndulo e reinicia-se o processo.

Os seguintes parâmetros são considerados para o pêndulo e para o fluido:

- Sistema de unidades CGS

- $\Omega=[0,0,6,0] \times[0,0,2,0]$ (dimensão do domínio)

- $\rho=1,0 \mathrm{~g} / \mathrm{cm}^{3}, \mu=5,0 E-3 \mathrm{~g} / \mathrm{cms}$

- $R=0,125 \mathrm{~cm}$

- $\rho_{c}=3,0 \mathrm{~g} / \mathrm{cm}^{3}$

- $L_{p}=1,0 \mathrm{~cm}$

- $\mathrm{g}=(0,0,-980,67) \mathrm{cm} / \mathrm{s}^{2}$

- Posição inicial do pendulo $=(3,98,1,83) \mathrm{cm}\left(\theta^{0}=1.4 \mathrm{rad}\right)$

- Condição inicial: $\mathbf{u}(\mathbf{x}, 0)=(0,0) \mathrm{cm} / \mathrm{s}$

- Condições de contorno para a fronteira externa para a velocidade é Dirichlet homogênea

- As condições de contorno para as variáveis associadas são as dadas na Seção B.2 do Apêndice B. 
A simulação numérica foi feita numa malha euleriana de tamanho $N_{x} \times N_{y}$, onde: $N_{x}=$ 1536 e $N_{y}=512\left(\Delta x=\Delta y=3,90625 \times 10^{-3}\right)$ e considerou-se $n p l=816$.

A Figura 5.5 mostra a história temporal do deslocamento angular, $\theta$, que o pêndulo faz com o eixo $y$. Este valor decresce conforme passa o tempo. Observa-se que o módulo das amplitudes quando o pêndulo movimenta também decresce. Um comportamento similar para a velocidade angular é observado na Figura 5.6. Observa-se que o período das oscilações se mantém praticamente constante, como prevê a teoria de sistemas dinâmico não amortecidos.

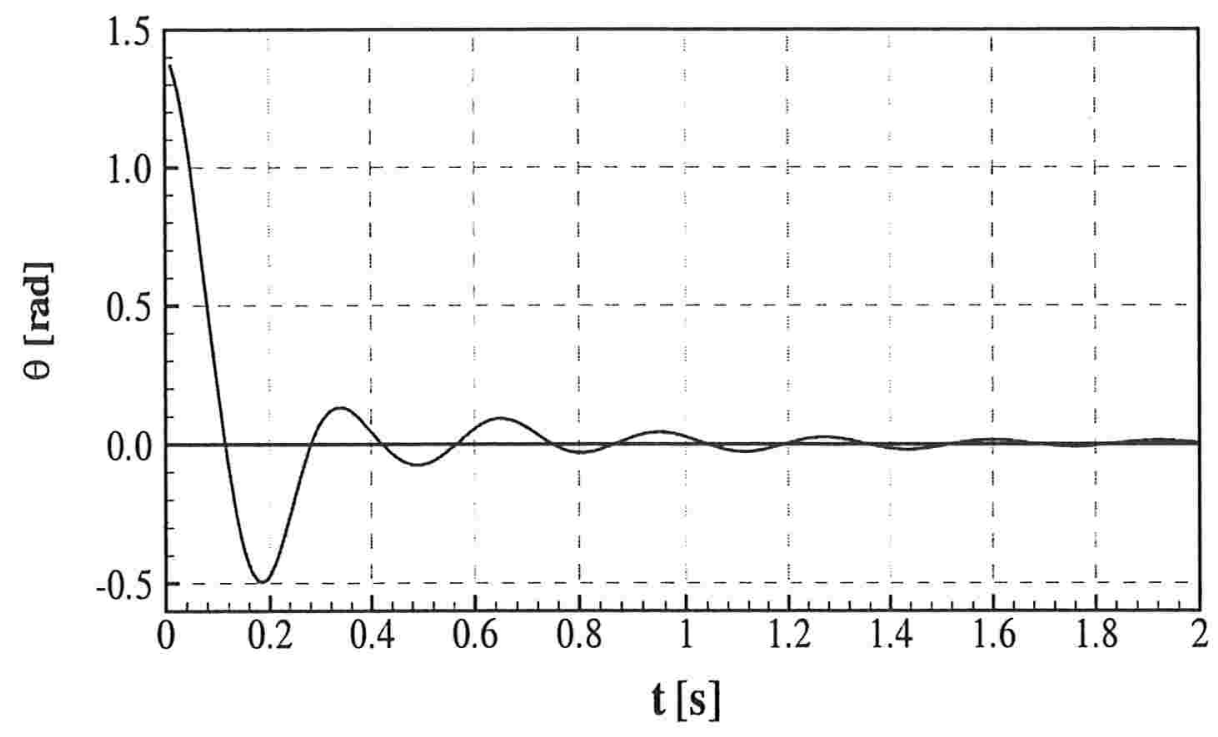

Figura 5.5: Pêndulo imerso num fluido: história temporal do ângulo $\theta$ (rad).

Na Figura 5.7 e 5.8 mostram-se o valor do número de Reynolds definido com $\operatorname{Re}_{D}=$ $\frac{U_{c} D}{\nu}$, onde $U_{c}$ é a velocidade do cilindro que depende do tempo, e o módulo da força fluidodinâmica atuando no corpo $\left(\mathbf{F}_{\mathrm{D}}\right)$, respectivamente. O máximo número de Re é aproximadamente 800 e é atingido para $\theta=0$ no primeiro ciclo do pêndulo. Ambas figuras mostram que estas grandezas decrescem conforme passa o tempo.

Na Figura 5.9 mostram-se o valor absoluto das diferenças entre a força total $\mathbf{F}_{\mathrm{T}}$ que atua sobre o cilindro (levando-se em conta o arrasto $\mathbf{F}_{\mathrm{D}}$, o peso $\mathbf{W}$ e o empuxo $\mathbf{E}$ ) e a força peso menos o empuxo, $\mathbf{F}_{\mathrm{WE}}$ e a força de arrasto, $\mathbf{F}_{\mathrm{D}}$. Percebe-se que a força $\mathbf{F}_{\mathrm{WE}}$ (diferença entre peso e empuxo) é predominante sobre a força de arrasto na fase inicial do problema. À medida que o tempo passa o arrasto assume uma magnitude similar ao peso 


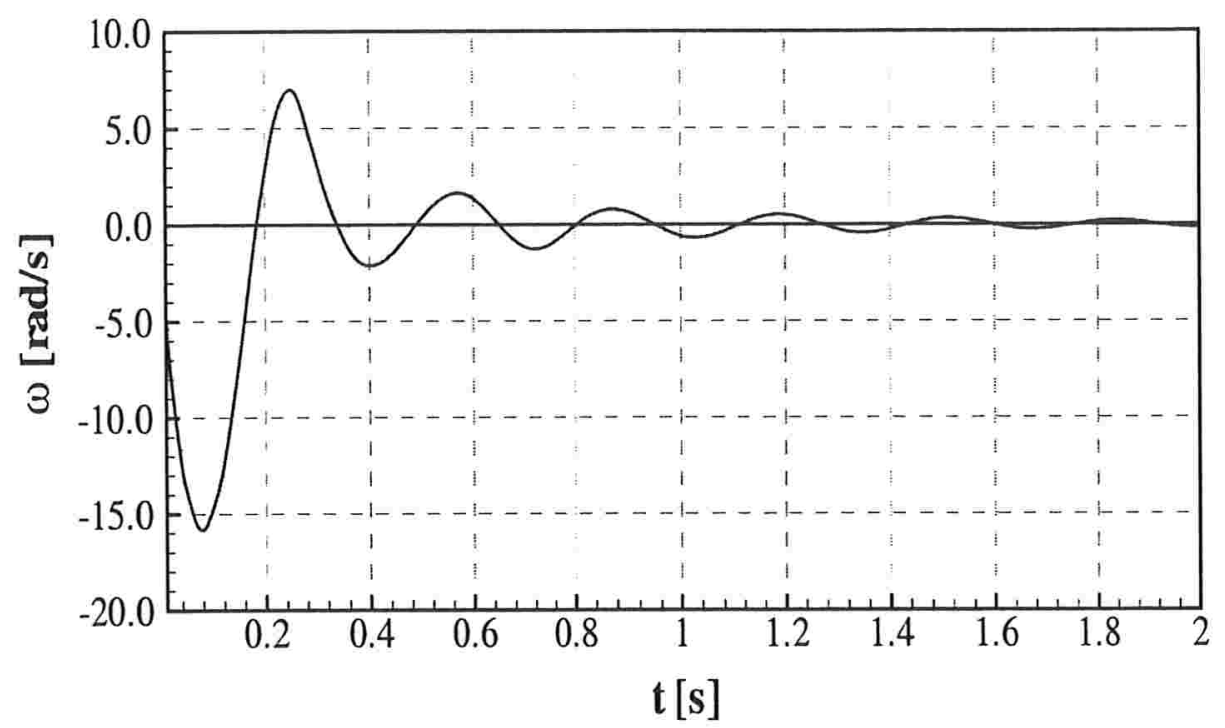

Figura 5.6: Pêndulo imerso num fluido: história temporal da velocidade angular $w$.

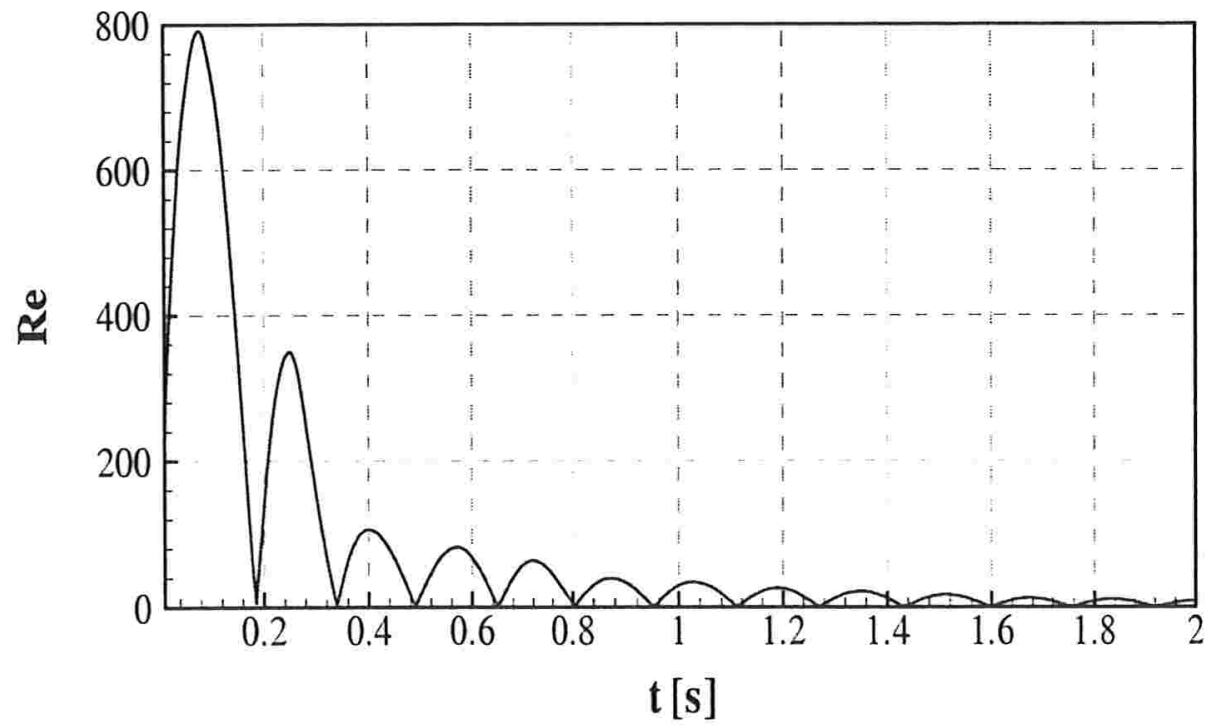

Figura 5.7: Pêndulo imerso em um fluido incompressível: história temporal do número de Reynolds. 


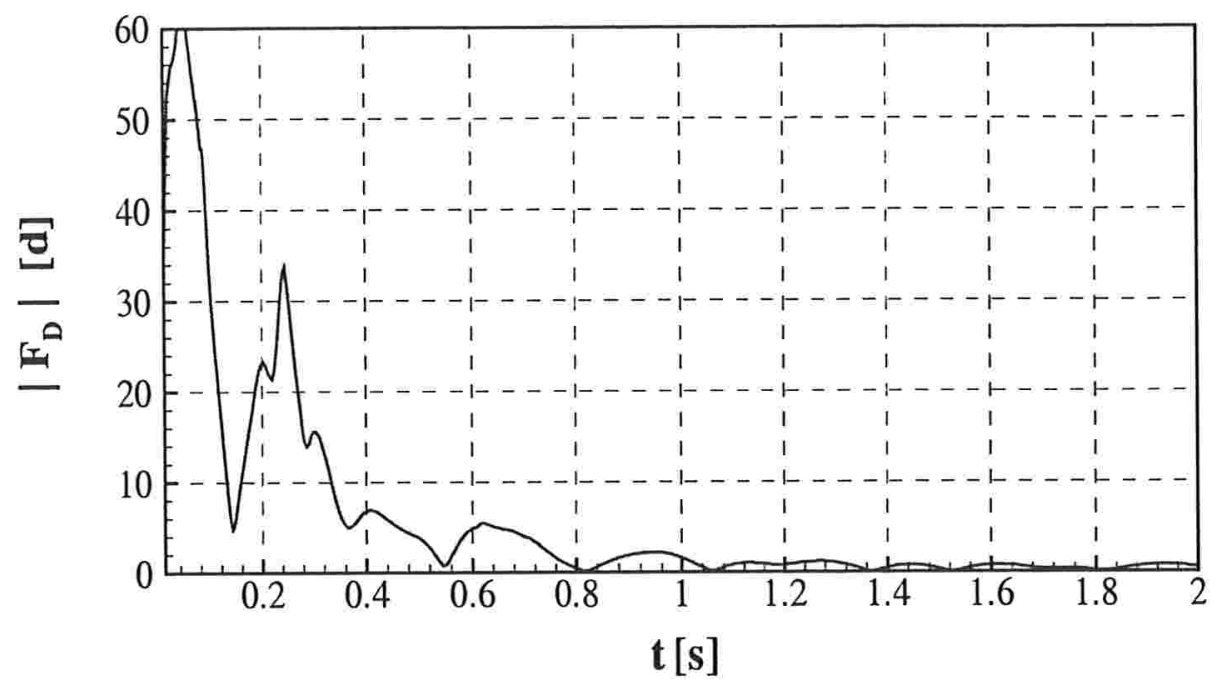

Figura 5.8: Módulo da força total fluidodinâmica $\mathbf{F}_{\mathrm{D}}$ atuando no corpo (dinas).

menos o empuxo. Este resultado é fisicamente consistente, uma vez que o amortecimento viscoso é menor que as forças aceleradoras. Isto explica o porque do elevado número de ciclos necessários para levar o cilindro ao repouso.

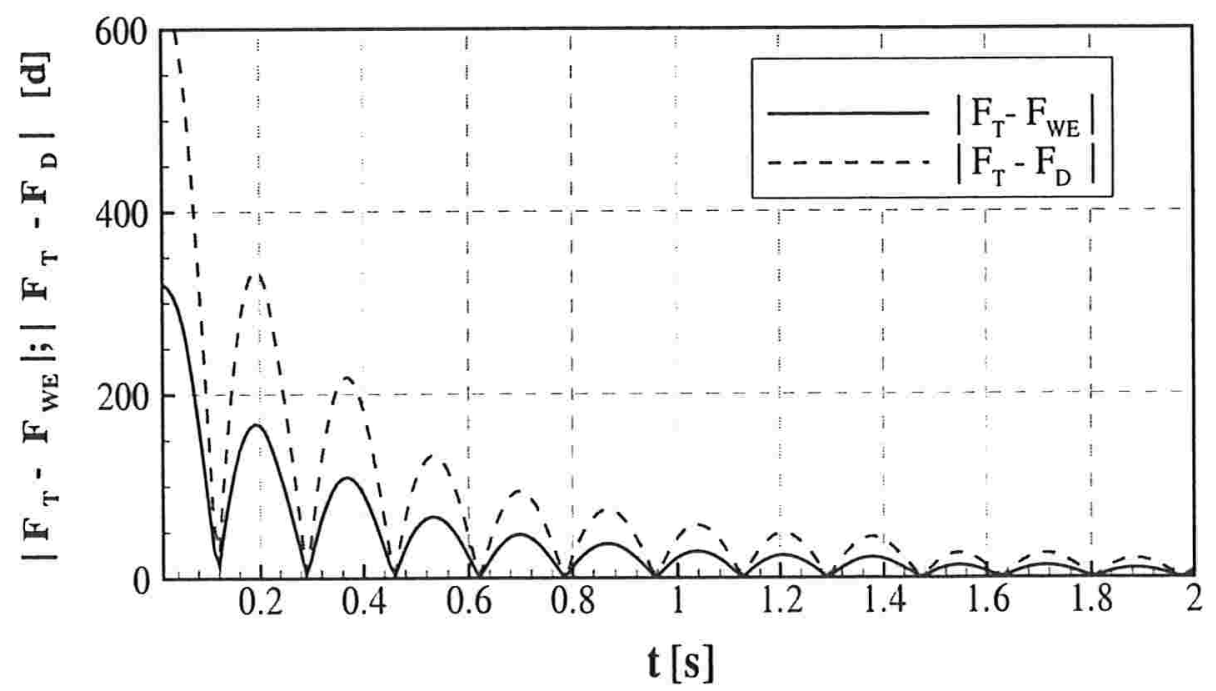

Figura 5.9: Módulo da diferenca entre a força total atuando na direção do movimento e as contribuições $\mathbf{F}_{\mathrm{WE}}$ e $\mathbf{F}_{\mathrm{D}}$. 


\subsection{Canal-cavidade com válvulas cilíndricas}

No trabalho de Arruda [1] foi apresentada a simulação numérica, utilizando-se MFI/MFV, do escoamento de um fluido no interior de um conjunto canal-cavidade onde o escoamento é originado do movimento periódico prescrito do fundo da cavidade. Os resultados obtidos por Arruda [1] foram muito promissores, demonstrando que este tipo de modelagem pode ser aplicado a problemas práticos de alta complexidade. Nesse contexto, o presente trabalho é uma extensão da aplicação da metodologia MFI/MFV para um estudo preliminar de um modelo simplificado de um dispositivo de bombeamento.

O modelo simplificado do dispositivo de bombeamento está representado por um conjunto canal-cavidade com duas contrações, localizadas na entrada e na saída do canal, e dois cilindros, como apresentado na Figura 5.10. O fundo da cavidade se movimenta com velocidade periódica imposta $V(t)$. O movimento dos cilindros é restrito à direção horizontal, e é promovido exclusivamente pelo movimento do fluido.

O escoamento neste modelo se deve à movimentação do fundo da cavidade. No entanto, a dinâmica do escoamento é influenciada pela interação do fluido com os cilindros.

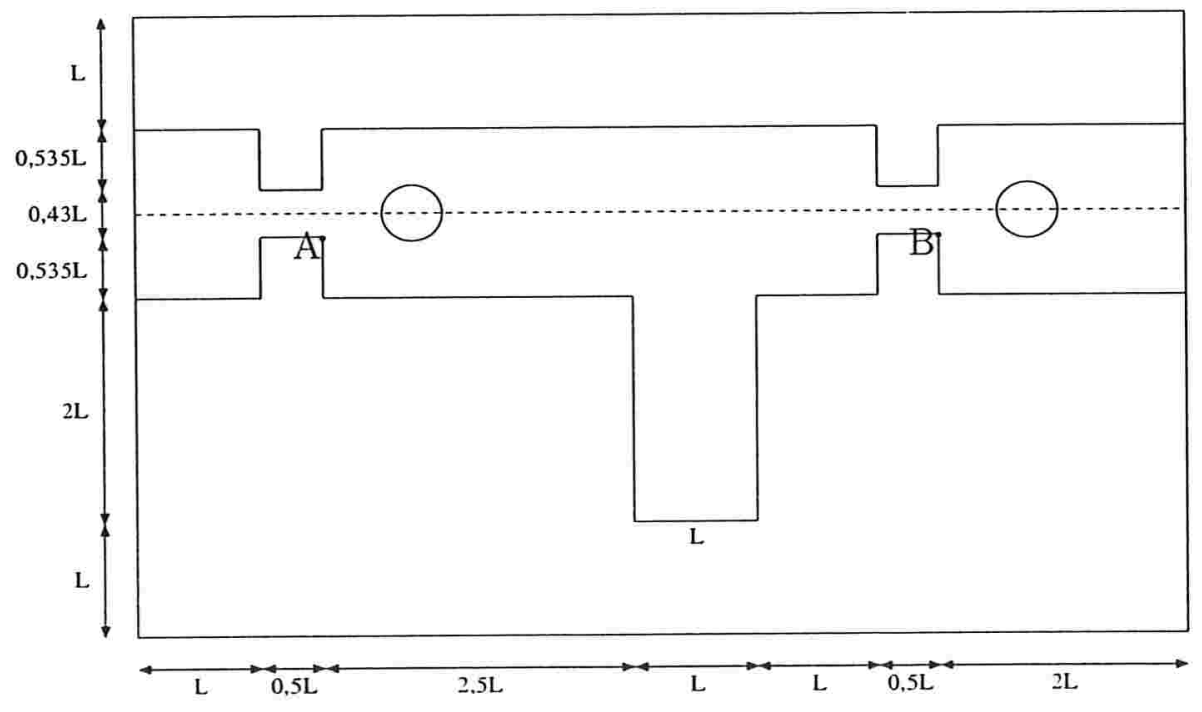

Figura 5.10: Representação geométrica do conjunto canal-cavidade contendo dois cilindros imersos (dimensões em função do comprimento do pistão, L). Os pontos A e B são pontos de referência a serem usadas com o cilindro do lado esquerdo e do lado direito, respectivamente. 
A seguir apresentam-se as condições para o problema considerado e algumas experiências desenvolvidas com o intuito de conhecer mais sobre o comportamento da modelagem matemática adotada.

\subsubsection{Descrição das condições do problema}

Para esta simulação foram especificados os seguintes parâmetros para o sistema fluidoestruturas rígidas:

- Sistema de unidades: MKS

- Domínio $\Omega=[0 ; 0,2125] \times[0 ; 0,1375]$

- Densidade do fluido, $\rho=1,0 \mathrm{~kg} / \mathrm{m}^{3}$, e viscosidade $\mu=1,7 \times 10^{-5} \mathrm{~kg} / \mathrm{ms}$

- Velocidade do pistão é dada por:

$$
V(t)=V_{0} \sin (20 \pi t)[\mathrm{m} / \mathrm{s}],
$$

onde $V_{0}$ é a amplitude para a velocidade do fundo da cavidade (pistão) sendo adotado o valor de $0,9 \mathrm{~m} / \mathrm{s}$. A frequência do movimento do pistão é $10 \mathrm{~Hz}$ e o período é $0,1 \mathrm{~s}$. Na Figura 5.11 apresenta-se o gráfico da distribuição da velocidade do pistão em função do tempo.

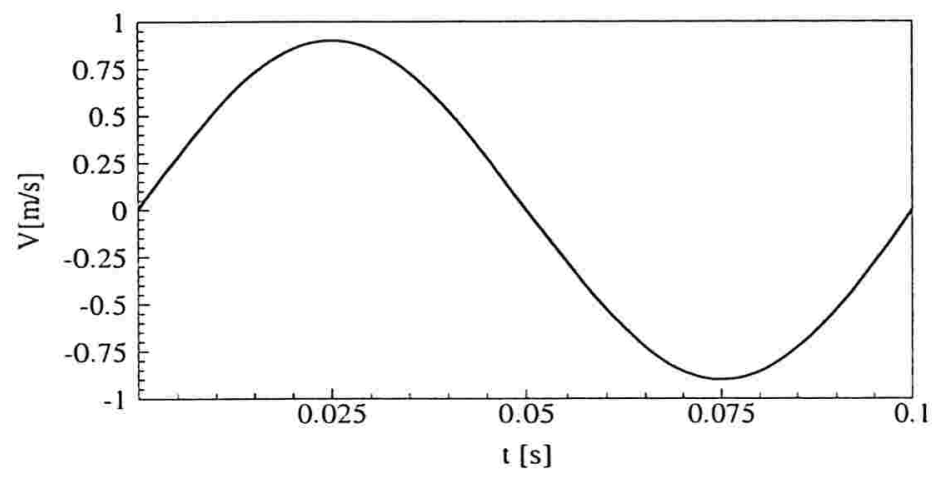

Figura 5.11: Velocidade periódica para o fundo móvel.

- O número de Reynolds foi calculado como $R e=\frac{\rho U(t) H(t)}{\mu}$, onde $U(t)$ é a velocidade do fundo móvel no tempo $t$ e $H(t)$ é a altura da cavidade no tempo t. A Figura 5.12 apresenta o perfil do número de Reynols para a cavidade com fundo móvel. 


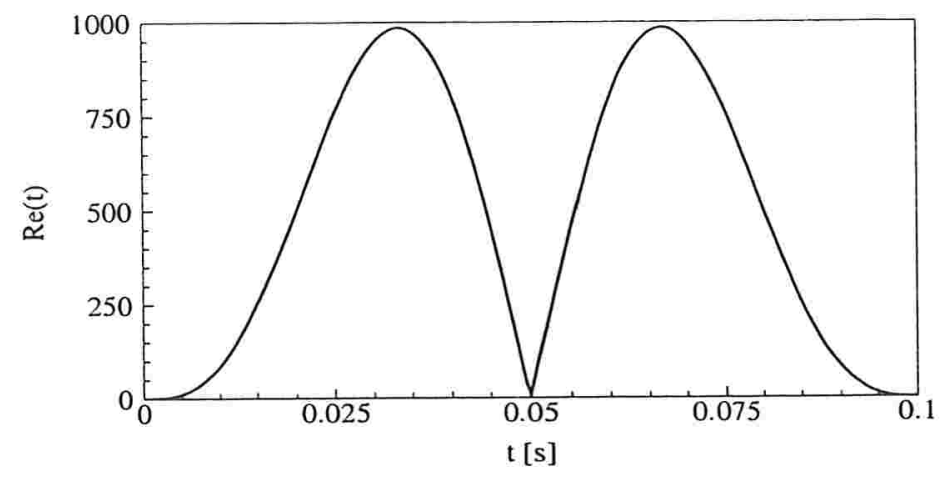

Figura 5.12: História temporal do número de Reynolds para a cavidade com fundo móvel periódica.

- A modelagem da turbulência foi realizada empregando o Modelo de Smagorinsky, onde o valor da constante de Smagorinsky considerado foi $C_{s}=0,3$.

- As dimensões do canal-cavidade são mostradas na Figura 5.10 e estas são dadas em função do comprimento da parede do fundo móvel, L. Neste caso o valor adotado para o comprimento do fundo móvel é $L=0,025 \mathrm{~m}$.

- Os raios dos cilindros são iguais a $6,2 \times 10^{-3} \mathrm{~m}$. Com este raio os cilindros formam um ângulo $\alpha=60^{\circ}$ no momento de contato com as paredes das contrações como é ilutrada na Figura 5.13

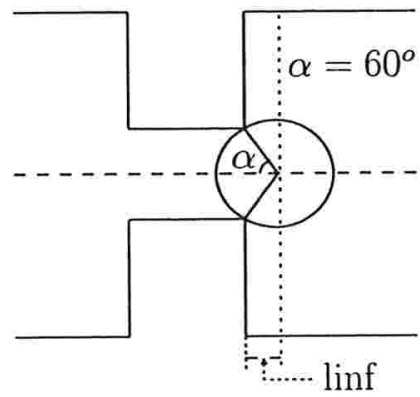

Figura 5.13: Ângulo formado pelo contato do cilindro com o vértice da contração e a linha horizontal que passa pelo centro de massa. O comprimento linf é o valor mínimo da coordenada $x$, do centro de massa do cilindro com respeito ao vértice da contração

- Os cilindros são considerados de mesma densidade 


\subsubsection{Solução numérica através do MFI/MFV}

Uma malha regular euleriana de tamanho $N_{x} \times N_{y}$ com $N_{x}=340$ e $N_{y}=220$ é usada. O espaçamento da malha é $\Delta x=\Delta y=6,25 \times 10^{-4} \mathrm{~m}$. Três malhas lagrangianas são usadas: a primeira, define o contorno do conjunto canal-cavidade; a segunda, define o contorno do cilindro esquerdo e a última o contorno do cilindro direito. Os números de pontos lagrangianos em cada malha lagrangiana são: 2048 para malha do canal-cavidade, 124 pontos lagrangianos para cada cilindro. O espaçamento entre os pontos lagrangianos é tal que existe pelo menos dois pontos lagrangianos por célula computacional euleriana.

As condições iniciais para o sistema são: velocidade e pressão nulas, fundo móvel da cavidade localizado na parte inferior, enquanto que a posição ínicial dos cilindros é um parâmetro a ser definido para cada experiência.

As condiçoes de contorno para a velocidade do fluido são do tipo Neumann homogênea (condição de contorno para as variáveis auxiliares do passo de projeção estão indicadas no Apêndice B). As estruturas rígidas na metodologia do MFI/MFV não precisam de condição de contorno.

A posição do pistão é obtida através da integração da equação:

$$
\frac{d Y_{k}}{d t}=V(t)
$$

onde $Y_{k}$ indica a posição de cada ponto $k$ da cavidade e $V(t)$ é a velocidade especificada em (5.13).

Para determinar a posição dos cilindros deve-se resolver as equações associadas ao centro de massa, equações (3.17) e (3.19).

\subsubsection{Experiência 1: movimento horizontal livre dos cilindros}

Nesta experiência os cilindros têm movimentos restritos à horizontal, sendo limitados à suas esquerdas pelos estrangulamentos e podem se mover para a direita livremente, sob a influência do escoamento.

Experiências computacionais foram realizadas considerando os cilindros com densidades iguais e diversas posições iniciais. Dois valores para a densidade dos cilindros são considerados, sendo que para cada um deles os cilindros foram localizados em três diferentes posições iniciais. 
Nas Figuras 5.14 e 5.15 apresentam-se os comportamentos temporais dos centros de massa dos cilindros. Na Figura 5.14 mostram-se os comportamentos temporais para três posições iniciais arbitrárias dos centros de massa dos cilindros, em função das coordenadas relativas aos pontos A e B (Figura 5.10), para cilindros com densidades iguais a $0,5 \mathrm{~kg} / \mathrm{m}^{3}$. $\mathrm{O}$ cilindro localizado à direita do canal, com centro de massa localizado $\mathrm{em} \mathrm{xcm} 2$, tem a mesma posição inicial para os três casos apresentados nesta figura. Por outro lado, o cilindro localizado no lado esquerdo assume três posições iniciais para o centro de massa (xcm1), cada vez mais afastadas das estrangulações, caracterizando três experiências distintas. A linha horizontal na Figura 5.14 denotada por linf corresponde à posição limite inferior para o deslocamento dos centros de massa (mostrada na Figura 5.13).

Na Figura 5.14 (a) o cilindro da esquerda se encontra inicialmente de $\mathrm{A}$ em ( $\mathrm{xcm} 1$, $\mathrm{ycm} 1)=(0,009,0,005)[\mathrm{m}]$ enquanto o cilindro 2 se encontra em $(\mathrm{xcm} 2, \mathrm{ycm} 2)=(0,006$, $0,005)[\mathrm{m}]$ de B. Percebe-se que, com a ascensão do pistão, no primeiro ciclo, o cilindro 1 (da esquerda) se desloca para a esquerda e o seu centro de massa ultrapassa o limite inferior. Isto significa que o cilindro sólido penetrou pelas paredes do estrangulamento, mostrando uma inconsistência física que aparece devido ao fato que a metodologia da fronteira imersa, como aplicada no presente trabalho, não modela corretamente o choque do cilindro contra uma parede. Por outro lado, a variável relativa a xcm2 mostra que o cilindro 2 se desloca livremente para a direita. Com o passar do tempo ambos os cilindros oscilam em função do movimento do pistão. No entanto, o primeiro cilindro se afasta do respectivo estrangulamento continuamente.

Na Figura 5.14 (b) o cilindro 1 é colocado inicialmente em $(\mathrm{xcm} 1, \mathrm{ycm} 1)=(0,017$, $0,005)[\mathrm{m}]$ e o cilindro 2 é colocado inicialmente na mesma posição do caso precedente. Os cilindros, neste caso, se movimentam oscilando, mostrando uma leve ascenção média, o que pode ser produzido que os cilindros estão se deslocando para a direita. Assim, tem-se uma maior estabilidade da movimentação quando comparada com os casos precedentes. Observa-se que os movimentos dos cilindros não estão em fase com o pistão, devido às suas inércias.

Na Figura 5.14 (c) o cilindro 1 é liberado em outra posição $(\mathrm{xcm} 1, \mathrm{ycm} 1)=(0,031$, $0.005)[\mathrm{m}]$ e o cilindro 2 na mesma posição anterior. Novamente pode-se observar uma movimentação mais estável dos cilindros, se comparada com o caso da Figura 5.14 (a).

Nas figuras 5.15 apresenta-se o movimento dos centros de massa para uma densidade do cilindro de 1,5 (maior que a do fluido). Observam-se comportamentos similares aos da figura anterior, com leves diferenças em relação à amplitude dos respectivos deslocamen- 


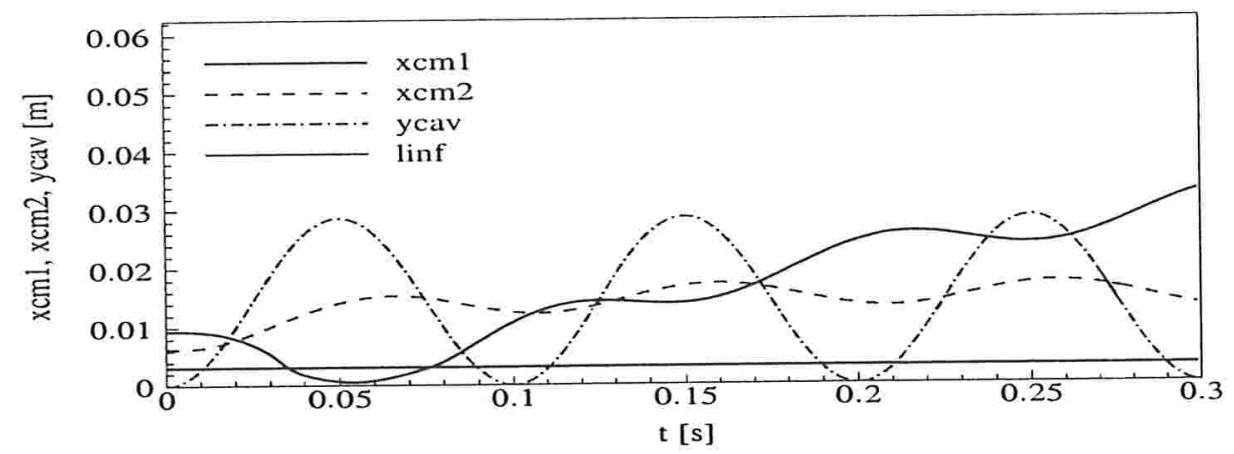

(a) $\mathbf{X}_{c m 1}=(0,009,0,005)$ e $\mathbf{X}_{c m 2}=(0,006,0,005)$

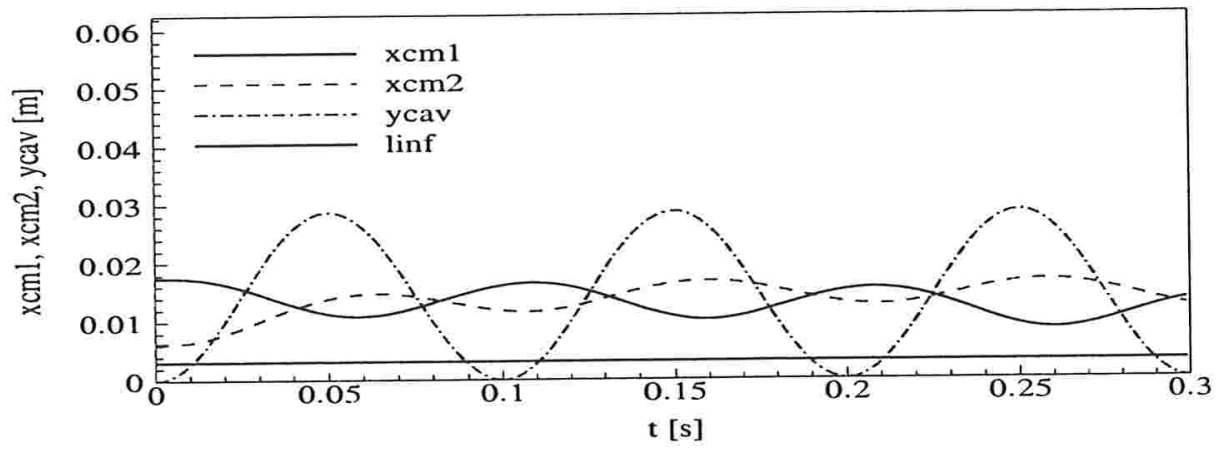

(b) $\mathbf{X}_{c m 1}=(0.017,0,005)$ e $\mathbf{X}_{c m 2}=(0,006,0,005)$

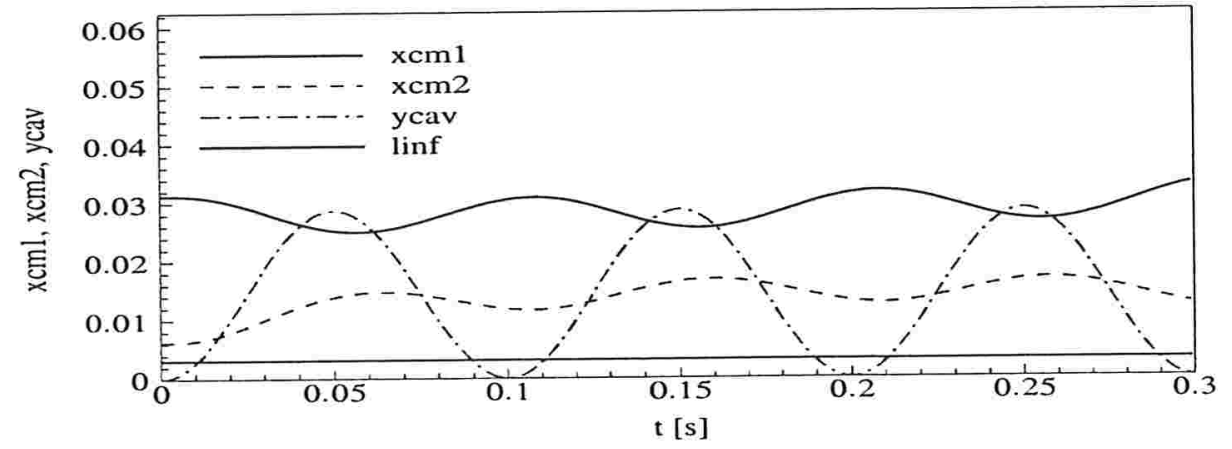

(c) $\mathbf{X}_{c m 1}=(0,031,0,005)$ e $\mathbf{X}_{c m 2}=(0,006,0,005)$

Figura 5.14: Movimento livre dos cilindos: abscissas relativas do centro de massa dos cilindros ( $\mathrm{xcm} 1$ e $\mathrm{xcm} 2$ ) aos pontos $\mathrm{A}$ e $\mathrm{B}$ das contrações; altura do fundo móvel da cavidade, ycav; valor mínimo para a abscissa dos cilindros, linf. $\left(\rho_{c}=0,5 \rho\right.$ e $v_{0}=$ $0,9 \mathrm{~m} / \mathrm{s})$. 
tos. Percebe-se na Figura 5.15 (a) que o cilindro de centro de massa xcm1, inicialmente ultrapassa os limites de referência (sem sentido físico). Isto explica-se pelo fato que o escoamento está em fase de desenvolvimento, onde o campo de forças que representa a presença da interface não está ainda adequadamente quantificada. A posição inicial dos cilindros influencia na dinâmica do escoamento.

Os resultados mostrados indicam a necessidade de melhores especificações que permitam um melhor controle dos corpos móveis.

\subsubsection{Experiência 2: movimento dos cilindro limitados mecani- camente}

Neste caso, os cilindros são limitados mecanicamente através de batentes mecânicas. Na Figura 5.16 apresenta-se uma vista de todo o domínio ilustrando a localização dos cilindros em relação aos batentes e em relação aos estrangulamentos (pontos A e B). Estes batentes são modelados matemáticamente por meio de imposição de condições locais nulas para a velocidade do centro de massa, isto é $\mathrm{V}_{c}=0$.

A imposição da velocidade nula para o centro de massa é aplicada quando a posição deste atinge o limite especificado. Nesse caso, tal especificação é feita através de limitadores para as abscissas dos centros de massa. Cada cilindro tem associados limitantes, e na modelagem adotada considera-se que os espaçamentos entre eles são iguais para os dois cilindros.

Denota-se por LimI e LimS os limitantes inferior e superior para o centro de massa dos cilindros 1 e 2, relativos aos pontos A e B (Figura 5.16). Usando-se tais limitantes, as abscissas dos centros de massa dos cilindros (relativos aos pontos A e B) satisfazem:

$$
\operatorname{LimI} \leq \mathrm{xcm} 1 \leq \operatorname{LimS}, \quad \operatorname{LimI} \leq \mathrm{xcm} 2 \leq \operatorname{LimS} .
$$

Os seguintes valores são considerados para os limitantes (espaçamento entre os limitantes igual a duas vezes o raio dos cilindros)

$$
\operatorname{Lim} I=0,012 m, \quad \operatorname{Lim} S=\operatorname{Lim} I+2 R .
$$

A Figura 5.17 apresenta as posições dos centros de massa dos cilindros 1 e 2 (xcm1 e xcm2), em relação aos pontos A e B (Figura 5.16). Assim, na Figura 5.17 mostram-se as posições dos centros de massa de ambos os cilindros, cujos valores estão limitados pelos valores LimI e LimS, representados nas figuras pelas linhas horizontal inferior e horizontal 


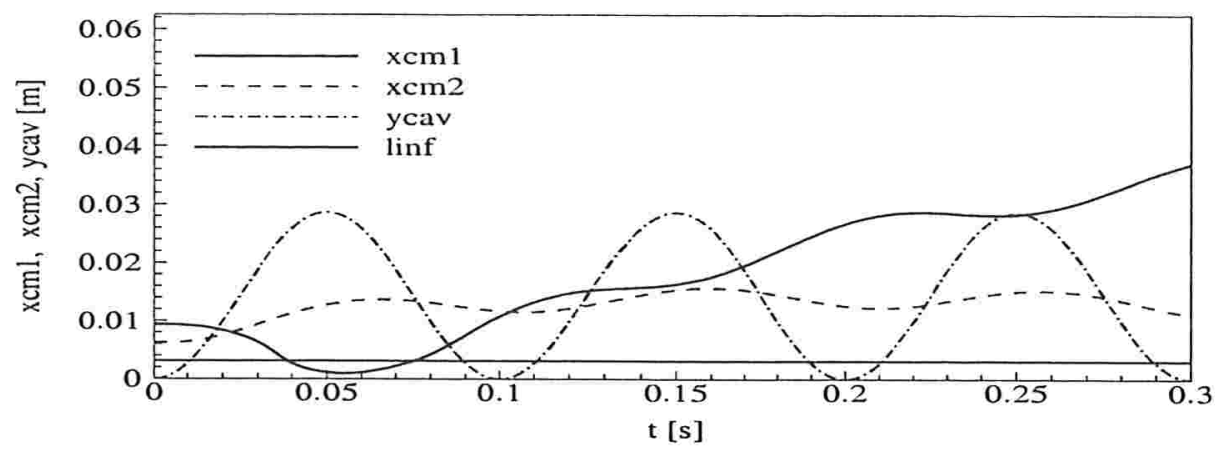

(a) $\mathbf{X}_{c m 1}=(0,009,0,005)$ e $\mathbf{X}_{c m 2}=(0,006,0,005)$

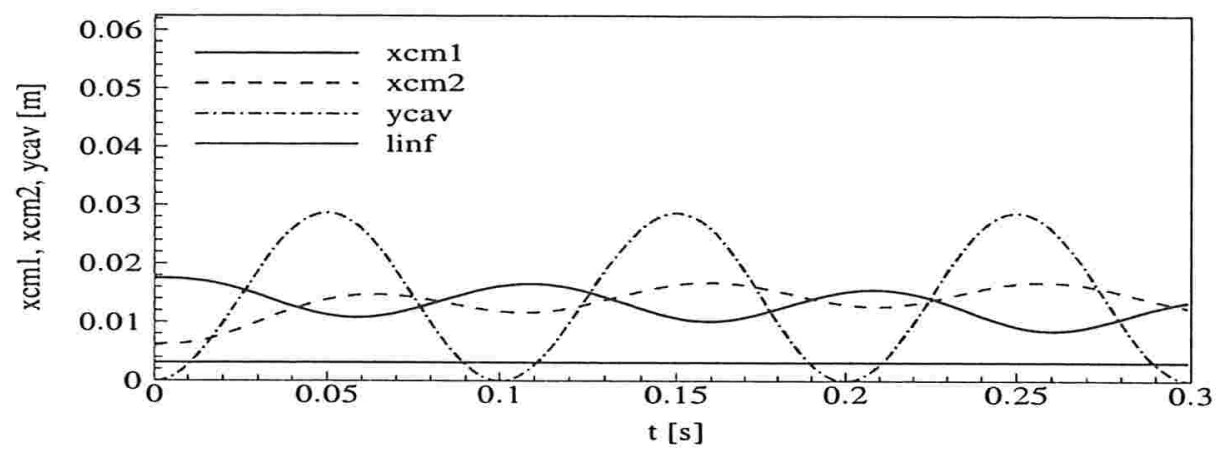

(b) $\mathrm{X}_{c m 1}=(0.017,0,005)$ e $\mathrm{X}_{c m 2}=(0,006,0,005)$

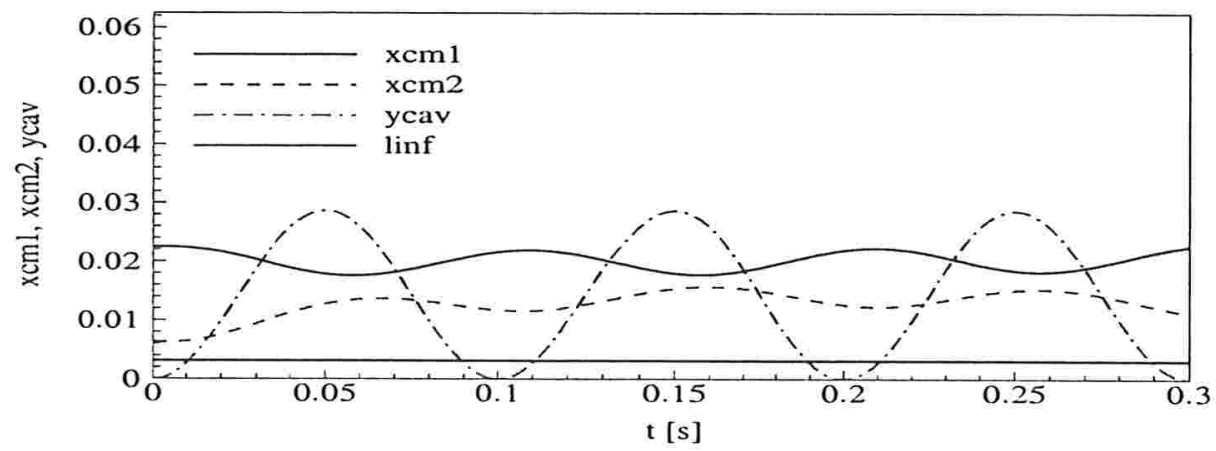

(c) $\mathrm{X}_{c m 1}=(0.021,0,005)$ e $\mathrm{X}_{c m 2}=(0,006,0.005)$

Figura 5.15: Movimento livre dos cilindos: abscissas relativas do centro de massa dos cilindros ( $\mathrm{xcm} 1$ e $\mathrm{xcm} 2)$ aos pontos A e B das contrações; altura do fundo móvel da cavidade, ycav; valor mínimo para a abscissa dos cilindros, linf. $\left(\rho_{c}=1,5 \rho\right.$ e $v_{0}=$ $0,9 \mathrm{~m} / \mathrm{s})$. 


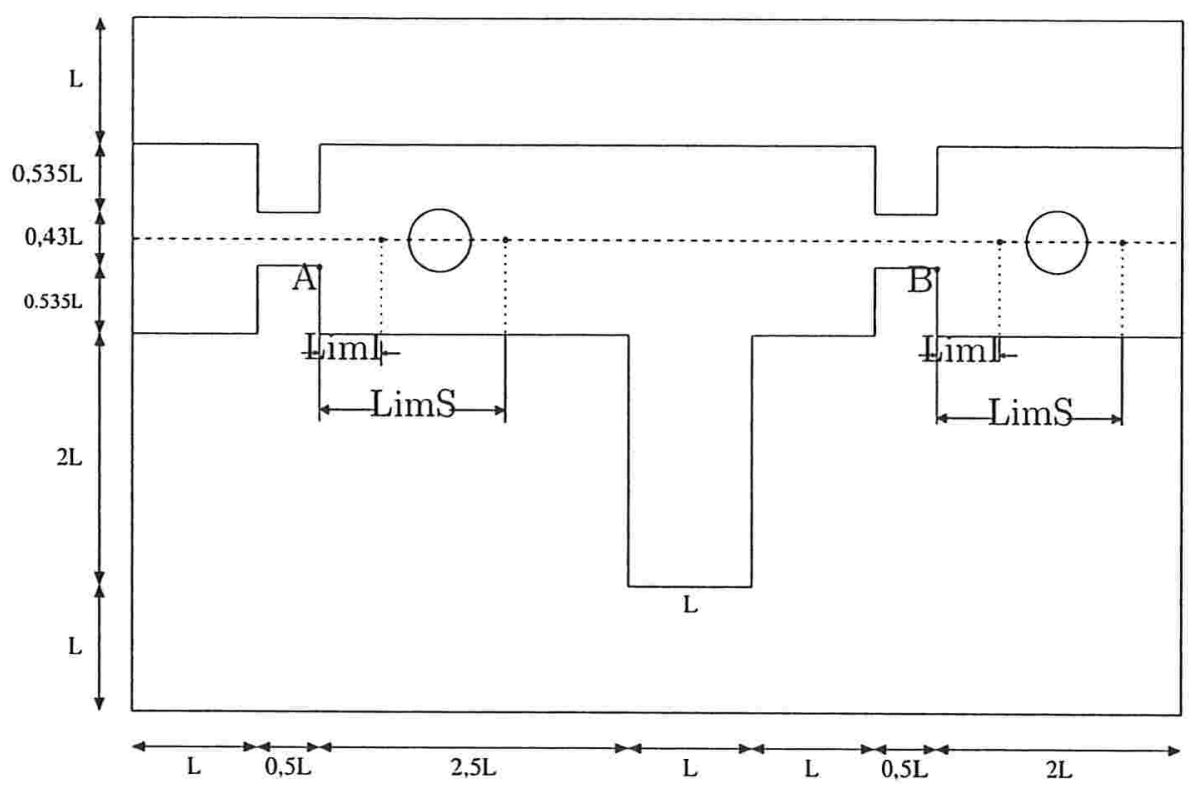

Figura 5.16: Exemplo da localização de limitantes mecânicos para o movimento do centro de massa do cilindro imerso.

superior. As distribuições temporais dos centros de massa relativas a três posições iniciais distintas do centro de massa do cilindro 1 , mantendo-se a mesma posição inicial do centro de massa do cilindro 2, são apresentadas nas Figuras 5.17 (a), (b) e (c).

No primeiro experimento (Figura 5.17 (a) ) o centro de massa do cilindro 1 se encontra em xcm1 $=0,013 \mathrm{~m}$ em relação ao ponto $\mathrm{A}$ ( uma posição à direita do limite inferior) e o centro de massa do cilindro 2 se encontra em $x \mathrm{~cm} 2=0,012 \mathrm{~m}$ em relação ao ponto B. Em $\mathrm{t}=0$ o pistão inicia o seu movimento de ascensão, aumentando a pressão no conjunto cilindro-canal. Com o aumento da pressão, o cilindro 1 se desloca para a esquerda, em direção ao limite inferior, atingindo-o em aproximadamente $t=0,018 \mathrm{~s}$. Neste instante o seu movimento para a esquerda é interrompido, pelo limitador mecânico, e ele permanece em repouso até $\mathrm{t}=0,062 \mathrm{~s}$. O pistão, neste momento, inicia seu movimento de descida, diminuindo a pressão no interior do canal e promovendo o movimento do cilindro 1 para à direita do limite inferior e em direção ao limite superior. Em $t=0,105$ s ele atinge $o$ seu deslocamento máximo, sem atingir, no entanto, o limite superior. Observa-se que seu deslocamento máximo em relação ao limite inferior, neste primeiro ciclo do pistão, é de $4,0 \times 10^{-3} \mathrm{~m}$. Fez-se, assim a análise do movimento do cilindro 1 no primeiro ciclo do pistão. Passa-se agora à análise do movimento do cilindro 2 neste mesmo ciclo. O cilindro 
2 parte da posição $\mathrm{xcm} 2=0,012 \mathrm{~m}$ em $\mathrm{t}=0$ s. Como o pistão se desloca para acima, o cilindro 2 se desloca para a direita, em direção ao limite superior (linha horizontal superior LimS). Enfatiza-se que a mesma linha horizontal, representa os dois limites superiores

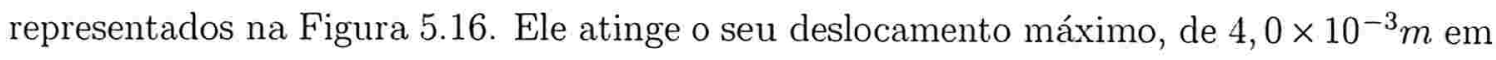
$\mathrm{t}=0,06 \mathrm{~s}$, quando retorna em direção ao limite inferior, devido à descida do pistão.

Os movimentos dos cilindros não permanecem sincronizados com o movimento do pistão devido às inércias dos mesmos. A falta de sincronia leva ao amortecimento do deslocamento do cilindro 2 , o que pode ser observado durante o terceiro ciclo de movimento.

Passando agora à análise do caso da Figura 5.17 (b), para o qual xcm1 $=0,018 \mathrm{~m}$ e $\mathrm{xcm} 2=0,012 \mathrm{~m}$ em $\mathrm{t}=0$. Observa-se que, o cilindro 1 , oscila entre os limites inferior e superior, sem no entanto, atingi-los, até $t=0,25 \mathrm{~s}$ quando ele atinge o limite inferior e permanece parado até $\mathrm{t}=0,26 \mathrm{~s}$. Novamente o cilindro 2, que parte da mesma posição do experimento (a), tem o seu movimento amortecido devido à falta de sincronia com o pistão e também devido à dinâmica do escoamento no sistema.

Na Figura 5.17 (c) mostram-se os resultados para o caso em que o cálculo foi iniciado com $\mathrm{xcm} 1=0,024 \mathrm{~m}$. Neste caso, a simulação se iniciou com o cilindro 1 tendo seu centro de massa coincidente com o seu limite superior. No seu movimento ele atinge este limite superior no final do segundo ciclo. $\mathrm{O}$ cilindro 2 tem novamente o seu movimento amortecido, porém, com uma intensidade menor que nos casos prescedentes. Isto mostra que a dinâmica de um cilindro é dependente da dinâmica do outro.

No próximo experimento, apresentado na Figura 5.18, iniciou-se o cálculo com os dois cilindros localizados na mesma posição relativa ao limite inferior de cada cilindro, ou seja $\mathrm{xcm} 1=\mathrm{xcm} 2=0,018 \mathrm{~m}$ em $\mathrm{t}=0$. Neste caso observa-se que os cilindros se movimentam com um desfazagem de meio ciclo o que é fisicamente consistente, uma vez que os movimentos dos mesmos é gerado por um mesmo pistão. No entanto observa-se que ambos vão se deslocando em direção aos respectivos limites inferiores. Em $t=0,252 \mathrm{~s}$ o cilindro 1 atinge o limite inferior e permance parado por aproximadamente 0,01 s o que afeta visivilmente a dinâmica do cilindro 2 .

O próximo caso que foi simulado corresponde à seguinte condição inicial e condição de operação: os dois cilindro foram posicionados, inicialmente, de forma a que o cilindro 1 está no limite superior e o cilindro 2 está no limite inferior. Desta forma xcm $1=0,0009 \mathrm{e}$ $\mathrm{xcm} 2=0,0032$. Os limites inferiores e os superiores para ambos cilindros foram alterados em relação aos casos precedentes, de tal forma que os cilindros poçam atingir os cantos das contrações colocadas antes e após a cavidade de fundo móvel (como ilustrada na 


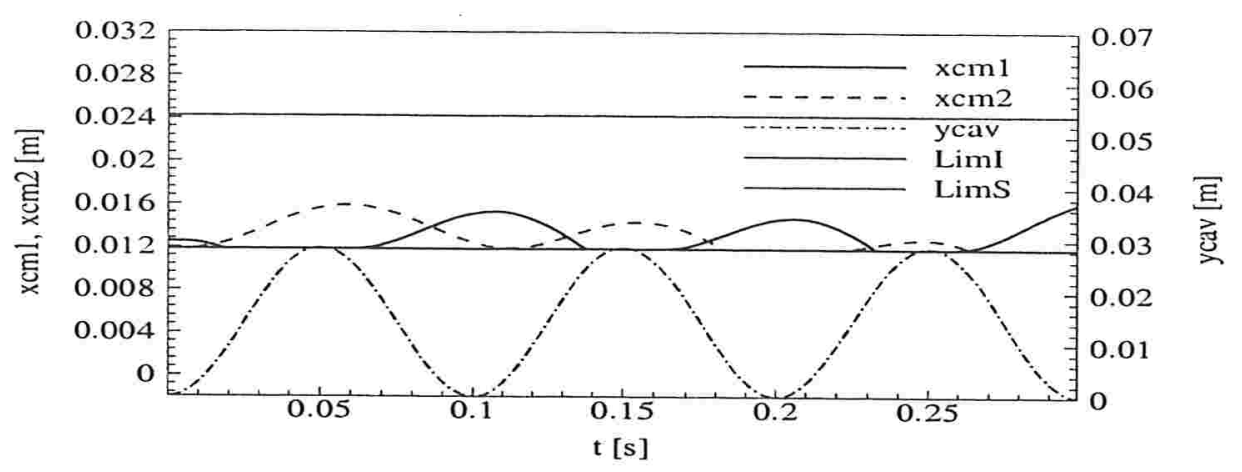

(a) $\mathbf{X}_{c m 1}=(0,013,0,005)$ e $\mathbf{X}_{c m 2}=(0,012,0,005)$

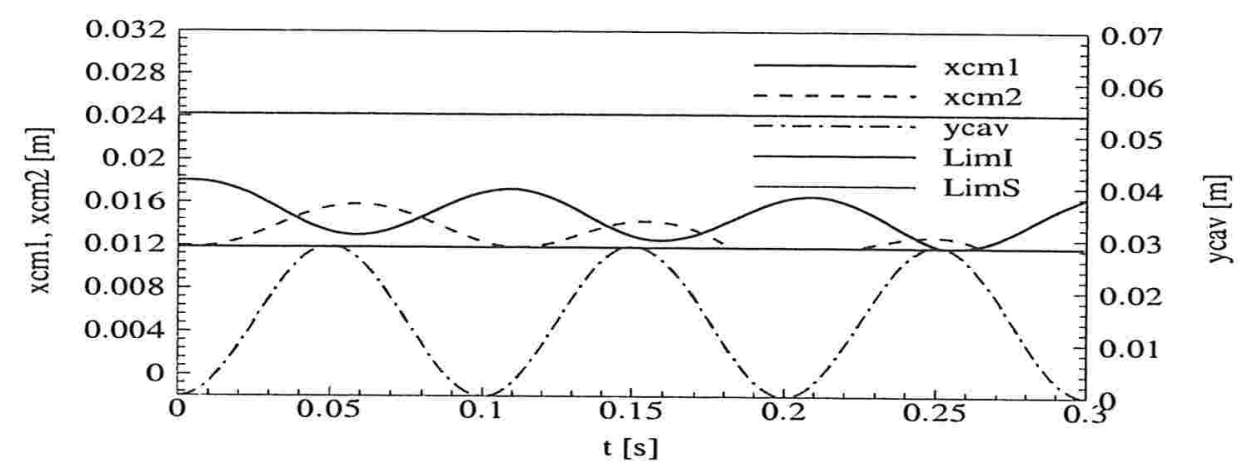

(b) $\mathbf{X}_{c m 1}=(0,018,0,005)$ e $\mathbf{X}_{c m 2}=(0,012,0,005)$

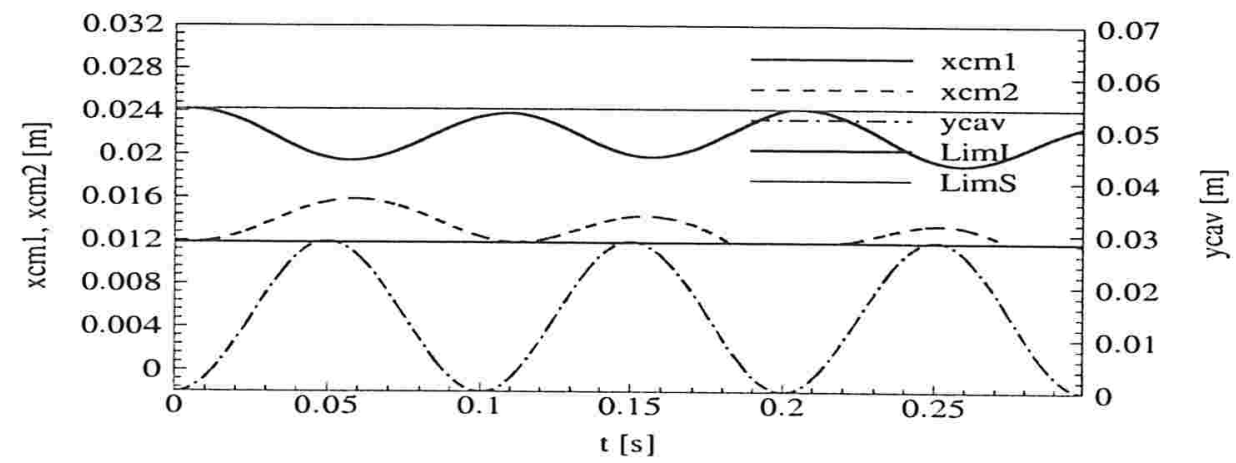

(c) $\mathbf{X}_{c m 1}=(0,024,0,005)$ e $\mathbf{X}_{c m 2}=(0,012,0,005)$

Figura 5.17: Movimento dos cilindos limitados mecanicamente: abscissas relativas do centro de massa dos cilindros ( $\mathrm{xcm} 1 \mathrm{e} \mathrm{xcm} 2)$ aos pontos A e B das contrações; altura do fundo móvel da cavidade, ycav; linhas horizontais inferior e superior, representam o valor de LimI e LimS, respectivamente $\left(\rho_{c}=1,5 \rho\right.$ e $\left.v_{0}=0,9 \mathrm{~m} / \mathrm{s}\right)$. 


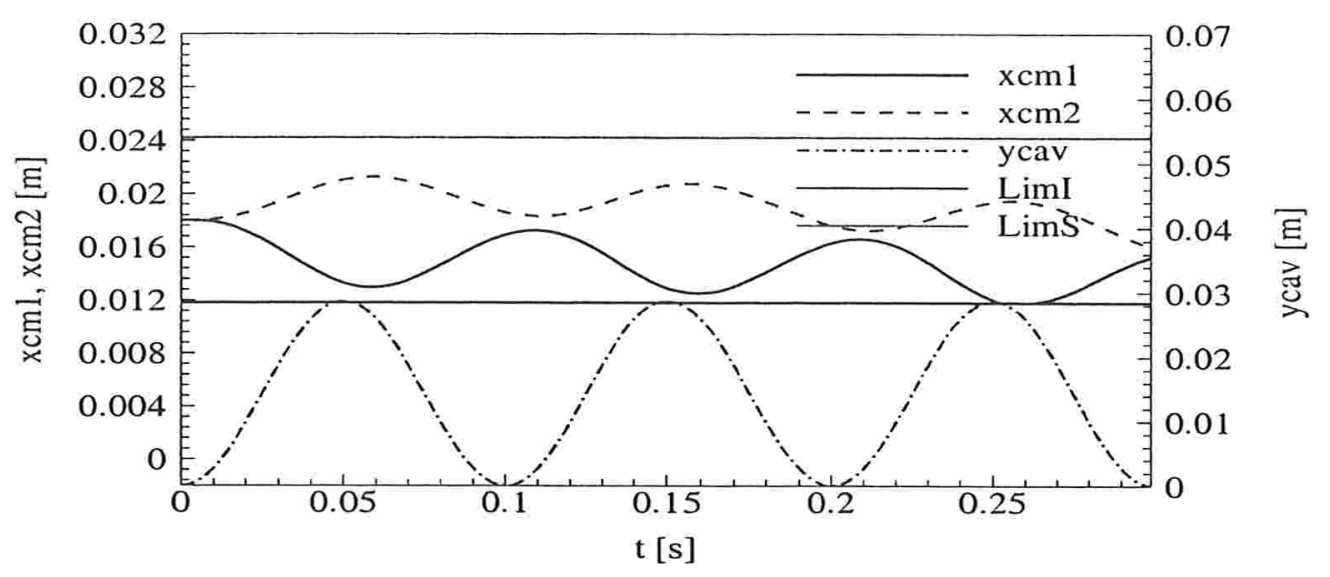

Figura 5.18: Movimento dos cilindos limitados mecanicamente: $\mathbf{X}_{c m 1}=\mathbf{X}_{c m 2}=(0,018$, $0,005)\left(\rho_{c}=1,5 \rho\right.$ e $\left.v_{0}=0,9 \mathrm{~m} / \mathrm{s}\right)$.

Figura 5.13). Nos casos precedentes isto não era permitido. Além disso, o espaçamento entre os limites foi considerado igual ao raio dos cilindros e adoto-se para a amplitude da velocidade do fundo móvel $V_{0}=1,45 \mathrm{~m} / \mathrm{s}$ (equação 5.13 ).

Observa-se na Figura 5.19 que ambos os cilindros tocam os limites inferior e superior até o tempo $\mathrm{t}=0,3 \mathrm{~s}$. Neste momento acontece uma anomalia nos movimentos de ambos os cilindros: eles deixam de tocar os limites inferiores. Dois ciclos ainda permanecem até que em $t=0,37 \mathrm{~s}$ os dois cilindros permanecem constantemente nos limites superiores e as passagens do fluido pelas restrições fica completamente abertas. Estas anomalias não podem ainda ser explicadas do ponto de vista físico. Restam explicações ligadas à modelagem matemática que pode estar apresentando problemas. Especificamente nada de especial foi feito para modelar os toques dos dois cilindros com as batentes das restrições nos canais. Maiores investigações devem ser feitas.

Na Figura 5.20 apresenta-se o resultado do movimento do centro de massa do cilindro 1 e 2 para um problema com as mesmas condições de operação que do caso anterior, mas com o dobro de espaçamento de separação entre a cavidade e a fronteira inferior do domínio. Pode-se observar que a anomalia do experimento prescedente não acontece neste maior domínio de estudo, mas um fenómeno diferente aparece. Observe-se que até $t=0,38 \mathrm{~s}$ os cilindros desenvolvem a dinâmica esperada de abertura e fechadura das válvulas. No intervalo de tempo $t=0,38 \mathrm{~s}$ até $t=0,41 \mathrm{~s}$ ambas não conseguem atingir seus respectivos limitantes. Observe-se que apartir do tempo $t=0,41 \mathrm{~s}$, o primeiro cilindro permanece fechado, enquanto que o cilindro 2 alterna entre aberto e fechado. Isto é, 
existe um intervalo de tempo onde ambas válvulas estão fechadas enquanto o pistão injeta e retira fluido. Este fenômeno nào é físico e precisa de maiores pequisas do porque este acontecimento.

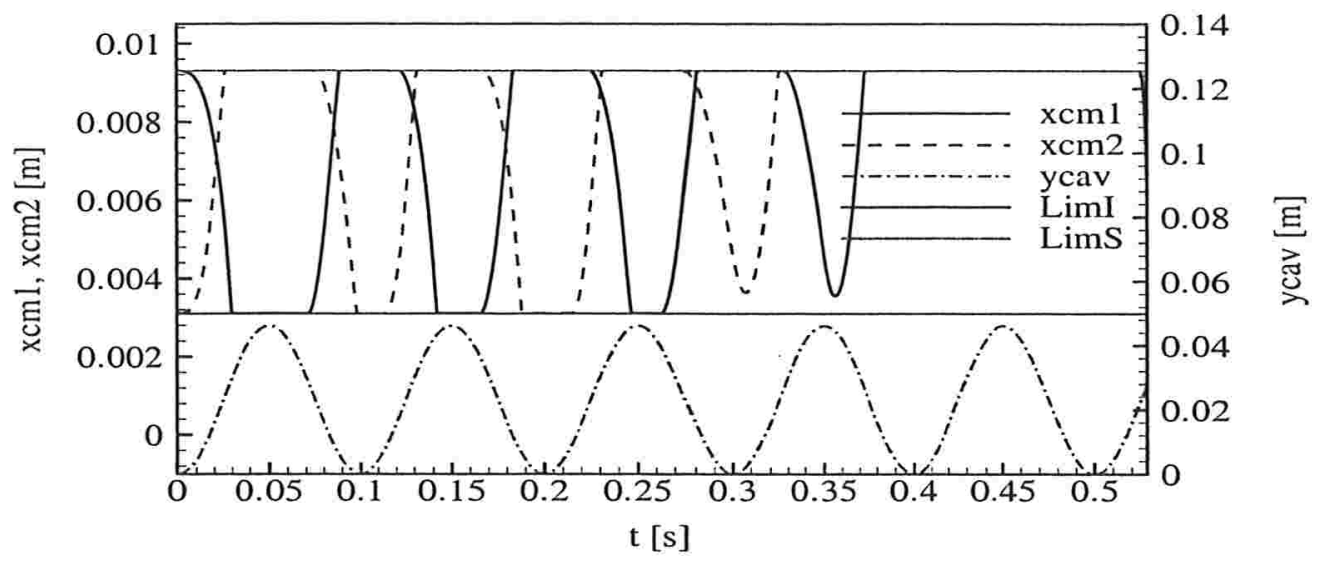

Figura 5.19: Movimento dos cilindos limitados mecanicamente: $X_{c m 1}=(0,003,0,005)[\mathrm{m}]$ e $\mathbf{X}_{c m 2}=(0,009,0,005)[\mathrm{m}]\left(\rho_{c}=1.5 \rho\right.$ e $\left.V_{0}=1.45 \mathrm{~m} / \mathrm{s}\right)$.

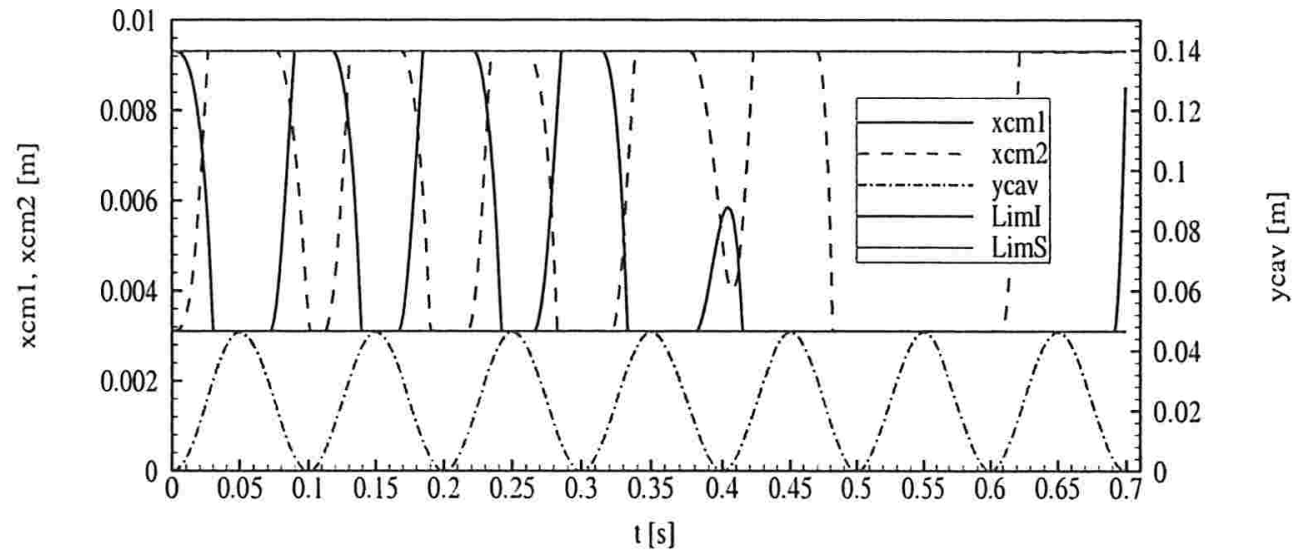

Figura 5.20: Movimento dos cilindos limitados mecanicamente: $X_{c m 1}=(0,003,0,005)[\mathrm{m}]$ e $\mathrm{X}_{c m 2}=(0,009,0,005)[\mathrm{m}]\left(\rho_{c}=1.5 \rho\right.$ e $\left.V_{0}=1.45 \mathrm{~m} / \mathrm{s}\right)$. Espaço de separação entre a cavidade e a fronteira inferior do domínio duas vezes maior ao da experiência anterior (Figura 5.19).

Para melhorar o entendimento do escoamento no sistema, mostra-se na Figura 5.21, os campos de pressão ao longo do primeiro ciclo do caso da Figura 5.18. Na Figura 5.21 (a) o pistão começa a subir, elevando a pressão no interior do sistema. As cores mostram as 
variações de pressão. Junto ao cilindro a cor vermelha indica uma região de alta pressão e à medida que se observa este campo em direção às válvulas vê-se que acontece uma queda nos seus valores o que promove o escoamento. Na Figura 5.21 (b) o campo de pressão já é mais uniforme em toda a região entre as válvulas e a cabeça do pistão. Entre as Figuras 5.21 (b) e (c) tem-se $t=0,025$ s que corresponde à máxima velocidade do pistão, e também a uma aceleração nula. O pistão está a meio caminho do seu curso. A partir deste momento o pistão começa a reduzir sua velocidade, ou seja, a se desacelerar. Em conseqüência a pressão no fluido junto ao pistão cai, descalerando o fluido no inteior do sistema. Na Figura 5.21 (d) observe-se, pelas cores que o gradiente de pressão na cavidade se inverte, quando comparada com a Figura 5.21 (b). Na Figura 5.21 (e) o cilindro atinge o seu ponto morto superior. Observa-se claramente um gradiente inverso de pressão sobre a cabeça do pistão. No interior do cilindro, acima do pistão a pressão é negativa e abaixo do pistão ela é positiva. No momento em que o pistão começa sua descida (Figura 5.21 (f)) o fluido acima do pistão começa a ser succionado para o interior do cilindro e abaixo do pistão ele começa a ser expulso. O processo prossegue até que na Figura 5.21 (j) o pistão chega ao seu ponto morto inferior.

Toda esta dinâmica do pistão afeta também o movimento das válvulas. Cabe observar que a dinâmica destas válvulas depende também do campo do escoamento sobre elas assim como de suas inércias.

Na Figura 5.22 mostra-se a norma do vetor força euleriana nas vizinhanças do canal, cavidade e fundo móvel. Observa-se que a força desenha corretamente as fronteiras que são modeladas virtualmente. Quanto maior é a pressão no interior do sistema (Figura 5.21 (b)-(c)) maior a norma da força sobre as paredes ( Figura 5.22 (b)-(c)). Observa-se ainda que na fase de descida do pistão, os momentos de menor pressão (Figura $5.21(\mathrm{~g})$-(h)) também correspondem a maior norma da força euleriana (Figura $5.22(\mathrm{~g})$-(h)).

Na Figura 5.23 mostra-se a componente vertical da força euleriana que as fronteiras virtuais "distribuem" para o fluido. Uma análise destas figuras é importante para verificar a consistência da metodologia. Tomando como exemplo a Figura 5.23 (b), que representa um momento em que o pistão está subindo, ou seja o fluido está sendo comprimido e expulso do sistema. Observa-se na vizinhança da parede superior que o fluido está sendo submetido a uma força no sentido negativo do eixo y impedindo-o de sair pela parede para o exterior do canal. Por outro lado, nas vizinhanças da parede inferior do canal, observamse valores positivos da força injetada pela fronteira virtual sobre o fluido, impedindo que surja escoamento sobre a parede, do interior para o exterior do canal. De forma bastante interessante, observa-se o inverso na Figura 5.23 (h) quando o pistão está descendo. Estes 
resultados são bastantes consistentes fisicamente.

Na Figura 5.24 mostra-se a componente horizontal da velocidade ilustrando fatos físicamente interessantes e consistentes. Por exemplo, na Figura 5.24 (b), quando o pistão está subindo observa-se o movimento do fluido passando entre os dois estrangulamentos onde esta componente de velocidade atinge valores máximos. No estrangulamento à direita do pistão a velocidade é positiva. Á sua esquerda ela é negativa. É interessante observar que o fluido está também acompanhando o pistão pelo lado externo sendo succionado para o seu interior. Olhando a Figura 5.24 (h) observa-se uma inversão completa de todo o movimento, uma vez que o pistão está descendo.

A Figura 5.25, que apresenta a componente vertical da velocidade, corrobora a análise prescedente. 

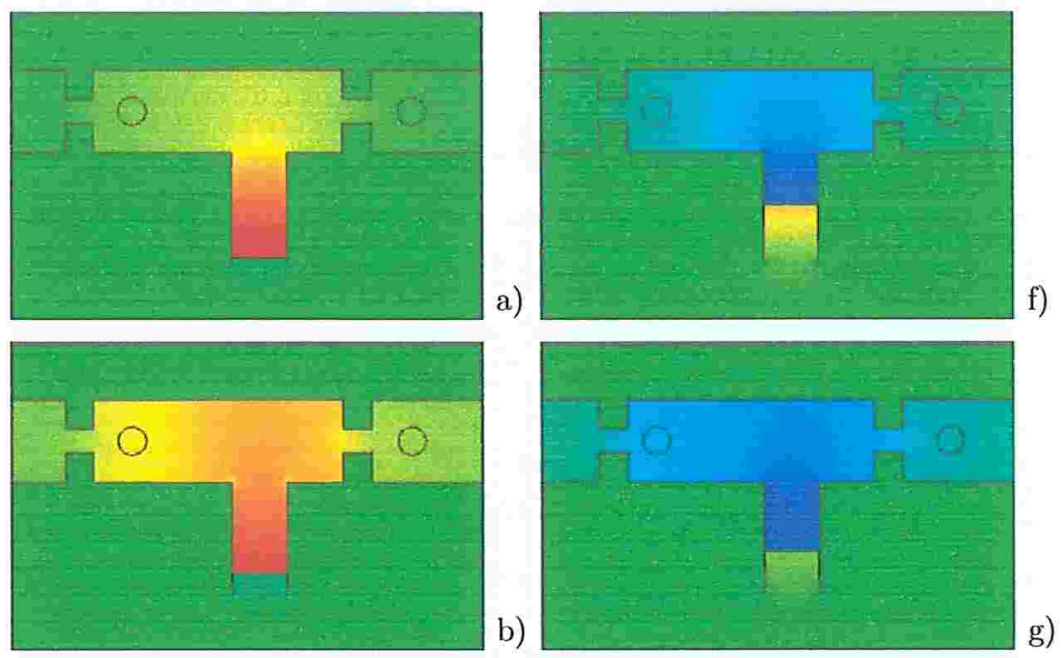

g)
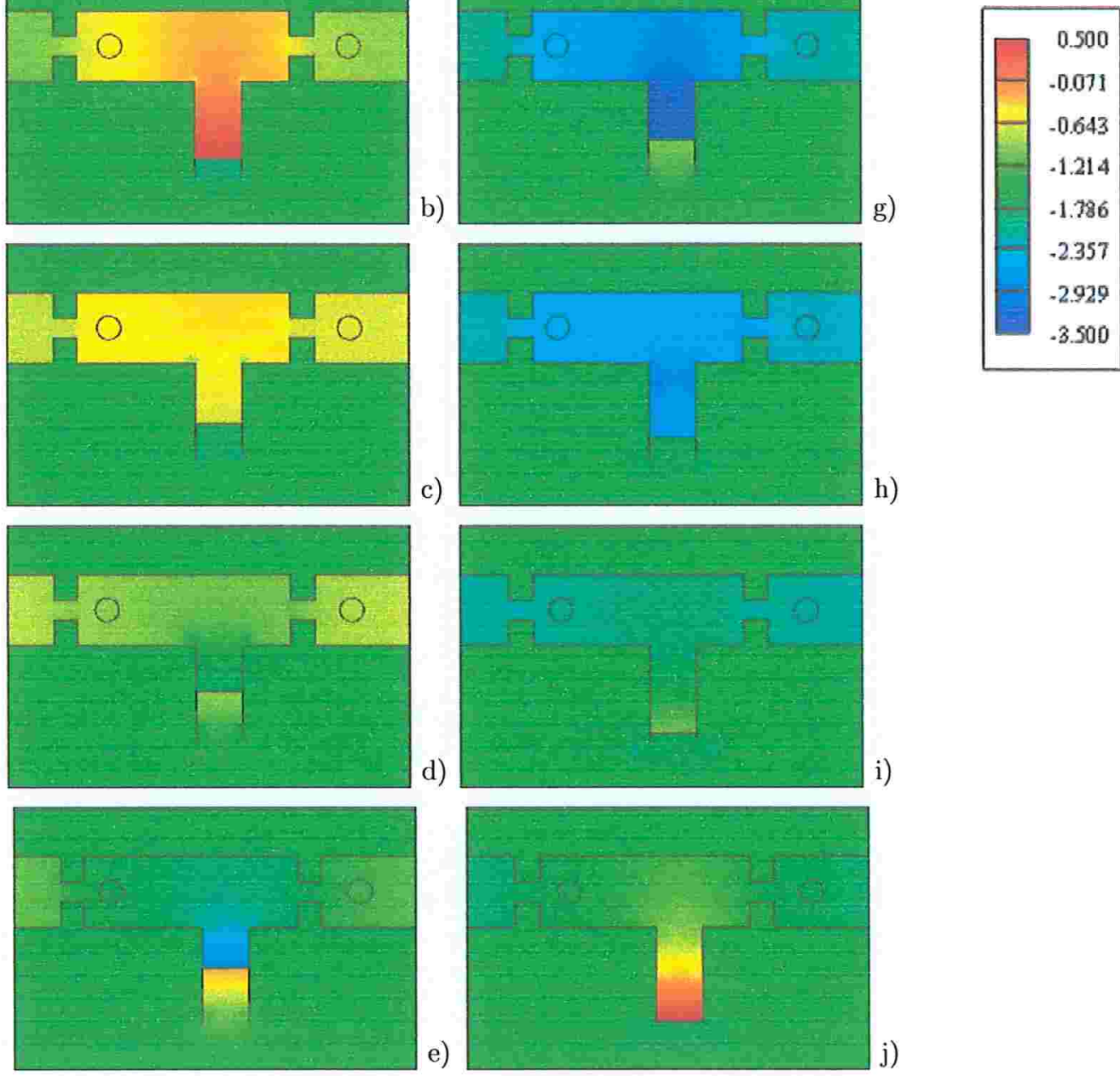

Figura 5.21: Escoamento no interior de um conjunto canal-cavidade com fundo móvel contendo dois cilindros imersos. Campos de pressão para o intervalo de tempo $0<t \leq 0,1$, $\Delta t=0,01 s$. 

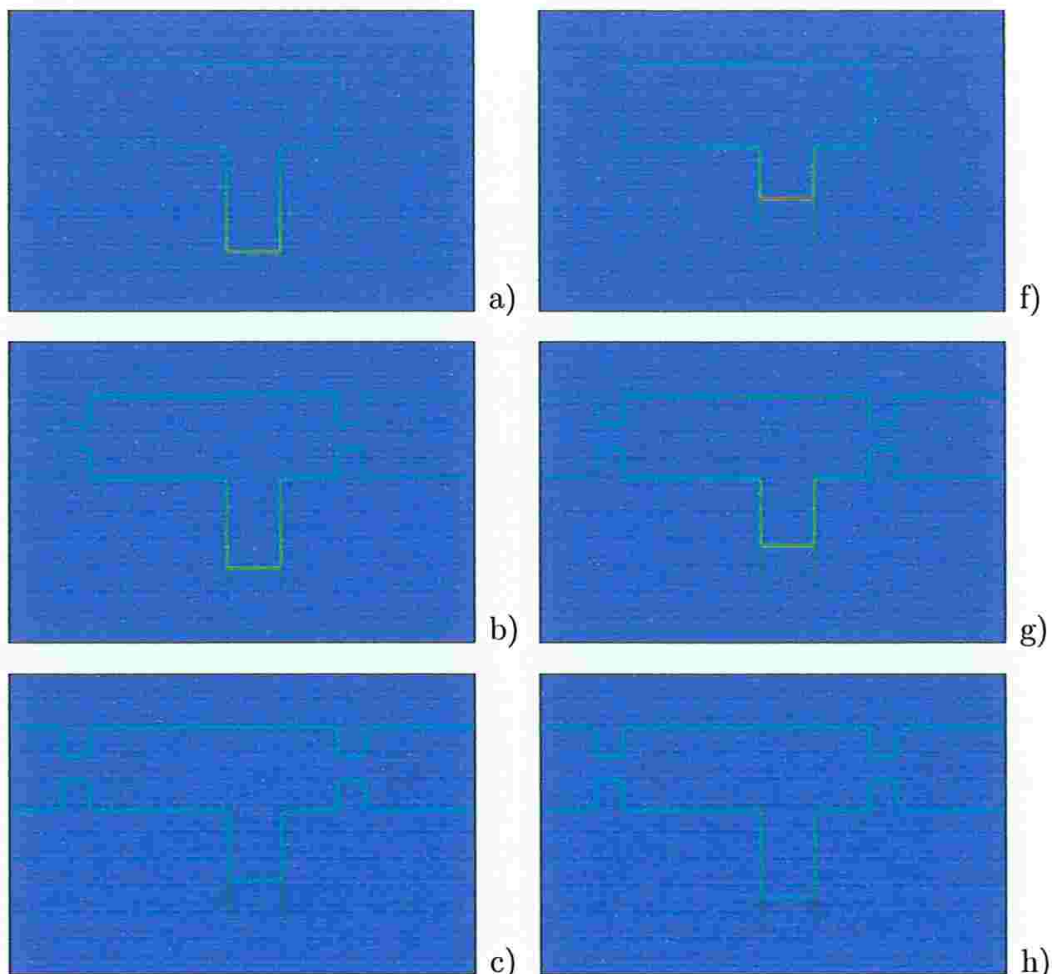

g)

\begin{tabular}{|l|}
2268 \\
1998 \\
1728 \\
1458 \\
1188 \\
918 \\
648 \\
378 \\
\hline
\end{tabular}
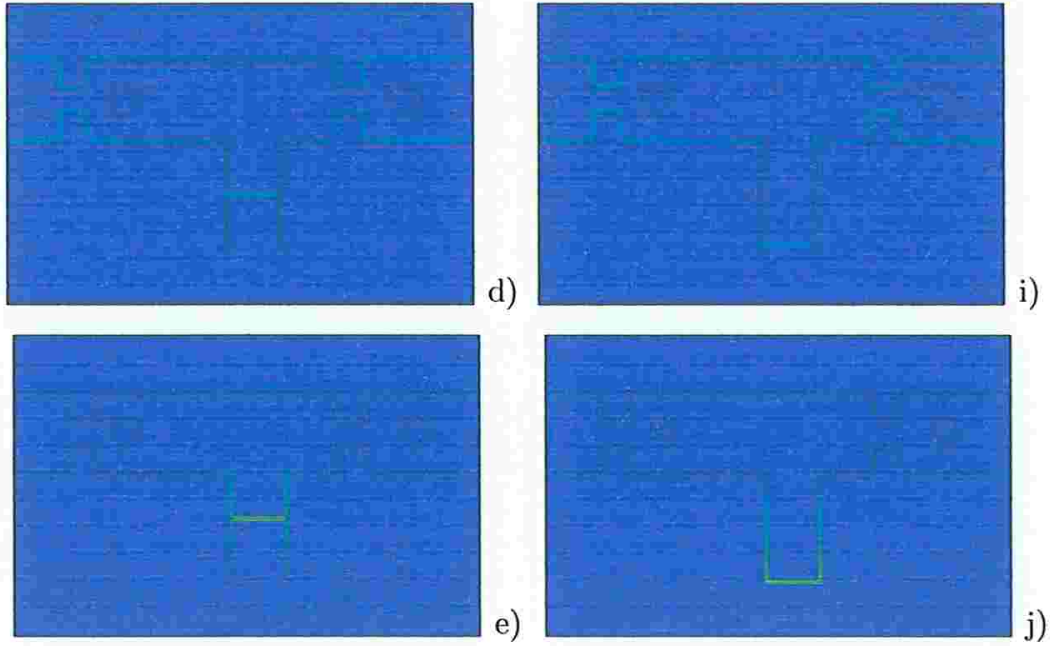

h)

Figura 5.22: Campos da norma da força euleriana para o intervalo de tempo $0<t \leq 0.1$, $\Delta t=0,01 s$. 

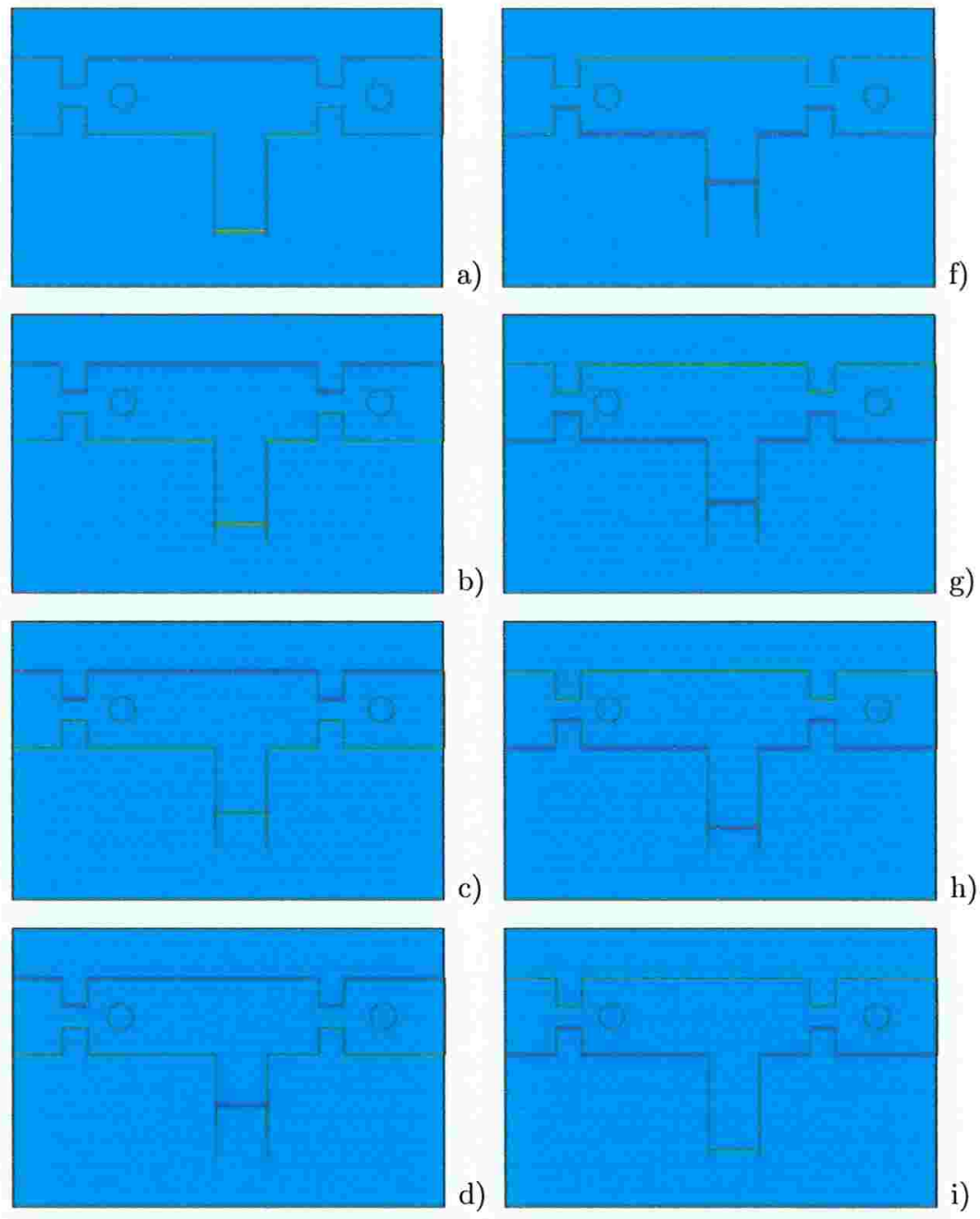

$$
\text { h) }
$$
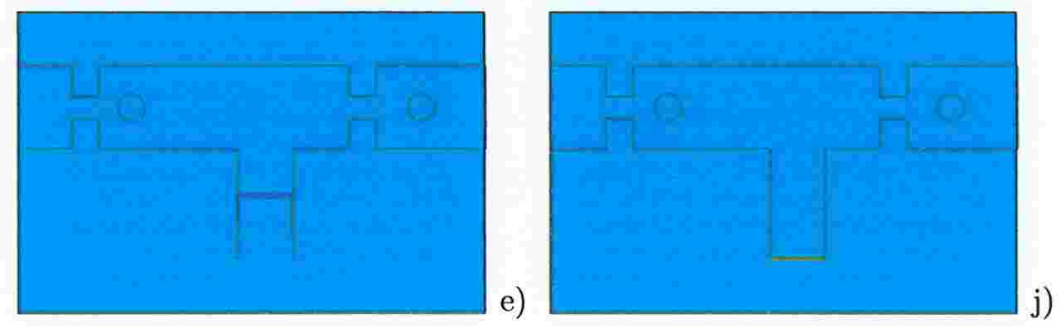

Figura 5.23: Campos da força euleriana: componente vertical durante o intervalo de tempo $0<t \leq 0,1, \Delta t=0,01 s$. 

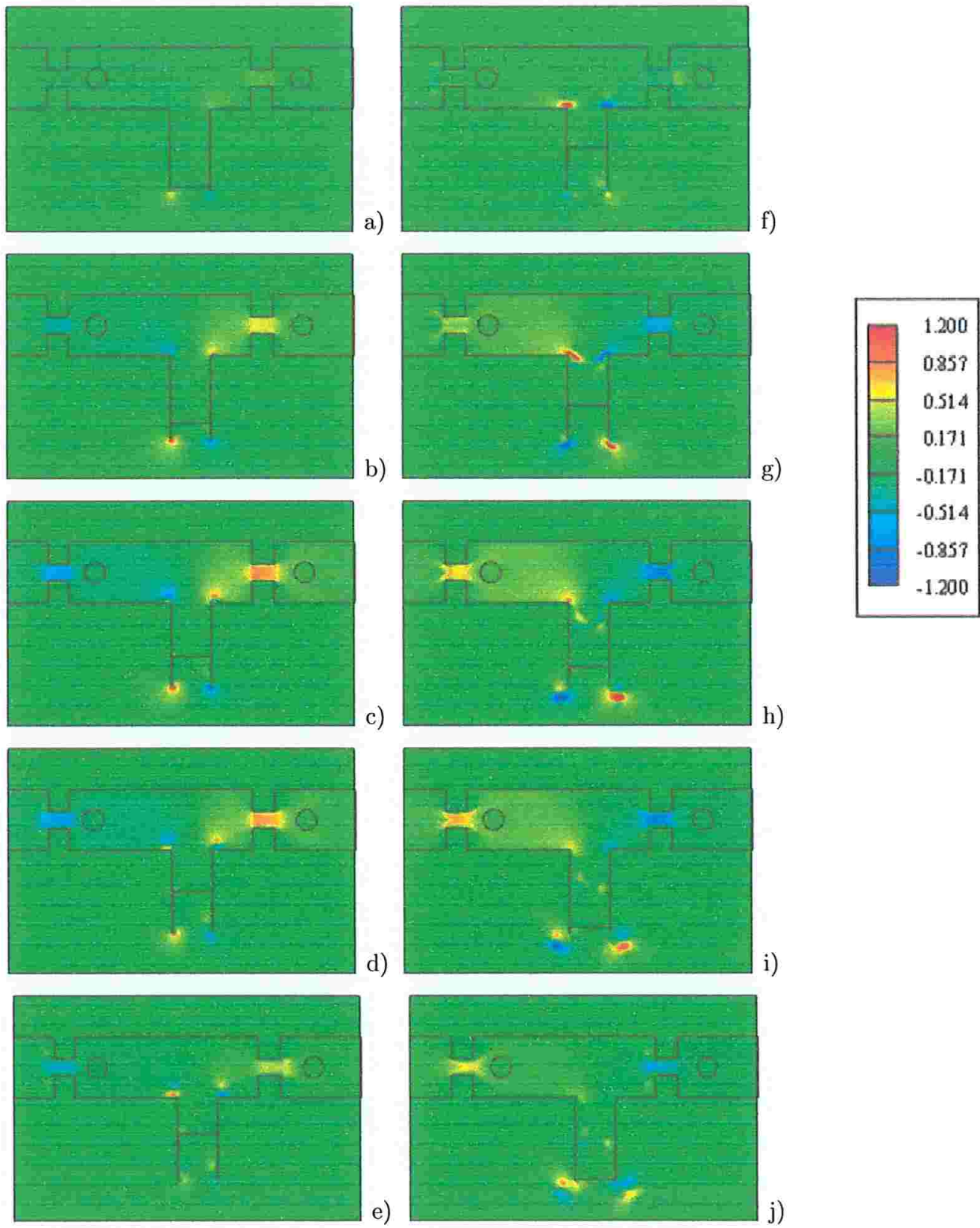

Figura 5.24: Escoamento no interior de um conjunto canal-cavidade com fundo móvel contendo dois cilindros imersos. Campos de velocidade: componente horizontal para o intervalo de tempo $0<t \leq 0.1, \Delta t=0,01 s$. 

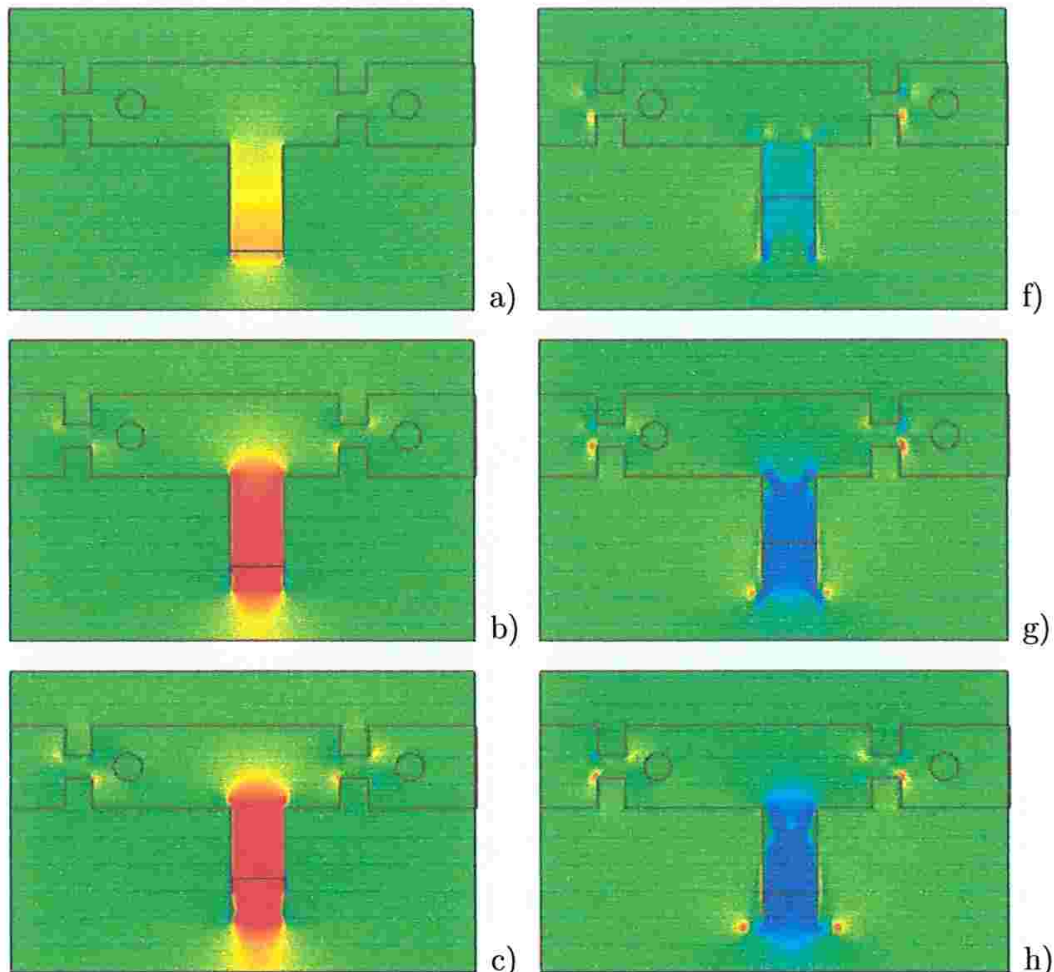

g)
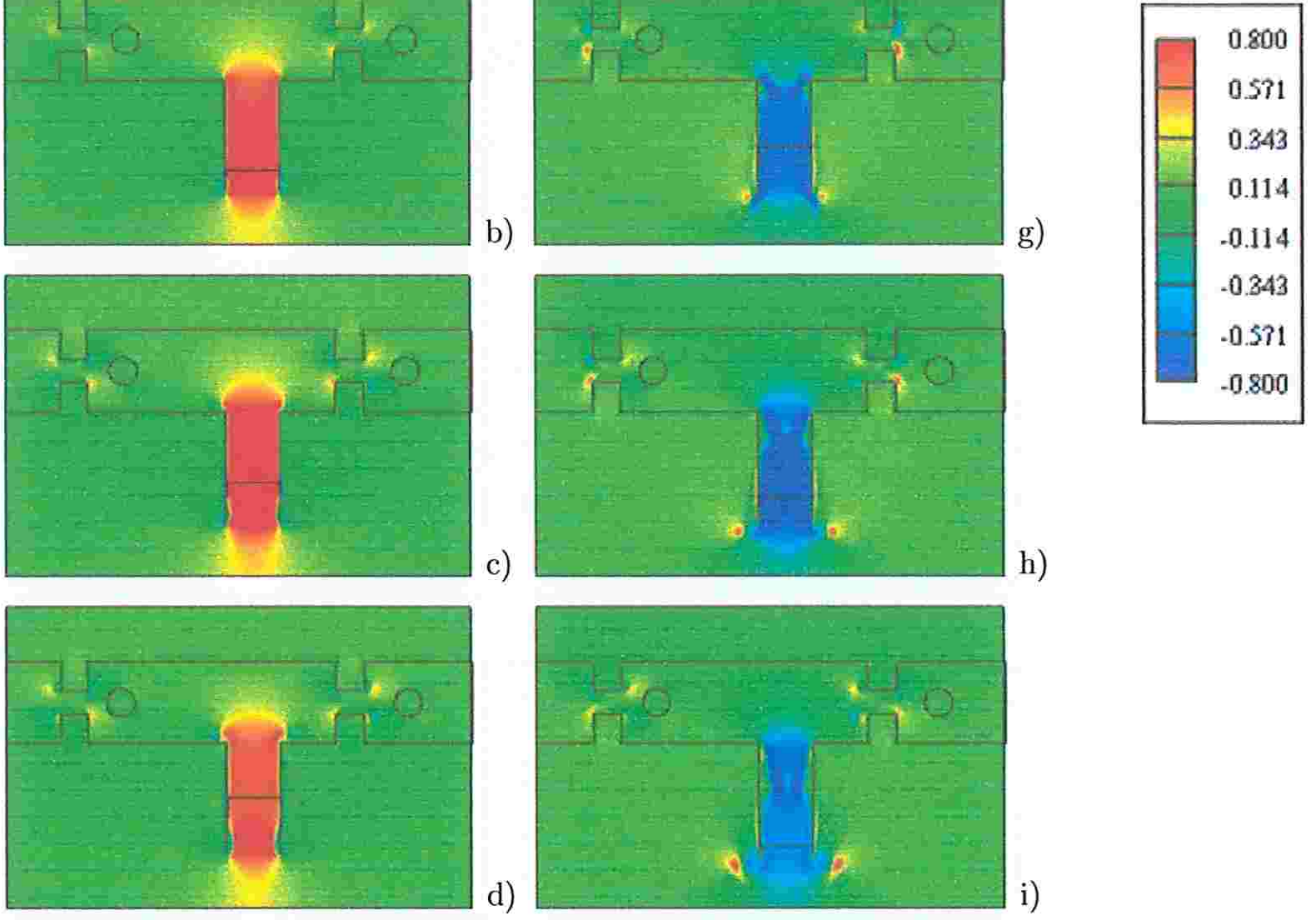

h)
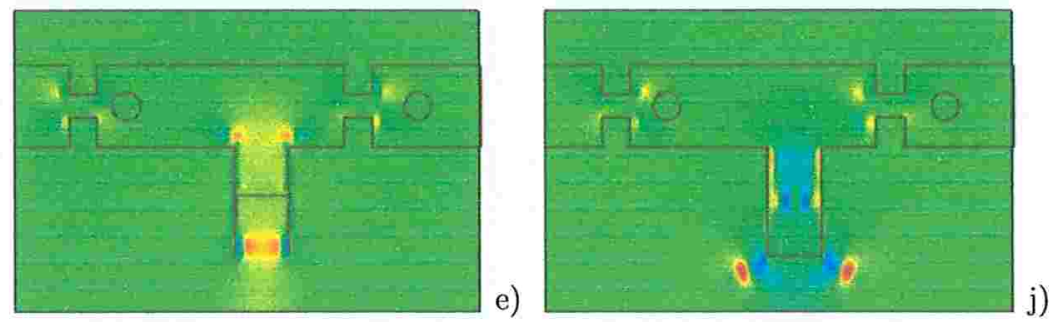

Figura 5.25: Escoamento no interior de um conjunto canal-cavidade com fundo móvel contendo dois cilindros imersos. Campos da velocidade: componente vertical para o intervalo de tempo $0<t \leq 0,1, \Delta t=0,01$ s. 


\section{Conclusão}

Muitos escoamentos presentes em problemas práticos são caracterizados por geometrias complexas, móveis e mesmos deformáveis. Corações e válvulas mecânicas, motores de combustão interna, compressores e turbinas são exemplos. As metodologias de análise teórica oferecem muitos recursos para esta classe de problemas. Entre elas, destacam-se aquelas baseadas em malhas não estruturadas, utilizadas para discretizar as equações que compõem os modelos matemáticos. Uma metodologia alternativa e promisora vem sendo desenvolvida pela comunidade mundial, na busca de se poder resolver e analizar esta classe de problemas. Trata-se da metodologia de fronteira imersa.

No presente trabalho foi dada uma contribuição no desenvolvimento e aplicações desta metodologia em problemas que envolvem sistemas de bombeamento. Foi ultilizado o Modelo Físico Virtual, desenvolvido recentemente por Lima e Silva et al. [25]. Um programa computacional foi adaptado a uma primeira aproximação de um sistema de bombeamento, composto de cavidades, pistão móvel e válvulas.

O código foi validado para problemas de fronteira móvel simulando um escoamento de Couette circular, onde os cilindros foram modelados por meio de campos de forças. Uma outra validação qualitativa foi feito por intermédio da simulação de um pêndulo cilindrico bidimensional em um meio fluido parado. Uma vez validado o programa, ele foi aplicado para uma análise preliminar do escoamento gerado pelo sistema de bombeamento comentado acima.

Foi feito um estudo de influência do posicionamento inicial das válvulas. Resultados fisicamente consistentes foram obtidos. Este trabalho ainda não é conclusivo e maiores desenvolvimentos são necessários. No entanto ele serve de base para desenvolvimentos futuros, uma vez que a metodologia utilizada se mostra muito promissora. 


\section{Perspectivas}

Com os desenvolvimentos dos trabalhos de Arruda [1] e do presente trabalho, pode-se, com realismo, estabelecer as seguintes perspectivas:

- Continuação do desenvolvimento do programa e validação frente a dados experimentais, para aplicação em sistemas de bombeamento, compressores e motores de combustão interna;

- Tridimensionalização da metodologia para os casos do item anterior;

- Desenvolvimento de modelagem apropriada para o processo de colisão entre fronteiras sólidas;

- Paralelizar o código computacional para viabilizar simulações tridimensionais;

- Estender o desenvolvimento da metodologia de fronteira imersa, buscando aumentar a ordem de precisão da mesma. 
APÊNDICE A

\section{Discretização no espaço}

Aproximações de diferenças finitas de segunda ordem centradas são usadas para as derivadas espaciais dos termos das equações de Navier-Stokes. No caso da discretização das condições de contorno, aproximações centradas e não centradas de segunda ordem serão usadas de acordo com o caso.

A seguinte notação é usada: $\operatorname{conv}(\mathbf{u})$ representa o termo convectivo das equações de Navier-Stokes; difu(u) representa o termo difusivo ou dissipativo das equações de NavierStokes; $O\left(\Delta x^{2}\right)$ representa grandezas da ordem $\left(\Delta x^{2}\right)$.

\section{A.1 Discretização dos termos advectivos}

O segundo termo da equação do movimento (termo advectivo), pode ser representado, para as componentes $x$ e $y$, como mostrado na equação (A.1) e (A.2), respectivamente:

$$
\begin{aligned}
& \operatorname{conv}(\mathbf{u})_{x}=\frac{\partial(u u)}{\partial x}+\frac{\partial(v u)}{\partial y} \\
& \operatorname{conv}(\mathbf{u})_{y}=\frac{\partial(u v)}{\partial x}+\frac{\partial(v v)}{\partial y} .
\end{aligned}
$$

As equações (A.1) e (A.2) são discretizadas, respectivamente, conforme as equações a seguir, utilizando-se o esquema centrado de segunda ordem. 


$$
\begin{aligned}
& \operatorname{conv}(\mathbf{u})_{x}=\left\{\begin{array}{l}
\frac{1}{\Delta x}\left[\left(\frac{u_{i, j+1}+u_{i, j}}{2} \cdot \frac{u_{i, j+1}+u_{i, j}}{2}\right)-\left(\frac{u_{i, j}+u_{i, j-1}}{2} \cdot \frac{u_{i, j}+u_{i, j-1}}{2}\right)\right] \\
+ \\
\frac{1}{\Delta y}\left[\left(\frac{u_{i+1, j}+u_{i, j}}{2} \cdot \frac{v_{i+1, j}+v_{i+1, j-1}}{2}\right)-\left(\frac{u_{i, j}+u_{i-1, j}}{2} \cdot \frac{v_{i, j}+v_{i, j-1}}{2}\right)\right]
\end{array}\right\} \\
& \operatorname{conv}(\mathbf{u})_{y}=\left\{\begin{array}{l}
\frac{1}{\Delta x}\left[\left(\frac{u_{i, j+1}+u_{i-1, j+1}}{2} \cdot \frac{v_{i, j+1}+v_{i, j}}{2}\right)-\left(\frac{u_{i, j}+u_{i-1, j}}{2} \cdot \frac{v_{i, j-1}+v_{i, j}}{2}\right)\right] \\
+ \\
\frac{1}{\Delta y}\left[\left(\frac{v_{i+1, j}+v_{i, j}}{2} \cdot \frac{v_{i+1, j}+v_{i, j}}{2}\right)-\left(\frac{v_{i, j}+v_{i-1, j}}{2} \cdot \frac{v_{i, j}+v_{i-1, j}}{2}\right)\right]
\end{array}\right\}
\end{aligned}
$$

\section{A.2 Discretização do gradiente de pressão}

O gradiente de pressão nas direções $x$ e $y$, é discretizado conforme as equações abaixo

$$
\begin{aligned}
& \frac{\partial p}{\partial x}=\frac{p_{i, j}-p_{i, j-1}}{\Delta x} \\
& \frac{\partial p}{\partial y}=\frac{p_{i, j}-p_{i-1, j}}{\Delta y}
\end{aligned}
$$

\section{A.3 Discretização dos termos difusivos}

O quarto termo da equação do movimento (termo difusivo), pode ser expandido para as componentes $x$ e $y$, como mostrado nas equações (A.7) e (A.8), respectivamente

$$
\begin{gathered}
\operatorname{difu}(\mathbf{u})_{x}=\frac{\partial}{\partial x}\left[2 \nu_{e f} \frac{\partial u}{\partial x}\right]+\frac{\partial}{\partial y}\left[\nu_{e f}\left(\frac{\partial u}{\partial y}+\frac{\partial v}{\partial x}\right)\right] \\
\operatorname{difu}(\mathbf{u})_{y}=\frac{\partial}{\partial x}\left[\nu_{e f}\left(\frac{\partial v}{\partial x}+\frac{\partial u}{\partial y}\right)\right]+\frac{\partial}{\partial y}\left[2 \nu_{e f} \frac{\partial v}{\partial y}\right]+
\end{gathered}
$$

Estas componentes, $x$ e $y$, são discretizadas, respectivamente conforme Equações (A.9) e (A.12) a seguir, utilizando também o esquema centrado de segunda ordem:

$$
\frac{1}{\Delta x}\left(2 \nu_{e f, i, j} \frac{u_{i, j+1}-u_{i, j}}{\Delta x}-2 \nu_{e f, i, j-1} \frac{u_{i, j}-u_{i, j-1}}{\Delta x}\right)+\frac{1}{\Delta y}\left\{\begin{array}{l}
\nu_{e f . N}\left(\frac{u_{i+1, j}-u_{i, j}}{\Delta y}+\frac{v_{i+1, j}-v_{i+1, j-1}}{\Delta x}\right) \\
-\nu_{e f, S}\left(\frac{u_{i, j}-u_{i-1, j}}{\Delta y}+\frac{v_{i, j}-v_{i, j-1}}{\Delta x}\right)
\end{array}\right\},
$$


onde

$$
\begin{aligned}
& \nu_{e f, N}=0,25\left(\nu_{e f, i, j}+\nu_{e f, i+1, j}+\nu_{e f, i+1, j-1}+\nu_{e f, i, j-1}\right) \\
& \nu_{e f, S}=0,25\left(\nu_{e f, i, j}+\nu_{e f, i, j-1}+\nu_{e f, i-1, j-1}+\nu_{e f, i-1, j}\right)
\end{aligned}
$$

$\frac{1}{\Delta x}\left\{\begin{array}{l}\nu_{e f, E}\left(\frac{v_{i, j+1}-v_{i, j}}{\Delta x}+\frac{u_{i, j+1}-u_{i-1, j+1}}{\Delta y}\right) \\ -\nu_{e f, W}\left(\frac{v_{i, j}-v_{i, j-1}}{\Delta x}+\frac{u_{i, j}-u_{i-1, j}}{\Delta y}\right)\end{array}\right\}+\frac{1}{\Delta y}\left(2 \nu_{e f, i, j} \frac{v_{i+1, j}-v_{i, j}}{\Delta y}-2 \nu_{e f, i-1, j} \frac{u_{i, j}-v_{i-1, j}}{\Delta y}\right)$,

onde

$$
\begin{aligned}
\nu_{e f, E} & =0,25\left(\nu_{e f, i, j}+\nu_{e f, i, j+1}+\nu_{e f, i-1, j}+\nu_{e f, i-1, j+1}\right) \\
\nu_{e f, W} & =\nu_{e f, E}
\end{aligned}
$$

A discretização da equação que representa a viscosidade turbulenta é dada por:

$$
\nu_{t i, j}=\left(C_{s} l\right)^{2} \sqrt{2\left(\frac{u_{i, j+1}-u_{i, j}}{\Delta x}\right)^{2}+\left[\left(\frac{\bar{u}_{m, n}-\bar{u}_{m, s}}{\Delta y}\right)+\left(\frac{\bar{u}_{m, e}-\bar{u}_{m, w}}{\Delta x}\right)\right]^{2}+2\left(\frac{v_{i+1, j}-v_{i, j}}{\Delta y}\right)^{2}},
$$

onde

$$
\begin{aligned}
\bar{u}_{m, n} & =0,25\left(u_{i+1, j}+u_{i+1, j+1}+u_{i, j}+u_{i, j+1}\right) \\
\bar{u}_{m, s} & =0,25\left(u_{i, j}+u_{i, j+1}+u_{i-1, j}+u_{i-1, j+1}\right) \\
\bar{u}_{m, e} & =0,25\left(v_{i+1, j}+v_{i+1, j+1}+v_{i, j}+v_{i, j+1}\right) \\
\bar{u}_{m, w} & =0,25\left(v_{i+1, j-1}+v_{i+1, j}+v_{i, j-1}+v_{i, j+1}\right)
\end{aligned}
$$




\section{Condições de contornos numéricas para o passo de projeção}

B.1 Método de projeção explícito de segunda ordem

Neste trabalho o método esplícito é baseado no método de Runge-Kutta de segunda ordem (RKE2) e, este é apresentado a seguir:

Estágio 1

$$
\begin{gathered}
\rho\left(\frac{\mathbf{u}_{1}^{*}-\mathbf{u}^{\mathbf{n}}}{\Delta t / 2 .}+(\mathbf{u} \cdot \nabla \mathbf{u})^{n}\right)=-\nabla q_{1}+\nabla \cdot[2 \mu \mathbf{d}]^{n}+\mathbf{f}^{n}, \\
\mathbf{C C}\left(\mathbf{u}_{1}^{*}\right)=\mathbf{h}_{\mathbf{u}_{1}^{*},} \\
\mathbf{u}_{1}^{*}=\mathbf{u}^{n+1 / 2}+\frac{\Delta t / 2}{\rho} \nabla \phi_{1}, \\
\nabla \cdot \mathbf{u}^{n+1 / 2}=0 .
\end{gathered}
$$

Estágio 2

$$
\begin{aligned}
\rho\left(\frac{\mathbf{u}_{2}^{*}-\mathbf{u}^{\mathrm{n}}}{\Delta t}+(\mathbf{u} \cdot \nabla \mathbf{u})^{n+1 / 2}\right) & =-\nabla q_{2}+\nabla \cdot[2 \mu \mathbf{d}]^{n+1 / 2}+\mathbf{f}^{n+1 / 2}, \\
\mathbf{C C}\left(\mathbf{u}_{2}^{*}\right) & =\mathbf{h}_{\mathbf{u}_{2}^{*},} \\
\mathbf{u}_{2}^{*} & =\mathbf{u}^{n+1}+\frac{\Delta t}{\rho} \nabla \phi_{2}, \\
\nabla \cdot \mathbf{u}^{n+1} & =0 .
\end{aligned}
$$


onde: $q_{1}, q_{2}$ são aproximações para a pressão em $t^{n}$ e $t^{n+1 / 2}$, respectivamente; $\mathbf{u}_{1}^{*}, \mathbf{u}_{2}^{*}$ são as velocidades auxiliares e $\phi_{1}$ e $\phi_{2}$ os campos escalares a serem determinados. A expressão $\mathbf{C C}\left(\mathbf{u}_{l}^{*}\right)=\mathbf{h}_{\mathbf{u}_{i}^{*}}(l=1,2)$ é a respectiva condição de contorno para a velocidade auxiliar.

\section{B.1.1 Considerações adotadas na aplicação do RKE2}

A seguir apresentam-se alguma considerações usadas neste trabalho:

\section{(C1) Denominação dos pontos discretos usados}

Entende-se por ponto interno os pontos do domínio que pertencem ao interior de $\Omega$; por pontos fronteira aos pontos do domínio que pertencem à fronteira, $\partial \Omega$ e por pontos fantasmas aqueles pontos que não pertencem ao fecho de $\Omega$. Dependendo em que ponto esta localizada uma variável esta será denominada de variável interna, fronteira ou fantasma. Na Figura B.1, apresenta-se um exemplo de algumas variáveis internas, fronteiras e fantasmas.

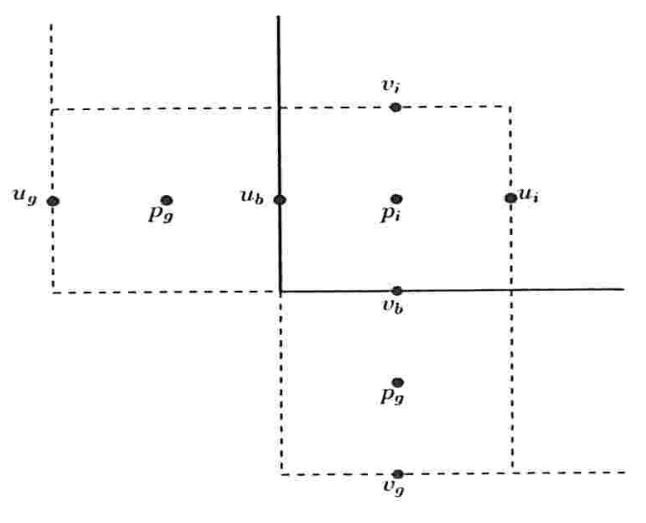

Figura B.1: $u_{i}, v_{i}, p_{i}$ : variáveis internas; $u_{b}, v_{b}$ : variáveis fronteira; $u_{g}, v_{g}, p_{g}$ : variáveis fantasmas.

(C2) Cálculo da velocidade auxiliar

Neste trabalho considerou-se que a velocidade auxiliar é calculada da seguinte forma: 
- nos pontos internos, calcula-se por intermédio da equação da quantidade de movimento dada para o escoamento

- nos pontos fronteira e fantasmas calcula-se por intermédio do uso da respectiva condição de contorno da velocidade do fluido

(C3) Relação entre os campos $p$ e $q$

Supõe-se que:

$$
\begin{array}{ll}
\frac{\partial q_{1}}{\partial x}=\frac{\partial p}{\partial x}+O\left(\Delta x^{2}\right) & , \frac{\partial q_{1}}{\partial y}=\frac{\partial p}{\partial y}+O\left(\Delta y^{2}\right) \\
\frac{\partial q_{2}}{\partial x}=\frac{\partial p}{\partial x}+O\left(\Delta x^{2}\right) & , \quad \frac{\partial q_{2}}{\partial y}=\frac{\partial p}{\partial y}+O\left(\Delta y^{2}\right)
\end{array}
$$

(C4) Tamanhos de passo no tempo e no espaço e, indezação usada

Considera-se para o passo no espaço e no tempo: $\Delta x=\alpha_{1} \Delta y$ e $\Delta t=\alpha_{2} O\left(\Delta x^{2}\right)$ (devido à condição de estabilidade no método RKE2) e, a indezação usada na discretização é mostrada na Figura B.2.

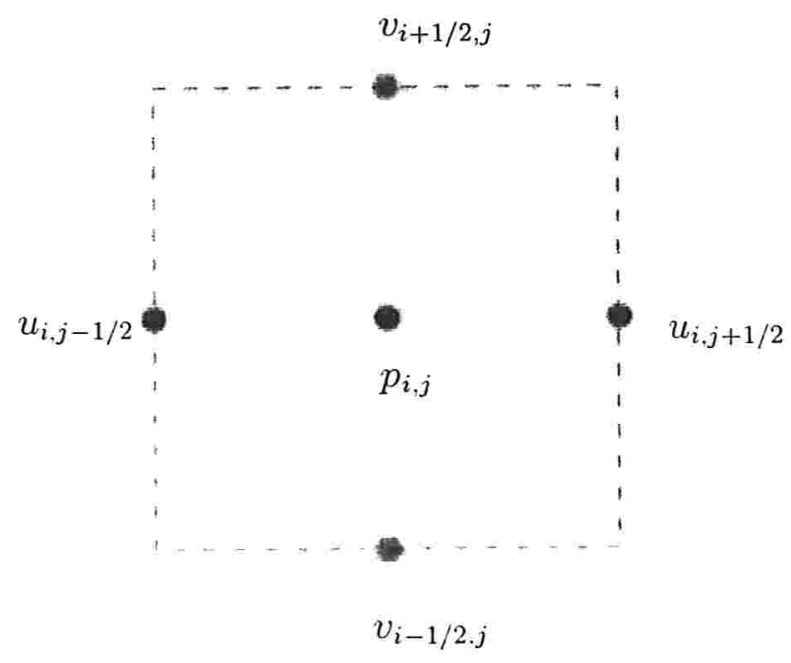

Figura B.2: Indezação usada na discretização. 
(C5) Aplicação da condição de contorno

Na Figura B.3, ilustram-se os pontos onde são aplicadas as condições de contorno do tipo Neumann para as velocidades. Os indices $(i, j)$ correspondem as células de fronteira, isto células internas com um lado pertencente à fronteira do domínio.
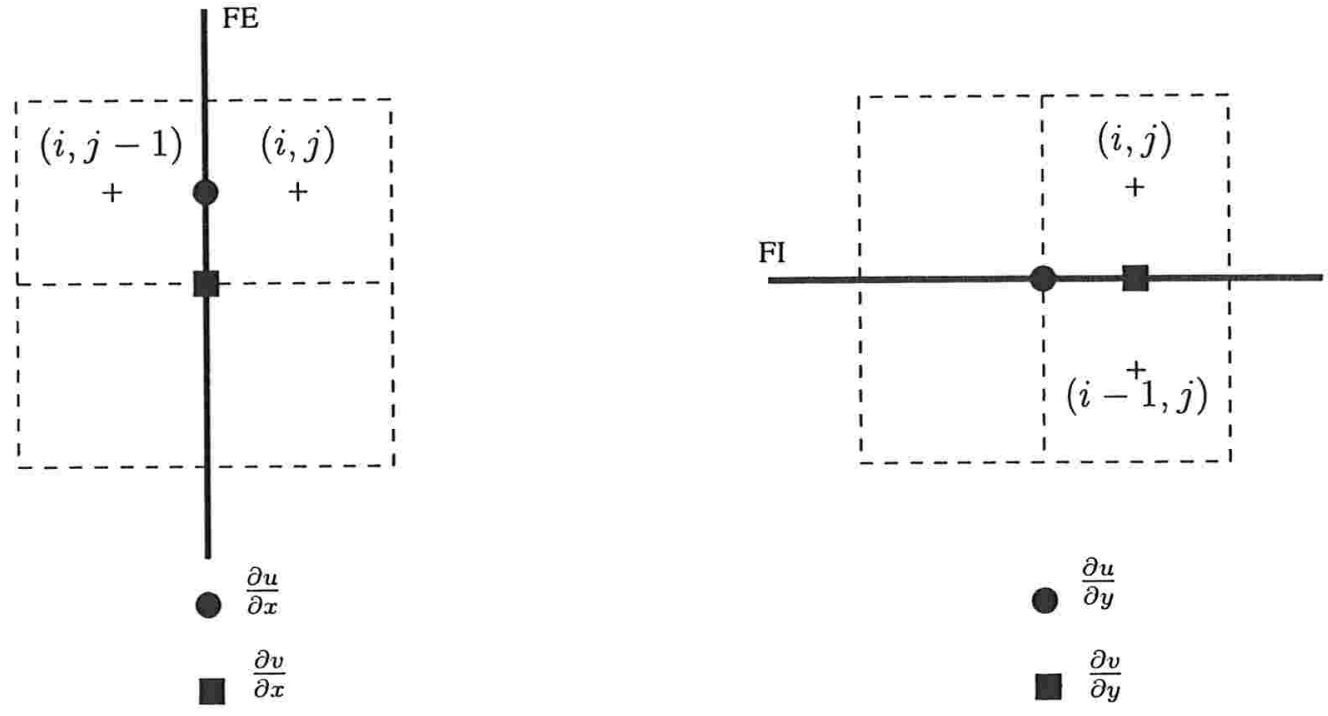

Figura B.3: Pontos de aplicação da condição de contorno para a velocidade em uma malha MAC.

(C6) Notação para a variável $\left(\phi_{l}\right)$ no contorno $\Gamma$

A seguinte notação é usada para a variável $\phi_{l}, l=1,2$ definida no contorno de $\Omega, \Gamma$ :

$$
\left(\phi_{l}\right)_{\Gamma}=\left(\phi_{l}\right)_{\Gamma_{I}} \cup\left(\phi_{l}\right)_{\Gamma_{S}} \cup\left(\phi_{l}\right)_{\Gamma_{E}} \cup\left(\phi_{l}\right)_{\Gamma_{D}},
$$

onde: $\left(\phi_{l}\right)_{\Gamma_{I}},\left(\phi_{l}\right)_{\Gamma_{S}},\left(\phi_{l}\right)_{\Gamma_{E}}$ e $\left(\phi_{l}\right)_{\Gamma_{D}}$ é a variável restrita à fronteira inferior, superior, esquerda e direita do domínio, respectivamente. 


\section{B.2 Condições de contornos numéricas para o passo de projeção}

As seguintes observações das condições de contorno numéricas para as variáveis $\mathbf{u}^{*}$ e $\phi$ são indicadas para dois tipos de condição de contorno da velocidade $\mathbf{u}$ do escoamento:

1. Se $\mathbf{C C}(\mathbf{u})=\mathbf{u}_{b}$ (Dirichlet em $\left.\Gamma\right)$, então pode-se adotar:

- $\mathrm{CC}\left(\mathbf{u}^{*}\right)=\mathrm{u}_{b}$

- $\mathbf{C C}(\phi)=\frac{\partial \phi}{\partial \mathbf{n}}=0$

com $\mathbf{u}=\mathbf{u}^{*}-\Delta t \nabla \phi$ nos pontos internos e fronteira.

2. Se $\mathbf{C C}(\mathbf{u})=\frac{\partial \mathbf{u}}{\partial \mathbf{n}}=\left(h_{u}, h_{v}\right)($ Neumann em $\Gamma)$, então pode-se adotar:

- $\mathbf{C C}\left(\mathbf{u}^{*}\right)=\frac{\partial \mathbf{u}^{*}}{\partial \mathbf{n}}=\left(h_{u}, h_{v}\right)$

- $\mathrm{CC}(\phi)=\phi_{\Gamma}=0$

com $\mathbf{u}=\mathbf{u}^{*}-\Delta t \nabla \phi$ aplicado nos pontos internos, fronteiras e fantasmas.

A prova da ordem de aproximação obtida usando-se tais condições de contornos numéricas é dada na Seção B.3.

No caso de se ter condição de contorno mistas: Dirichlet e Neumann, deve adotarse para as variáveis auxliares a respectiva condição de contorno associada à condição de contorno da velocidade dada acima.

\section{B.3 Ordem de aproximação da velocidade no con- torno}

Nesta Seção são apresentadas alguns lemas que permitem estabelecer a ordem da aproximação no contorno da velocidade usando-se o RKE2 e as condições de contorno numéricas para as variáveis auxiliares dadas na seção anterior.

A seguir os indices $(i, j)$ correspondem aos indices associados à uma célula fronteira e a variável $\phi$ designa qualquer uma das variáveis $\phi_{1}$ ou $\phi_{2}$ associados à váriavel auxiliar no passo de projeção do RKE2. 
Lema 1. Seja $\phi$ uma função escalar definida em $\Omega=[a, b] \times[c, d]$. Se:

(a) $\phi_{\Gamma}=0$.

(b) As aproximações centradas de segunda ordem são aplicadas para a primeira e segunda derivada.

(c) Os indices (i,j) denotam uma célula fronteira,

então,

1. $E m \Gamma_{E}$ :

$$
\begin{aligned}
{\frac{\partial^{2} \phi}{\partial x^{2}}}_{i, j-1 / 2} & \approx O\left(\Delta x^{2}\right) \\
{\frac{\partial \phi}{\partial y_{i-1 / 2, j}}} & \approx-\frac{\partial \phi}{\partial y_{i-1 / 2, j-1}}+O\left(\Delta y^{2}\right)
\end{aligned}
$$

2. $E m \Gamma_{I}$ :

$$
\begin{aligned}
{\frac{\partial^{2} \phi}{\partial y^{2}}}_{i-1 / 2, j} & \approx O\left(\Delta y^{2}\right), \\
{\frac{\partial \phi}{\partial x_{i, j-1 / 2}}} & \approx-\frac{\partial \phi}{\partial x_{i-1, j-1 / 2}}+O\left(\Delta x^{2}\right) .
\end{aligned}
$$

Expressões semelhantes as dadas em (B.10),(B.11), (B.12) e (B.13) são válidas em $\Gamma_{D}$ e $\Gamma_{S}$, respectivamente.

Prova.

1. Seja $(i, j)$ índices de uma célula fronteira esquerda. Segue da hipótse (a) do Lema 1:

$$
\begin{aligned}
\phi_{i, j-1 / 2} & =0, \\
\phi_{i, j} & \approx-\phi_{i, j-1}, \\
\phi_{i-1, j} & \approx-\phi_{i-1, j-1} .
\end{aligned}
$$

Pela condição (b), a segunda e primeira derivada são aproximadas por:

$$
\begin{aligned}
{\frac{\partial^{2} \phi}{\partial x^{2}}}_{i, j-1 / 2} & =\frac{\phi_{i, j}-2 \phi_{i, j-1 / 2}+\phi_{i, j-1}}{2\left(\frac{\Delta x}{2}\right)^{2}}+O\left(\Delta x^{2}\right) . \\
\frac{\partial \phi}{\partial y}{ }_{i-1 / 2, j} & =\frac{\phi_{i, j}-\phi_{i-1, j}}{\Delta y}+O\left(\Delta y^{2}\right) .
\end{aligned}
$$


Substituindo-se as relações (B.14) e (B.15) em (B.17) obtem-se a equação (B.10). Enquanto que as relações (B.15) e (B.16) em (B.18) determinam a equação (B.11).

2. Seja (i,j) uma célula fronteira inferior, a condição (a) implica que:

$$
\begin{aligned}
\phi_{i-1 / 2, j} & =0 \\
\phi_{i, j} & \approx-\phi_{i-1, j}, \\
\phi_{i, j-1} & \approx-\phi_{i-1, j-1} .
\end{aligned}
$$

Devido à aproximação das derivadas por diferenças centradas de segunda ordem (condição (b)), tem-se:

$$
\begin{aligned}
{\frac{\partial^{2} \phi}{\partial y^{2}}}_{i-1 / 2, j} & =\frac{\phi_{i, j}-2 \phi_{i-1 / 2, j}+\phi_{i-1, j}}{2\left(\frac{\Delta y}{2}\right)^{2}}+O\left(\Delta y^{2}\right), \\
\frac{\partial \phi}{\partial x}_{i, j-1 / 2} & =\frac{\phi_{i, j}-\phi_{i, j-1}}{\Delta x}+O\left(\Delta x^{2}\right) .
\end{aligned}
$$

Usando-se a relação (B.19) e (B.20) em (B.22) obtem-se a equação (B.12) e usando-se as relações (B.20) e (B.21) determina-se (B.13).

Lema 2. Sejam $\phi_{l}, l=1,2$ a variável escalar associada ao estágio $l$ do passo de projeção do método explícito de Runge-Kutta de segunda ordem. Se a condição (B.9) é satisfeita para pontos internos do dominio, as seguintes relações são verdadeiras:

$$
\begin{aligned}
& \frac{\partial \phi_{l}}{\partial x} \approx O\left(\Delta t^{l}\right)+O\left(\Delta x^{2}\right) \\
& \frac{\partial \phi_{l}}{\partial y} \approx O\left(\Delta t^{l}\right)+O\left(\Delta x^{2}\right) .
\end{aligned}
$$

Prova.

A demonstração é feita para o primeiro estágio do método de Runge-Kutta explícito. Um processo similar emprega-se para demonstrar o segundo estágio.

Somando as componentes correspondentes das equações (B.1) e (B.3), pode-se obter as seguintes relações:

$$
\begin{aligned}
& \frac{u^{n+1 / 2}-u^{n}}{\Delta t / 2}+(\mathbf{u} \cdot \nabla \mathbf{u})_{x}^{n}=-\left(\frac{\partial q_{1}^{n}}{\partial x}+\frac{\partial \phi_{1}^{n}}{\partial x}\right)+\nabla \cdot[2 \mu \mathrm{d}]_{x}^{n}+f_{x}^{n} \\
& \frac{v^{n+1 / 2}-v^{n}}{\Delta t / 2}+(\mathbf{u} \cdot \nabla \mathbf{u})_{y}^{n}=-\left(\frac{\partial q_{1}^{n}}{\partial y}+\frac{\partial \phi_{1}^{n}}{\partial y}\right)+\nabla \cdot[2 \mu \mathrm{d}]_{y}^{n}+f_{y}^{n}
\end{aligned}
$$


As relações (B.26) e (B.27) devem aproximar as seguintes igualdades:

$$
\begin{aligned}
& \frac{u^{n+1 / 2}-u^{n}}{\Delta t / 2}+O(\Delta t)+(\mathbf{u} \cdot \nabla \mathbf{u})_{x}^{n}=-\frac{\partial p^{n}}{\partial x}+\nabla \cdot[2 \mu \mathbf{d}]_{x}^{n}+f_{x}^{n} \\
& \frac{v^{n+1 / 2}-v^{n}}{\Delta t / 2}+O(\Delta t)+(\mathbf{u} \cdot \nabla \mathbf{u})_{y}^{n}=-\frac{\partial p^{n}}{\partial y}+\nabla \cdot[2 \mu \mathbf{d}]_{y}^{n}+f_{y}^{n} .
\end{aligned}
$$

Comparando-se as equações (B.26) e (B.28) e as equações (B.27) e (B.29), obtem-se:

$$
\begin{aligned}
& \frac{\partial \phi_{1}^{n}}{\partial x}=\frac{\partial p^{n}}{\partial x}-\frac{\partial q_{1}^{n}}{\partial x}+O(\Delta t), \\
& \frac{\partial \phi_{1}^{n}}{\partial y}=\frac{\partial p^{n}}{\partial y}-\frac{\partial q_{1}^{n}}{\partial y}+O(\Delta t) .
\end{aligned}
$$

Devido à consideração (C3) entre $p$ e $q_{l}$ (equações (B.9)), temos que:

$$
\begin{aligned}
& \frac{\partial \phi_{1}^{n}}{\partial x}=O(\Delta t)+O\left(\Delta x^{2}\right) \\
& \frac{\partial \phi_{1}^{n}}{\partial y}=O(\Delta t)+O\left(\Delta y^{2}\right) .
\end{aligned}
$$

No segundo estágio, seguindo um raciocínio semelhante, e o fato que o método é de segunda ordem no tempo, tem-se que:

$$
\begin{aligned}
& \frac{\partial \phi_{2}^{n}}{\partial x}=O\left(\Delta t^{2}\right)+O\left(\Delta x^{2}\right), \\
& \frac{\partial \phi_{2}^{n}}{\partial y}=O\left(\Delta t^{2}\right)+O\left(\Delta y^{2}\right) .
\end{aligned}
$$

De todo o anterior seguem (B.24) e (B.25).

A fim de simplificar as notações a seguir, as seguintes lemas assumem que a densidade do fluido $\rho$ é 1 .

\section{Lema 3. (Caso: Condição de contorno Dirichlet para a velocidade)}

Seja u o campo de velocidade do fluido com condições de contorno do tipo Dirichlet:

$$
\mathbf{u}_{\Gamma}=\mathbf{u}_{b}
$$

obtido pelo método projeção explícita de segunda ordem dada na Seção 4.2.2. Se cumprem as seguintes condições:

1. $\mathbf{u}^{*}$ é calculada como indicada na consideração (C2) (Seção B.1.1) 
2. $\phi$ é calculada da equação

$$
\begin{aligned}
\nabla^{2} \phi & =\frac{\nabla \cdot \mathrm{u}^{*}}{\Delta t} \\
\frac{\partial \phi}{\partial \vec{n}} & =0 .
\end{aligned}
$$

3. $\mathbf{u}$ é calculada via o passo de projeção: $\mathbf{u}=\mathbf{u}^{*}-\Delta t \nabla \phi$ em todos os pontos internos e fronteira;

4. u nos pontos fantasmas é calculada via a condição de contorno dada para ela;

então:

(i) $\nabla \cdot \mathbf{u}=O\left(\Delta x^{2}\right)+O\left(\Delta y^{2}\right)$,

(ii) $\left.\mathbf{u}\right|_{\Gamma}=\mathbf{u}_{b}$

Lema 4. (Caso: Condição de contorno do tipo Neumann para a velocidade)

Seja u o campo de velocidade do fluido com condições de contorno do tipo Neumann:

$$
\frac{\partial \mathbf{u}}{\partial \mathbf{n}}=\mathbf{h}_{b}=\left(h_{u}, h_{v}\right),
$$

obtido mediante o método de projeção explícito de segunda ordem dada na Seção 4.2.2. Se cumprem as seguintes condições:

1. $\mathbf{u}^{*}$ é calculada como indicada na consideração (C2) ( Seção B.1.1);

2. $\phi$ é calculada da equação:

$$
\begin{aligned}
\nabla^{2} \phi & =\frac{\nabla \cdot \mathbf{u}^{*}}{\Delta t} \\
\left.\phi\right|_{\Gamma} & =0 .
\end{aligned}
$$

3. $\mathbf{u}$ é calculada via o passo de projeção: $\mathbf{u}=\mathbf{u}^{*}-\Delta t \nabla \phi$ em todos os pontos internos, fronteira e fantasmas

então:

(i) nas células internas: $\nabla \cdot \mathbf{u}=O\left(\Delta x^{2}\right)+O\left(\Delta y^{2}\right)$, 
(ii) no lado esquerdo do contorno, $\Gamma$

$$
\begin{aligned}
\left.\frac{\partial u}{\partial x}\right|_{i, j-1 / 2} & =h_{u}+O\left(\Delta x^{4}\right), \\
\left.\frac{\partial v}{\partial x}\right|_{i-1 / 2, j-1 / 2} & =h_{v}+O\left(\Delta x^{3}\right),
\end{aligned}
$$

onde (i.j) corresponde a uma célula interna adjacente á fronteira esquerda;

(iii) no lado inferior de $\Gamma$

$$
\begin{aligned}
\left.\frac{\partial u}{\partial y}\right|_{i-1 / 2, j-1 / 2} & =h_{u}+O\left(\Delta y^{3}\right) \\
\left.\frac{\partial v}{\partial y}\right|_{i-1 / 2, j} & =h_{v}+O\left(\Delta y^{4}\right)
\end{aligned}
$$

onde $(i, j)$ coreesponde a uma célula interna adjacente à fronteira inferior.

Expressões semelhantes a (B.40) e (B.41) são válidas na fronteira direita, da mesma forma no lado superior são válidas relações semelhantes a(B.42) e (B.43).

A prova do Lema 3 é direta. Uma prova do Lema 4 é apresentada a seguir.

\section{Prova do Lema 4}

(i) Seja (m.n) índices de uma célula internas e/ou fronteira. Aplicando-se aproximações centradas de segunda ordem para o divergente e considerando-se as hipóteses 2 e 3 do lema 4, obtem-se que:

$$
\nabla \cdot \mathbf{u}_{m, n}=O\left(\Delta x^{2}\right)+O\left(\Delta y^{2}\right)
$$

(ii) A hipótese 1 do lema 4 expressa que o cálculo de $\mathbf{u}^{*}$ nos pontos fronteira ou fantasmas é feito através da seguinte relação:

$$
\frac{\partial \mathbf{u}^{*}}{\partial \mathbf{n}}=\left(h_{u}, h_{v}\right)
$$

onde $\frac{\partial \mathbf{u}^{*}}{\partial \mathbf{n}}$ é aproximada através de diferenças centradas ou não centradas de segunda ordem, segundo seja cada caso.

A demosntração sera feita para o contorno esquerdo do domínio. Um similar raciocinio pode ser aplicado para os outros contornos.

Seja $(i, j)$ índices de uma célula fronteira esquerda, então: 
E1 $(i, j-1 / 2)$ é um ponto fronteira para a variável, $u$. Para tal ponto é válida a hipótese 3 do 4 Logo,

$$
\left.\frac{\partial u}{\partial x}\right|_{i, j-1 / 2}=\frac{\partial u^{*}-\Delta t \frac{\partial \phi}{\partial x}}{\partial x}=\frac{\partial u^{*}}{\partial x}-\Delta t \frac{\partial^{2} \phi}{\partial x^{2}}
$$

Usando-se a hipótese 1. e a relação (B.10) e a condição $\Delta t=O\left(\Delta x^{2}\right)$, tem-se que:

$$
\left.\frac{\partial u}{\partial x}\right|_{i, j-1 / 2}=h_{u}-\Delta t O\left(\Delta x^{2}\right)=h_{u}+O\left(\Delta x^{4}\right) .
$$

E2 (i-1/2, j-1/2) não é nenhum ponto definido na consideração (C5). Para este ponto aproximações centradas de segunda ordem são usadas. Logo,

$$
\left.\frac{\partial v}{\partial x}\right|_{i-1 / 2, j-1 / 2}=\frac{v_{i-1 / 2, j}-v_{i-1 / 2, j-1}}{\Delta x} .
$$

Devido à hipótese (3), tem-se:

$$
\begin{aligned}
\left.\frac{\partial v}{\partial x}\right|_{i-1 / 2, j-1 / 2} & =\frac{\left(v_{i-1 / 2, j}^{*}-\left.\Delta t \frac{\partial \phi}{\partial y}\right|_{i-1 / 2, j}\right)-\left(v_{i-1 / 2, j-1}^{*}-\left.\Delta t \frac{\partial \phi}{\partial y}\right|_{i-1 / 2, j-1}\right)}{\Delta x} \\
& =\frac{v_{i-1 / 2, j}^{*}-v_{i-1 / 2 . j-1}^{*}}{\Delta x}-\frac{\Delta t}{\Delta x}\left(\left.\frac{\partial \phi}{\partial y}\right|_{i-1 / 2, j}-\left.\frac{\partial \phi}{\partial y}\right|_{i-1 / 2, j-1}\right)
\end{aligned}
$$

Usando-se a relação (B.11), a equação (B.47) pode ser reescrita como:

$$
\left.\frac{\partial v}{\partial x}\right|_{i-1 / 2, j-1 / 2}=\left.\frac{\partial v^{*}}{\partial x}\right|_{i-1 / 2, j-1 / 2}-\frac{\Delta t}{\Delta x}\left(\left.2 \frac{\partial \phi}{\partial y}\right|_{i-1 / 2, j}\right)
$$

Usando-se a equação (B.24) na equação acima, tem-se que:

$$
\begin{aligned}
\left.\frac{\partial v}{\partial x}\right|_{i-1 / 2, j-1 / 2} & =h_{v}+O\left(\frac{\Delta t^{l+1}}{\Delta x}\right)+O\left(\frac{\Delta t^{l} \Delta x^{2}}{\Delta x}\right)+O\left(\frac{\Delta t^{l} \Delta y^{2}}{\Delta x}\right) \\
& =h_{v}+O\left(\Delta x^{3}\right)
\end{aligned}
$$

onde a última relação é devido à consideração 1 , isto é, $\Delta t=O\left(\Delta x^{2}\right)$.

A prova das outras relações são similares à demonstrada. 


\section{Referências Bibliográficas}

[1] Arruda, J.M. Modelagem Matemática de Escoamentos Internos Forçados Utilizando o Método da Fronteira Imersa e o Modelo Físico Virtual. $\mathrm{PhD}$ thesis, Universidade Federal de Uberlândia, 2004.

[2] Balaras, E. Modeling Complex Boundaries Using an External Force Field on Fixed Cartesian Grids in Large-Eddy Simulations. Computers and Fluids. 33 (2004), no. $3,375-404$.

[3] Bell, J.B.; Colella, P.; Glaz, H.M. A Second Order Projection Method for the Incompressible Navier-Stokes Equations. Jornal of Computational Physics. 85 (1989), 257-283.

[4] Brown, D.L.; Cortez, R.; Minion, M.L. Accurate Projection Methods for the Incompressible Navier-Stokes Equations. Jornal of Computational Physics. 168 (2001), no. $4,464-499$.

[5] Cezar, H. N. Simulação numérica de escoamentos com superfícies livres e obstáculos em movimento. Master's thesis, Instituto de Ciências Matemáticas e de Computação, Universidade de São Paulo, 2003.

[6] Chorin, A.J. Numerical Solution of the Navier-Stokes Equations. Mathematics of Computation. 22 (1968), no. 104, 745-762.

[7] Chorin, A.J.; Marsden, J.E. A Mathematical Introduction to Fluid Mechanics. Springer-Verlag, 1990. Second Edition. 
[8] Enriquez, S.A. Introdução de Fontes e de Sumidouros em Escoamentos Incompressiveis Bidimensionais por Intermédio do Método da Fronteira Imersa. Master's thesis, Instituto de Matemática e Estatística, Universidade de São Paulo, 2000.

[9] Enriquez-Remigio, S.A.; Roma. Incompressible flows in elastic domains: an immersed boundary method approach. Applied Mathematical Modelling, 29, (2005), 35-54.

[10] Fadlun, E.A.; Verzicco, R.; Orlandi, P.; Mohd-Yusof, J. Combined ImmersedBoundary Finite-Difference Methods for Three-Dimensional Complex Flow Simulations J. Comput. Phys. 161 (2000), 35-60.

[11] Fauci, L.; Peskin, C.S. A Computational Model of Aquatic Animal Locomotion. Journal of Computational Physics. 77 (1988), no. 1, 85-108.

[12] Fernandez-Feria, R.; Sanmiguel-Rojas, E. An Explicit Projection Method for Solving Incompressible Flows Driven by a Pressure Difference. Computers \& Fluids. (2004), 463-483.

[13] Glowinski, R.; Pan, T.W.; Hesla, T.I.; Joseph, D.D. A Distributed Lagrange Multiplier/Fictitious Domain Method for Particulate Flows. International Journal of Multiphase Flow. 25 (1999), no. 5, 755-794.

[14] Goldstein, D.; Handler, R.; Sirovich, L. Modeling a No-Slip Flow Boundary with an External Force Field. Journal of Computational Physics. 105 (1993), 354-366.

[15] Harlow, F.H.; Welch, J.E. Numerical Calculation of Time-Dependent Viscous of Incompressible Flow of Fluid with Free Surface. Physics Fluids. 8 (1965), 2182-2189.

[16] Hu, H.H. Direct Simulation of Flows of Solid-Liquid Mixture. Int. J. Multiphase Flow. 22 (1996), no. 2, 335-352.

[17] Hu, H.H.; Joseph, D.D.; Crochet, M.J. Direct Simulation of Fluid Particle Motions. Theor. Comput. Fluid Dyn. 3 (1992), 285-306.

[18] Iaccarino, G.; Kalitzin, G.; Elkins, CJ. Numerical and experimental investigation of the turbulent flow in ribbed serpentine passage, Annual Research Briefs. Center for Turbulence Research, 2003, 379-88.

[19] Joseph, D.D. Interrogations of Direct Numerical Simulation of Solid-Liquid Flow. http://www.efluids.com/efluids/books/joseph.htm. 
[20] Juárez, L.H.; Glowinski, R. Numerical Simulation of The Motion of Pendula in an Incompressible Viscous Fluid by Lagrange Multipler/Fictitious Domain Methods. Fourteenth International Conference on Domain Decomposition Methods.

[21] Karrniadakis, G.E.; Israeli, M.; Orszag, S.A. High-Order Splitting Methods for the Incompressible Navier-Stokes Equations. Journal of Computational Physics. 97 (1991), no. $12,414-443$.

[22] Kim, J.; Kim, D.; Choi, H. An Immersed-Boundary Finite-Volume Method for Simulations of Flow in Complex Geometries. Journal of Computational Physics. 171 (2001), no. 1, 132-150.

[23] Kim, J.; Moin, P. Application of a Fractional-Step Method to Incompressible NavierStokes Equations. Journal of Computational Physics. 59 (1985), 308-323.

[24] Langtangen, H.P.; Kent-Andre, M.; Winther, R. Numerical Methods for Incompressible Viscous Flow. Advances in Water Resources. 25 (2002), no. 11, 1125-1146.

[25] Lima, L.F.; Silva, E.; Silveira, A.; Damasceno, J.J.R. Numerical Simulation of TwoDimensional Flows Over a Circular Cylinder Using the Immersed Boundary Method. Journal of Computational Physics. 189 (2002), no. 2, 351-370.

[26] Majumdar, S.; Iaccarino, G.; Durbin, P. RANS Solvers with Adaptive Structured Boundary Non-Conforming Grids. Center for Turbulence Research Annual Research Briefs 2001. (2001), 353-366.

[27] McQueen, D.M.; Peskin, C.S. Heart Simulation by an Immersed Boundary Method with Formal Second-Order Accuracy and Reduced Numerical Viscosity. 2000.

[28] Ming-Chih L. Simulation of the flow Past an Array of Circular Cylinders as a Test of the Immersed Boundary Method; Ph.D. thesis, New York University, 1998.

[29] Mittal, R; Seshadri, V.; Udaykumar HS, Flutter, tumble and vortex induced autorotation, Theoretical and Computational Fluid Dynamics, 17 (2004), no. 3, 165-70.

[30] Mohd-Yusof, J. Combined Immersed-Boundary/B-Spline Methods for Simulations of Flow in Complex Geometries. Annual Research Briefs. Center for Turbulence Research, NASA Ames and Stanford University. 1997.

[31] Oliveira, A. Técnicas Computacionais para Dinâmica dos Fluidos : Conceitos Básicos e Aplicacões. Edusp, 2000. 
[32] Oliveira, J.E.S.; Lima e Silva, A.F.L.; Guimarães, G.; Silveira-Neto, A. Simulação Numérica do Escoamento a Baixo Reynolds Sobre um Cilindro de Diâmetro Variável usando MFI / MFV, Proceedings of the $10^{\circ}$ Brazilian Congress of Thermal Sciences and Engineering, 2004.

[33] Padilla, E.L.M. Simulação numérica de Grandes Escalas com Modelagem Dinâmica, Aplicada à Convecção Mista. Master's thesis, Universidade Federal de Uberlândia, 2000.

[34] Peskin, C.S. Flow Patterns Around Heart Valves: A Numerical Method. Journal of Computational Physics. 10 (1972), 252-271.

[35] Peski, C.S. The immersed bounday method. Acta Numerica. 1-39

[36] Peskin, C.S.; McQueen, D.M. A Three-Dimensional Computational Method for Blood Flow in the Heart. I. Immersed Elastic Fibers in a Viscous Incompressible Fluid. Journal of Computational Physics. 81 (1989), no. 2, 372-405.

[37] Roma, A.M. A Multilevel self Adaptive Version of the Immersed Boundary Method; Ph.D. thesis, New York University, 1994.

[38] Rosar, M.E. A three-Dimensional Computer Model for Fluid Flow Through a Collapsible Tube; Ph.D. thesis, Courant Institute of Mathematical Sciences-New York University, 1994.

[39] Saiki, E.M. Biringen, S. Numerical Simulation of a Cylinder in Uniform Flow: Application of a Virtual Boundary Method. Journal of Computational Physics. 123 (1996), 450-465.

[40] Swarztrauber, P. Sweet, R. Efficient FORTRAN subprograms for the solution of elliptic partial differential equations. "Technical Report TN/IA-109" , NCAR, 1995.

[41] Silva, A. R.; Carvalho, G. B.; Lima e Silva, A.F.L.; Mansur, J.; Silveira-Neto, A. Modelagem matemática e simulação numérica de escoamentos sobre corpos móveis utilizando-se o método da fronteira imersa. In Submitted to Proceedings of the $10^{\circ}$ Brazilian Congress of Thermal Sciences and Engineering, 2004.

[42] Silveira-Neto, A. Introdução Turbulência dos Fluidos, Apostila do Curso de PósGraduaçãoi em Eng. Mecânica. LTCM/FEMEC, Universidade de Uberlândia. 
[43] Sousa, F.S.; Mangiavacchi, N.; Nonato, L.G.; Castelo, A.; Tomé, M.F. Ferreira, V.G.; Cuminato, J.A.; McKee, M. A Front-Tracking/Front-Capturing Method for the Simulation of 3D Multi-Fluid Flows with Free Surfaces. Journal of Computational Physics. 198 (2004), 469-499.

[44] Uhlmann, M. First Experiments with the Simulation of Particulate Flows. Technical Report no. 1020, CIEMAT. Madrid-Spain, 2003.

[45] Unverdi, S.O.; Tryggavason, G. A Front-Trcking Method for Viscous Incompressible Multi-Fluid Flows. Journal of Computational Physics, 100 (1992), 25-60.

[46] Van-Kan, J. A Second-Order Accurate Pressure-Correction Scheme for Viscous Incompressible Flow. SIAM Journal on Scientific and Statistical Computation. 7 (1986), 870-891.

[47] Verzicco, J.; Iaccarino, M.; Fatica, M.; Orlandi, P Flow in an impeller Stirred Tank using an Immersed Boundary Method. Annual Research Briefs. Center for Turbulence Research, 2000, 251-261.

[48] Verzicco, R.; Fatica, M.; Iaccarino, G.; Moin, P.; Khalighi, B. Large Eddy Simulation of a Road Vehicle with Drag Reduction Devices, AIAA Journal, 4 2002, no. 12, 24472455.

[49] Vilaça, A. C.; Oliveira, J.E.S.; Lima e Silva, A.F.L.; Silveira-Neto, A. Modelagem Matemática e Simulação Numérica do escoamento sobre uma partícula em queda livre, XXXI Congresso de Sistemas Particulados ENEMP, Uberlândia-MG-Brasil, 2004.

[50] Weinan, E.; Jian-Guo, L. Vorticity Boundary Condition and Related Issues for Finite Difference Schemes. Journal of Computational Physics. 124 (1996), 368-382.

[51] Ye, T.; Mittal, R.; Udaykumar, H.S.; Shyy, W. An Accurate Cartesian Grid Method for Viscous Incompressible Flows with Complex Immersed Boundaries. Journal of Computational Physics. 156 (1999), 209-240.

[52] Yu-Heng, T.; Ferziger, J.H. A Ghost-Cell Immersed Boundary Method for Flow in Complex Geometry. Journal of Computational Physics. 192 (2003), 593-623. 